Roughing up Beta: Continuous vs. Discontinuous Betas, and the Cross-Section of Expected Stock Returns

\author{
Tim Bollerslev, Sophia Zhengzi Li and Viktor Todorov
}

CREATES Research Paper 2014-48 


\title{
Roughing up Beta: Continuous vs. Discontinuous Betas, and the Cross-Section of Expected Stock Returns*
}

\author{
Tim Bollerslev ${ }^{\dagger}$ Sophia Zhengzi Li $\stackrel{\ddagger}{\ddagger}$ and Viktor Todorov ${ }^{\S}$ \\ First Version: November 14, 2013 \\ This Version: December 4, 2014
}

\begin{abstract}
Motivated by the implications from a stylized equilibrium pricing framework, we investigate empirically how individual equity prices respond to continuous, or "smooth," and jumpy, or "rough," market price moves, and how these different market price risks, or betas, are priced in the cross-section of expected returns. Based on a novel highfrequency dataset of almost one-thousand individual stocks over two decades, we find that the two rough betas associated with intraday discontinuous and overnight returns entail significant risk premiums, while the intraday continuous beta is not priced in the cross-section. An investment strategy that goes long stocks with high jump betas and short stocks with low jump betas produces significant average excess returns. These higher risk premiums for the discontinuous and overnight market betas remain significant after controlling for a long list of other firm characteristics and explanatory variables previously associated with the cross-section of expected stock returns.
\end{abstract}

JEL classification: C13, C14, G11, G12

Keywords: Market price risks; jump betas; high-frequency data; cross-sectional return variation.

${ }^{*}$ An earlier version of the paper by the first two authors was circulated under the different title: "Roughing up the CAPM: Jump Betas and the Cross-Section of Expected Stock Returns." We would like to thank the Editor (Ken Singleton) and three anonymous referees for their detailed and thoughtful comments. We would also like to thank Turan Bali, Jia Li, Andrew Patton, Mark Schroder, and George Tauchen, along with seminar participants at numerous universities, financial institutions, and different conferences for their many helpful comments and suggestions. The research was partly funded by an NSF grant to the NBER. Bollerslev also acknowledges support from CREATES funded by the Danish National Research Foundation (DNRF78), while Li gratefully acknowledges financial support from the 2012 Morgan Stanley Prize for Excellence in Financial Markets.

${ }^{\dagger}$ Department of Economics, Duke University, Durham, NC 27708, and NBER and CREATES; e-mail: boller@duke.edu.

${ }^{\ddagger}$ Department of Finance, Eli Broad College of Business, Michigan State University, East Lansing, MI 48824; e-mail: lizhengzi@broad.msu.edu.

${ }^{\S}$ Department of Finance, Kellogg School of Management, Northwestern University, Evanston, IL 60208; e-mail: v-todorov@northwestern.edu. 


\section{Introduction}

The idea that only systematic market price risk should be priced represents one of the cornerstones of finance. Even though numerous studies over the past half-century have called into question the ability of the CAPM to fully explain the cross-section of expected stock returns, the beta of an asset arguably remains the most commonly used systematic risk measure in financial practice. ${ }^{1}$ Meanwhile, more recent empirical evidence pertaining to the equity risk premium and the pricing of risk at the aggregate market level suggests that the expected return variation associated with discontinuous price moves, or jumps, is priced higher than the expected continuous price variation. ${ }^{2}$

Set against this background, we propose a general pricing framework involving three separate market betas: a continuous beta reflecting "smooth" intraday comovements with the market, and two "rough" betas associated with intraday price discontinuities, or jumps, during the active part of the trading day, and the overnight close-to-open return, respectively. We find that the two rough betas on average exceed the continuous beta. ${ }^{3}$ Moreover, consistent with the idea that investors view intraday smooth, and easier to hedge price moves quite differently from intraday rough and day-to-day overnight price changes, ${ }^{4}$ we find that the risk premiums associated with the two jump betas are both statistically significant and indistinguishable, while the continuous beta does not appear to be priced in the cross-section.

The theoretical framework motivating our empirical investigations and the separate crosssectional pricing of continuous and discontinuous market price risks is very general, and merely assumes the existence of a generic pricing kernel along the lines of Duffie et al. (2000). Importantly, we make no explicit assumptions about the pricing of other non-market price risks. As such, our setup includes the popular long-run risk model of Bansal and Yaron

\footnotetext{
${ }^{1}$ Early work by Fama et al. (1969) and Blume (1970) generally supports the CAPM. Subsequent prominent empirical studies that call into question the explanatory power of market betas for satisfactorily explaining the cross-section of expected returns include Basu (1977, 1983), Roll (1977), Banz (1981), Stattman (1983), Rosenberg et al. (1985), Bhandari (1988), and Fama and French (1992).

${ }^{2}$ Empirical evidence based on aggregate equity index options in support of this hypothesis includes Pan (2002), Eraker et al. (2003), Bollerslev and Todorov (2011), and Gabaix (2012), among others.

${ }^{3}$ The seminal paper by Merton (1976) hypothesizes that jump risks for individual stocks are likely to be non-systematic. On the other hand, more recent empirical evidence of increased cross-asset correlations for higher (in an absolute sense) returns documented in Ang and Chen (2002), among many others, indirectly suggests non-zero systematic jump risk.

${ }^{4}$ Optimally managing market diffusive and jump price risks require the use of different hedging tools and derivative instruments; see e.g., the theoretical analysis in Liu et al. (2003a,b) as well as the more recent discussion in Aït-Sahalia et al. (2009) related to portfolio allocation in the presence of jumps. The increased availability of short-maturity out-of-the-money options, which provide a particular convenient tool for managing jump tail risk, also directly speaks to the practical importance of separately accounting for these different types of risks.
} 
(2004), the habit persistence model of Campbell and Cochrane (1999), and the rare disaster model of Gabaix (2012), as special cases obtained by further restricting the functional form of the pricing kernel and the set of other priced risk factors.

The statistical theory underlying our estimation of the separate betas builds on recent advances in financial econometrics related to the use of high-frequency intraday data and so-called realized volatilities. Bollerslev and Zhang (2003), Barndorff-Nielsen and Shephard (2004a), and Andersen et al. (2005, 2006), in particular, have previously explored the use of high-frequency data and the asymptotic notion of increasingly finer sampled returns over fixed time intervals for more accurately estimating realized betas. In contrast to these earlier studies, which do not differentiate among different types of market price moves, we rely on the theory originally developed by Todorov and Bollerslev (2010) for explicitly estimating separate continuous and discontinuous betas for the open-to-close active part of the trading day, together with overnight betas for the close-to-open returns. ${ }^{5}$

Our actual empirical investigations are based on a novel high-frequency dataset of all the 985 stocks included in the S\&P 500 index over the 1993-2010 sample period. We begin by estimating the three separate betas as well as a standard CAPM regression-based beta for each of the individual stocks on a rolling one-year basis. Consistent with the basic tenets of the simple CAPM, we find that sorting the stocks in our sample on the basis of their betas, results in a positive return differential between the High- and Low-beta quantile portfolios for all of the four different beta estimates. ${ }^{6}$ However, even though all of the return differentials are quite large numerically, the difference in the monthly returns between the High- and Low-beta portfolios constructed on the basis of the standard CAPM betas is not significantly different from zero at conventional levels. Similarly, sorting by our continuous beta estimates, the monthly long-short excess return for the High- minus Low-beta quantile portfolios is not significantly different from zero. On the other hand, sorting stocks on the basis of their discontinuous and overnight betas, as well as their "relative betas" defined by the difference between either of the two jump betas and the standard beta, results in significantly positive risk-adjusted returns on the High-Low portfolios. This holds true for both equally-weighted and value-weighted portfolios.

These portfolio sorts based on returns and betas estimated over the same holding period

\footnotetext{
${ }^{5}$ Branch and Ma (2012), Cliff et al. (2008), and Berkman et al. (2012) also document distinctly different return patterns during trading and non-trading hours.

${ }^{6}$ This contrasts with the recent results in Frazzini and Pedersen (2014), which report an almost flat security market line and highly significant positive CAPM alphas for portfolios "betting against beta." Compared to our investigations, which are limited by the availability of reliable high-frequency intraday data and as such "only" involves 985 relatively large company stocks over the past two decades, the results in Frazzini and Pedersen (2014) are based on a much larger sample of more than twenty thousand stocks spanning almost a full century.
} 
represent the essence of the risk-return relationship implied by our theoretical model. However, more importantly from a practical perspective, we show that these same significant contemporaneous return differentials carry over to a predictive setting, in which we compare the subsequent realized monthly returns of the quintile portfolios based on grouping the stocks according to their past rolling one-year beta estimates.

The predictive return differentials associated with the discontinuous and overnight betas remain statistically significant in double portfolio sorts designed to control for a number of other firm characteristics and risk factors previously associated with the cross-section of expected returns, including firm size, book-to-market ratio, momentum, short-term reversal, idiosyncratic volatility, maximum daily return, illiquidity, and various measures of skewness and kurtosis. Standard predictive Fama-MacBeth regressions further corroborate the idea that only rough market risks are priced. In particular, while the estimated risk premiums associated with the intraday discontinuous and overnight betas are both significant after simultaneously controlling for a long list of firm characteristics and other risk factors, the estimated risk premium associated with the continuous beta is not.

Our main empirical findings rely on a relatively coarse 75-minute intraday sampling frequency for the one-year rolling continuous and jump beta estimation, as a way to guard against non-synchronous trading effects and other market microstructure complications that arise at the highest intraday sampling frequency. However, our results remain robust to the use of other sampling frequencies and inference procedures for the estimation of the betas. Similarly, while our main results for the predictive portfolio sorts and cross-sectional regressions are based on a standard one-year estimation and subsequent one-month holding period, respectively, even stronger results hold true for other estimation windows and return holding periods. Also, while some of the jumps that occur at the aggregate market level are naturally associated with news about the economy, our results remain robust to the exclusion of several important macroeconomic news announcement days. ${ }^{7}$

The idea of allowing for time-varying market betas to help explain the cross-section of expected stock returns is related to the large literature on testing conditional versions of the CAPM. ${ }^{8}$ In response to many of these studies, Lewellen and Nagel (2006) have forcefully argued that the temporal variation required in conditional CAPM betas to fully explain

\footnotetext{
${ }^{7}$ Initial studies documenting large changes in high-frequency intraday returns in response to macroeconomic news announcements include Fleming and Remolona (1999) and Andersen et al. (2003, 2007b).

${ }^{8}$ Early contributions to this literature include Ferson et al. (1987), Bollerslev et al. (1988) and Harvey (1989), among others, along with more recent cross-sectionally oriented studies by Jagannathan and Wang (1996) and Lettau and Ludvigson (2001). Bali et al. (2014) have also recently argued that GARCH-based time-varying conditional betas help explain the cross-sectional variation in expected stock returns, while Hedegaard and Hodrick (2013) show how higher frequency overlapping data may be used in more accurately estimating the conditional CAPM.
} 
the widely documented asset pricing anomalies associated with size, value and momentum appear implausibly large. In contrast to this literature, however, our empirical investigations should not be interpreted as a test of the conditional CAPM. Instead, motivated by our general pricing framework, we simply document that market risks with different degrees of "jumpiness," as determined by our high-frequency-based estimates of the time-varying continuous and jump betas, are priced differently, and that these cross-sectional differences in the returns can not be explained by other firm characteristics or commonly used risk factors. In particular, we are not arguing that market risk is the only source of priced risk in the cross-section.

Our work is also related to, but fundamentally different from, several recent studies that have examined how jump risk may help explain the cross-section of expected stock returns. Jiang and Yao (2013) argue that the size premium, the liquidity premium, and to a lesser extent the value premium are all realized in the cross-sectional differences of jump returns. Cremers et al. (2014) show that market expectations of aggregate jump risk implied from options prices are useful for explaining the cross-sectional variation in expected returns, while Yan (2011) documents that expected stock returns are negatively related to average jump sizes. Our work differs from these studies in at least two important dimensions. First, we focus explicitly on systematic jump risk, as measured by the exposure to non-diversifiable marketwide jumps and the two rough betas. Second, our use of high-frequency data to directly identify the intraday jumps and estimate the betas, sets our study apart from other research inferring the jump risk from daily or lower-frequency data.

Our cross-sectional pricing results also complement recent time-series estimates of the equity risk premium reported in Bollerslev and Todorov (2011) and Gabaix (2012), among others, which suggest that a large portion of the aggregate equity premium and the temporal variation therein may be attributable to jump tail risk. In line with these findings for the aggregate market, the two rough betas associated with intraday jumps and day-to-day overnight price changes directly reflect the individual stocks' systematic response to jump risk, and in turn receive the largest compensation in the cross-section. Intuitively, large stock price movements likely provide better signals about true changes in fundamentals and equity valuations than do smaller within-day price fluctuations, which may simply represent "noise" in the price formation process.

The remainder of the paper is organized as follows. Section 2 formally defines the different betas and the theory underlying their separate pricing within a stylized equilibrium-based asset pricing framework. Readers primarily interested in the empirical results may skip this section. The statistical procedures used for estimating the separate betas are discussed in 
Section 3. Section 4 describes the high-frequency data that we use to estimate the betas and the control variables employed in our empirical investigations. Section 5 presents our initial empirical evidence on the relation between the different betas and the cross-sectional return differences based on single contemporaneous portfolio sorts. Section 6 extends the results to a practically relevant predictive setting and also considers various double portfolio sorts. Section 7 discusses the results of corresponding predictive firm-level cross-sectional regressions and estimates of the risk premiums for the different betas. Section 8 presents the results from a series of robustness checks related to the intraday sampling frequency used in the estimation of the betas, possible non-synchronous trading effects, errors-in-variables in the cross-sectional pricing regressions, the length of the beta estimation and return holding periods, conditional alphas, and the influence of specific macroeconomic news announcements. Section 9 concludes.

\section{Continuous and discontinuous market risk pricing}

Our theoretical framework motivating the different betas and the separate pricing of continuous and discontinuous market price risks is extremely general, and merely relies on no-arbitrage and the existence of a pricing kernel. By the same token, we do not provide explicit equilibrium-based expressions for the separate risk premia. Doing so would require additional assumptions beyond the ones necessary for simply separating the continuous and discontinuous market risk premiums and the corresponding market betas.

To set out the notation, let the price of the aggregate market portfolio be denoted by $P_{t}^{(0)}$, with the corresponding logarithmic price denoted by lowercase $p_{t}^{(0)} \equiv \log P_{t}^{(0)}$. We will assume the following general dynamic representation for the instantaneous return on the market,

$$
d p_{t}^{(0)}=\alpha_{t}^{(0)} d t+\sigma_{t} d W_{t}+\int_{\mathbb{R}} x \widetilde{\mu}(d t, d x)
$$

where $W_{t}$ denotes a Brownian motion describing continuous Gaussian, or "smooth," market price shocks with diffusive volatility $\sigma_{t}$, and $\widetilde{\mu}$ is a (compensated) jump counting measure accounting for discontinuous, or "rough," market price moves. ${ }^{9}$ The drift term $\alpha_{t}^{(0)}$ is explicitly related to the pricing of these separate market risks.

We will denote the cross-section of individual stock prices by $P_{t}^{(i)}, i=1, \ldots, n$. In parallel to the representation for the market portfolio above, we will assume that the instantaneous

\footnotetext{
${ }^{9}$ The compensated jump counting measure is formally related to the actual counting measure $\mu$ for the jumps in $P^{(0)}$ by the expression $\widetilde{\mu}(d t, d x) \equiv \mu(d t, d x)-d t \otimes \nu_{t}(d x)$, where $\nu_{t}(d x)$ denotes the (possibly time-varying) intensity of the jumps, thus rendering the $\widetilde{\mu}$ measure a martingale.
} 
logarithmic price process, $p_{t}^{(i)} \equiv \log P_{t}^{(i)}$, for each of the $n$ individual stocks may be expressed as,

$$
d p_{t}^{(i)}=\alpha_{t}^{(i)} d t+\beta_{t}^{(c, i)} \sigma_{t}^{(i)} d W_{t}+\int_{\mathbb{R}} \beta_{t}^{(d, i)} x \widetilde{\mu}(d t, d x)+\widetilde{\sigma}_{t}^{(i)} d W_{t}^{(i)}+\int_{\mathbb{R}} x \widetilde{\mu}^{(i)}(d t, d x),
$$

where the $W_{t}^{(i)}$ Brownian motion is orthogonal to $W_{t}$, but possibly correlated with $W_{t}^{(j)}$ for $i \neq j$, and the $\mu^{(i)}$ jump measure is orthogonal to $\mu$ in the sense that $\mu(\{t\}, \mathbb{R}) \mu^{(i)}\left(\{t\}, \mathbb{R}^{p}\right)=0$ for every $t$, so that $\mu^{(i)}$ only counts firm specific jumps occurring at times when the market does not jump. By explicitly allowing the individual loadings, or betas, associated with the market diffusive and jump risks to be time-varying, this decomposition of the continuous and discontinuous martingale parts of asset $i$ 's return into separate components directly related to their market counterparts and orthogonal components (in a martingale sense) is extremely general. For the diffusive part, in particular, this entails no assumptions and follows merely from the partition of a correlated bivariate Brownian motion into its orthogonal components (see, e.g., Theorem 2.1.2 in Jacod and Protter, 2012). For the discontinuous part, the decomposition implicitly assumes that the relation between the systematic jumps in the asset and the market index, while time-varying, does not depend on the size of the jumps. ${ }^{10}$ This type of restriction is arguably unavoidable. By their very nature, systematic jumps are relatively "rare," and as such it isn't feasible to identify different jump betas for different jump sizes, let alone identify the "small" jumps in the first place. As discussed further below, this assumption also maps directly into the way in which we empirically estimate jump betas for each of the individual stocks based solely on the "large" sized jumps.

To analyze the pricing of continuous and discontinuous market price risks, we follow standard practice in the asset pricing literature and assume the existence of an economywide pricing kernel of the form (see, e.g., Duffie et al., 2000),

$$
M_{t}=e^{-\int_{0}^{t} r_{s} d s} \mathcal{E}\left(-\int_{0}^{t} \lambda_{s} d W_{s}+\int_{0}^{t} \int_{\mathbb{R}}(\kappa(s, x)-1) \widetilde{\mu}(d s, d x)\right) M_{t}^{\prime},
$$

where $r_{t}$ denotes the instantaneous risk-free interest rate, and $\mathcal{E}(\cdot)$ refers to the stochastic exponential. ${ }^{11}$ The càdlàg $\lambda_{t}$ process and the predictable $\kappa(t, x)$ function account for the pricing of diffusive and jump market price risks, respectively. The last term $M_{t}^{\prime}$ encapsulates the pricing of all other (orthogonalized to the market price risks) systematic risk factors. In

\footnotetext{
${ }^{10}$ Formally, let $s$ denote a time when the market jumps, and $\Delta p_{s}^{(0)} \neq 0$. The representation in (2) then implies that $\Delta p_{s}^{(i)} / \Delta p_{s}^{(0)}=\beta_{s}^{(d, i)}$, allowing the jump beta to vary with the time $s$ but not the actual size of the jump.

${ }^{11}$ Formally, for some arbitrary process $Z, \mathcal{E}(Z)$ is defined by the solution to the $\mathrm{SDE}, \frac{d Y}{Y_{-}}=d Z$, with initial condition $Y_{0}=1$.
} 
parallel to the first part of the expression for $M_{t}$, we will assume that this additional part of the pricing kernel takes the form,

$$
M_{t}^{\prime}=\mathcal{E}\left(-\int_{0}^{t} \lambda_{s}^{\prime} d W_{s}^{\prime}+\int_{0}^{t} \int_{\mathbb{R}}\left(\kappa^{\prime}(s, \mathbf{x})-1\right) \widetilde{\mu}^{\prime}(d s, d \mathbf{x})\right),
$$

where the $W_{t}^{\prime}$ Brownian motion is orthogonal to $W_{t}$, and the two jump measures $\mu$ and $\mu^{\prime}$ are orthogonal in the sense that $\mu(\{t\}, \mathbb{R}) \mu^{\prime}\left(\{t\}, \mathbb{R}^{p}\right)=0$ for every $t$, so that the respective jumps never arrive at the exact same instant. The pricing kernel jointly defined by equations (3) and (4) encompasses almost all parametric asset pricing models hitherto analyzed in the literature as special cases.

To help fix ideas, consider the case of a static pure-endowment economy, with i.i.d. consumption growth and a representative agent with Epstein-Zin preferences. In this basic CCAPM setup, the dynamics of the pricing kernel will be driven solely by consumption. Assuming that the market portfolio represents a claim on total consumption, it therefore follows that $M_{t}^{\prime} \equiv 1$, resulting in a pricing kernel that solely depends on the diffusive Gaussian and discontinuous market price shocks. This same analysis continues to hold true for a representative agent with habit persistence as in Campbell and Cochrane (1999), the only difference being that in this situation the prices of the diffusive and jump market risks will be time-varying due to the temporal variation in the degree of risk-aversion of the representative agent. In general, of course, temporal variation in the investment opportunity set, as in the ICAPM of Merton (1973), may induce additional sources of priced risks that cannot be spanned by the market price risks alone. Leading examples of other state variables that might affect the pricing kernel include the conditional mean and volatility of consumption growth as in Bansal and Yaron (2004), and the time-varying probability of a disaster as in Gabaix (2012) and Wachter (2013). ${ }^{12}$ However, given our primary focus on the pricing of market price risk, we purposely do not take a stand on what these other risk factors might be, instead simply relegating their influence to the additional $M_{t}^{\prime}$ part of the pricing kernel.

The pricing kernel in (3) has also been widely used in the literature on derivatives pricing. For reasons of analytical tractability, in that literature it is also commonly assumed that $\lambda_{t}$ is proportional to the market diffusive volatility $\sigma_{t}$, that the jump intensity $\nu_{t}(d x)$ is affine in $\sigma_{t}^{2}$, and that the price of jump risk $\kappa(t, x)$ is time-invariant; see, e.g., Duffie et al. (2000) where it is shown that these assumptions greatly facilitate the calculation of "closed form" derivatives pricing formulas. These same assumptions also imply that the equity risk premium should be proportional to the variance of the aggregate market portfolio. ${ }^{13}$

\footnotetext{
${ }^{12}$ In models involving non-financial wealth, so that the market portfolio and the total wealth portfolio aren't perfect substitutes, additional sources of risks will also naturally arise.

${ }^{13}$ This simple relationship has, of course, been extensively investigated in the empirical asset pricing
} 
In general, it follows readily by a standard change-of-measure (see, e.g., Jacod and Shiryaev, 2002) that without any additional restrictions on the pricing kernel defined by (3) and (4), the instantaneous market risk premium must satisfy,

$$
\alpha_{t}^{(0)}-r_{t}-\delta_{t}^{(0)}-q_{t}^{(0)}=\gamma_{t}^{c}+\gamma_{t}^{d},
$$

where $\delta_{t}^{(0)}$ refers to the dividend yield on the market portfolio, and the compensation for continuous and discontinuous market price risks are determined by,

$$
\gamma_{t}^{c} \equiv \sigma_{t} \lambda_{t}, \quad \text { and } \quad \gamma_{t}^{d} \equiv \int_{\mathbb{R}} x \kappa(t, x) \nu_{t}(d x),
$$

respectively, while $q_{t}^{(0)}$ represents a standard convexity adjustment term. ${ }^{14}$ Since the compensation stemming from $M_{t}^{\prime}$ is orthogonal to the compensation for market price risk, this expression for $\alpha_{t}^{(0)}$ only depends on the first part of the pricing kernel.

For the individual assets, however, even though the $W_{t}^{(i)}$ and $\mu^{(i)}$ diffusive and jump risks are orthogonal to the corresponding market diffusive and jump risk components, they may nevertheless be priced in the cross-section as they could be correlated with the $W_{t}^{\prime}$ and $\mu^{\prime}$ risks that appear in the $M_{t}^{\prime}$ part of the pricing kernel. Denoting the part of the instantaneous risk premium for asset $i$ arising from this separate pricing of $W_{t}^{(i)}$ and $\mu^{(i)}$ by $\widetilde{\alpha}_{t}^{(i)}$, it follows again by standard arguments that,

$$
\alpha_{t}^{(i)}-r_{t}-\delta_{t}^{(i)}-q_{t}^{(i)}=\beta_{t}^{(c, i)} \gamma_{t}^{c}+\beta_{t}^{(d, i)} \gamma_{t}^{d}+\widetilde{\alpha}_{t}^{(i)},
$$

where $\delta_{t}^{(i)}$ refers to the dividend yield of asset $i$, and $q_{t}^{(i)}$ denotes a standard convexity adjustment term stemming from the pricing of market price risks. ${ }^{15}$

If $\widetilde{\alpha}_{t}^{(i)} \equiv 0$, as would be implied by $M_{t}^{\prime} \equiv 1$, and if $\beta_{t}^{(c, i)}$ and $\beta_{t}^{(d, i)}$ were also the same, the expression in (6) trivially reduces to a simple continuous-time one-factor CAPM that linearly relates the instantaneous return on stock $i$ to its single beta. The restriction that $\beta_{t}^{(c, i)}=\beta_{t}^{(d, i)}$ implies that the asset responds the same to market diffusive and jump price increments, or intuitively that the asset and the market co-move the same during "normal" times and periods of "extreme" market moves. If, on the other hand, $\beta_{t}^{(c, i)}$ and $\beta_{t}^{(d, i)}$ differ, empirical evidence for which is provided below, the cross-sectional variation in the continuous and jump betas may be used to identify their separate pricing. Importantly, this remains true in the presence of other priced risk factors, when $\widetilde{\alpha}_{t}^{(i)}$ is not necessarily equal to zero.

literature; see, e.g., Bollerslev et al. (2012) and the many additional references therein.

${ }^{14}$ The $q_{t}^{(0)}$ term is formally given by $\frac{1}{2} \sigma_{t}^{2}+\int_{\mathbb{R}}\left(e^{x}-1-x\right) \nu_{t}(d x)$.

${ }^{15}$ In parallel to the expression for $q_{t}^{(0)}$ above, $q_{t}^{(i)}=\frac{1}{2}\left(\beta_{t}^{(c, i)} \sigma_{t}\right)^{2}+\int_{\mathbb{R}}\left(e^{\beta_{t}^{(d, i)} x}-1-\beta_{t}^{(d, i)} x\right) \nu_{t}(d x)$. 
In practice, of course, the returns on the assets will have to be measured over some nontrivial time-interval, say $h>0$. Let $r_{t, t+h}^{(i)} \equiv p_{t+h}^{(i)}-p_{t}^{(i)}$ denote the corresponding logarithmic return on asset $i$. For empirical tractability assume that the betas remain constant over that same (short) time-interval. The integrated conditional risk premium for asset $i$ may then be expressed as,

$$
\begin{aligned}
\mathbb{E}_{t}\left(r_{t, t+h}^{(i)}-\int_{t}^{t+h}\left(r_{s}+\delta_{s}^{(i)}+q_{s}^{(i)}\right) d s\right)= & \beta_{t}^{(c, i)} \mathbb{E}_{t}\left(\int_{t}^{t+h} \gamma_{s}^{c} d s\right)+\beta_{t}^{(d, i)} \mathbb{E}_{t}\left(\int_{t}^{t+h} \gamma_{s}^{d} d s\right) \\
& +\mathbb{E}_{t}\left(\int_{t}^{t+h} \widetilde{\alpha}_{s}^{(i)} d s\right) .
\end{aligned}
$$

This expression for the discrete-time expected excess return maintains the same two-beta structure as the expression for the instantaneous risk premia in (6). ${ }^{16}$ It clearly highlights how the pricing of continuous and discontinuous market price risks may manifest differently in the cross-section of expected stock returns, and in turn how separately estimating $\beta_{t}^{(c, i)}$ and $\beta_{t}^{(d, i)}$ may allow for more accurate empirical predictions of the actual realized returns.

We turn next to a discussion of the new high-frequency based econometric procedures that we use for estimating the betas and investigate the separate pricing of the two different types of market price risks.

\section{Continuous and discontinuous beta estimation}

The decompositions of the prices for the market and each of the individual assets into separate diffusive and jump components that formally underly $\beta_{t}^{(c, i)}$ and $\beta_{t}^{(d, i)}$ in equations (1) and (2) above, are, of course, not directly observable. Instead, the different continuous-time price components, and in turn the betas, will have to be deduced from actually observed discrete-time prices and returns.

To this end, we will assume that high-frequency intraday prices are available at time grids of length $1 / n$ over the active intraday part of the trading day $[t, t+1)$. For notational simplicity, we will denote the corresponding logarithmic discrete-time return on the market over the $\tau^{\prime}$ th intraday time-interval by $r_{t: \tau}^{(0)} \equiv p_{t+\tau / n}^{(0)}-p_{t+(\tau-1) / n}^{(0)}$, with the $\tau^{\prime}$ th intraday return for asset $i$ defined accordingly as $r_{t: \tau}^{(i)} \equiv p_{t+\tau / n}^{(i)}-p_{t+(\tau-1) / n}^{(i)}$. The theory underlying our estimation is formally based on the notion of fill-in asymptotics and $n \rightarrow \infty$, or ever finer

\footnotetext{
${ }^{16}$ This contrast with the derivations in Longstaff (1989), who shows how temporally aggregating the simple continuous-time CAPM results in a multi-factor model, and the more recent paper by Corradi et al. (2013) which delivers conditional time-varying alphas and betas within a similar setting. Instead, our derivation is based on a general continuous-time jump-diffusion representation, and arrives at a consistent two-factor discrete-time pricing relation under the assumption that the separate jump and diffusive betas remain constant over the (short) return horizons.
} 
sampled high-frequency returns. ${ }^{17}$ To allow for reliable estimation, we will further assume that the betas stay constant over multi-day time-intervals of length $l>1$. $^{18}$

To begin, consider the estimation of the continuous betas. To convey the intuition, suppose that neither the market nor stock $i$ jumps, so that $\mu \equiv 0$ and $\mu^{(i)} \equiv 0$ almost surely. For simplicity, suppose also that the drift terms in (5) and (6) are both equal to zero, so that,

$$
r_{s: \tau}^{(i)}=\beta_{t}^{(i, c)} r_{s: \tau}^{(0)}+\widetilde{r}_{s: \tau}^{(i)}, \quad \text { where } \quad \widetilde{r}_{s: \tau}^{(i)} \equiv \int_{s+(\tau-1) / n}^{s+\tau / n} \widetilde{\sigma}_{u}^{(i)} d W_{u}^{(i)},
$$

for any $s \in[t-l, t]$. Thus, in this situation, the continuous beta may simply be estimated by an OLS regression of the discrete-time high-frequency returns for stock $i$ on the highfrequency returns for the market. Using a standard "polarization" of the covariance term, the resulting regression coefficient may be expressed as,

$$
\frac{\sum_{s=t-l}^{t-1} \sum_{\tau} r_{s: \tau}^{(i)} r_{s: \tau}^{(0)}}{\sum_{s=t-l}^{t-1} \sum_{\tau}\left(r_{s: \tau}^{(0)}\right)^{2}} \equiv \frac{\sum_{s=t-l}^{t-1} \sum_{\tau}\left[\left(r_{s: \tau}^{(i)}+r_{s: \tau}^{(0)}\right)^{2}-\left(r_{s: \tau}^{(i)}-r_{s: \tau}^{(0)}\right)^{2}\right]}{4 \sum_{s=t-l}^{t-1} \sum_{\tau}\left(r_{s: \tau}^{(0)}\right)^{2}}
$$

In general, of course, the market and stock $i$ may both jump over the $[t-l, t]$ time-interval, and the drift terms are not identically equal to zero. Meanwhile, it follows readily by standard arguments that for $n \rightarrow \infty$, the impact of the drift terms are asymptotically negligible. However, to allow for the possible occurrence of jumps, the simple estimator defined above needs to be appropriately modified by removing the discontinuous components. The "polarization" of the covariance provides a particularly convenient way of doing so by expressing the estimator in terms of sample portfolio variances. In particular, as shown by Todorov and Bollerslev (2010), the truncation-based estimator defined by, ${ }^{19}$

$$
\widehat{\beta}_{t}^{(c, i)}=\frac{\sum_{s=t-l}^{t-1} \sum_{\tau=1}^{n}\left[\left(r_{s: \tau}^{(i)}+r_{s: \tau}^{(0)}\right)^{2} \mathbf{1}_{\left\{\left|r_{s: \tau}^{(i)}+r_{s: \tau}^{(0)}\right| \leq k_{s, \tau}^{(i+0)}\right\}}-\left(r_{s: \tau}^{(i)}-r_{s: \tau}^{(0)}\right)^{2} \mathbf{1}_{\left\{\left|r_{s: \tau}^{(i)}-r_{s: \tau}^{(0)}\right| \leq k_{s, \tau}^{(i) 0)}\right\}}\right]}{4 \sum_{s=t-l}^{t-1} \sum_{\tau=1}^{n}\left(r_{s: \tau}^{(0)}\right)^{2} \mathbf{1}_{\left\{\left|r_{s: \tau}^{(0)}\right| \leq k_{s, \tau}^{(0)}\right\}}}
$$

consistently estimates the continuous beta for $n \rightarrow \infty$ under very general conditions.

\footnotetext{
${ }^{17}$ As discussed further below, a host of practical market microstructure complications invariably prevents us from sampling too finely. To assess the sensitive of our results to the specific choice of $n$, we experimented with the use of several different sampling schemes, including ones in which $n^{(i)}$ varies across stocks.

${ }^{18}$ Due to the relatively rare nature of jumps, in our main empirical results, we will base the estimation on a full year. However, as discussed further below, we also experimented with the use of shorter estimation periods, if anything, resulting in even stronger results and more pronounced patterns.

${ }^{19}$ In the empirical analysis below we follow Bollerslev et al. $(2013)$ in setting $k_{t, \tau}^{(\cdot)}=3 \times n^{-0.49}\left(R V_{t}^{(\cdot)} \wedge\right.$ $\left.B V_{t}^{(\cdot)} \times T O D_{\tau}^{(\cdot)}\right)^{1 / 2}$, where $R V_{t}^{(\cdot)}$ and $B V_{t}^{(\cdot)}$ denote the so-called realized variation and bipower variation on day $t$, respectively, and $T O D_{\tau}^{(\cdot)}$ refers to an estimate of the intraday Time-of-Day volatility pattern.
} 
Next, consider the estimation of the discontinuous beta. Assuming that $\beta_{t}^{(d, i)}$ is positive, it follows that for any $s \in[t-l, t]$ such that $\Delta p_{s}^{(0)} \neq 0$, the discontinuous beta is uniquely identified by,

$$
\beta_{t}^{(d, i)} \equiv \sqrt{\frac{\left(\Delta p_{s}^{(i)} \Delta p_{s}^{(0)}\right)^{2}}{\left(\Delta p_{s}^{(0)}\right)^{4}}} .
$$

Moreover, assuming that the beta is constant over the $[t-l, t]$ time-interval, this same ratio holds true for all of the market jumps that occurred between time $t-l$ and $t$. The actually observed high-frequency returns, of course, contain both diffusive and jump risk components. However, by raising the high-frequency returns to powers of order greater than two (four in the expression above), the diffusive martingale components become negligible, so that the systematic jumps dominate asymptotically for $n \rightarrow \infty .{ }^{20}$ This naturally suggests the following sample analogue to the expression for $\beta_{t}^{(d, i)}$ above as an estimator for the discontinuous beta, ${ }^{21}$

$$
\widehat{\beta}_{t}^{(d, i)}=\sqrt{\frac{\sum_{s=t-l}^{t-1} \sum_{\tau=1}^{n}\left(r_{s: \tau}^{(i)} r_{s: \tau}^{(0)}\right)^{2}}{\sum_{s=t-l}^{t-1} \sum_{\tau=1}^{n}\left(r_{s: \tau}^{(0)}\right)^{4}}} .
$$

As formally shown in Todorov and Bollerslev (2010), this estimator is indeed consistent for $\beta_{t}^{(d, i)}$ for $n \rightarrow \infty$.

The continuous-time processes in (1) and (2) underlying the definitions of the separate betas portray the prices as continuously evolving over time. In practice, of course, we only have access to high-frequency prices for the active part of the trading day when the stock exchanges are officially open. It is natural to think of the change in the price from the close on day $t$ to the opening on day $t+1$ as a discontinuity, or a "jump." 22 As such, the general continuous-time setup discussed in the previous section needs to be augmented with a separate jump term and jump beta measure $\beta_{t}^{(n, i)}$ accounting for the over-night comovements. The notion of an ever-increasing number of observations for identifying the intraday discontinuous price moves underlying the $\widehat{\beta}_{t}^{(d, i)}$ estimator in (9) does, of course,

\footnotetext{
${ }^{20}$ The basic idea of relying on higher order powers of returns to isolate the jump component of the price has previously been used in many other situations, both parametrically and nonparametrically; see, e.g., Barndorff-Nielsen and Shephard (2003) and Aït-Sahalia (2004).

${ }^{21}$ Since the sign of the jump betas gets lost by this transformation, our actual implementation also involves a sign correction, as detailed in Todorov and Bollerslev (2010). From a practical empirical perspective this is immaterial, as all of the estimated jump betas in our sample are positive.

${ }^{22}$ This characterization of the overnight returns as discontinuous movements occurring at deterministic times mirrors the high-frequency modeling approach recently advocated by Andersen et al. (2011).
} 
not apply with the over-night jump returns. However, $\beta_{t}^{(n, i)}$ may be similarly estimated by applying the same formula to all of the $l$ overnight jump return pairs.

In addition to the high-frequency based separate intraday and overnight betas, we also calculate standard regression-based CAPM betas for each of the individual stocks, say $\widehat{\beta}_{t}^{(s, i)}$. These are simply obtained by regressing the $l$ daily returns for stock $i$ on the corresponding daily returns for the market. In the following, we will refer to each of these four different beta estimates for stock $i$ without the explicit time subscript and hat as $\beta_{i}^{c}, \beta_{i}^{d}, \beta_{i}^{n}$, and $\beta_{i}^{s}$ for short. We turn next to a more detailed discussion of the data that we use in implementing these different estimators.

\section{Data and variables}

We begin this section with a discussion of the high-frequency data that we use in our analysis, followed by a discussion of the key properties of the resulting beta estimates. We also briefly discuss the other explanatory variables and controls that we use in our double portfolio sorts and cross-sectional pricing regressions.

\subsection{Data}

The individual stocks included in our analysis are comprised of the 985 constituents of the S\&P 500 index over the January 1993 to December 2010 sample period. All the highfrequency data for the individual stocks are obtained from the Trade and Quote (TAQ) database. The TAQ database provides all the necessary information to create our dataset containing second-by-second observations of trading volume, number of trades, and transaction prices between 9:30am and 4:00pm EST for the 4,535 trading days in the sample. ${ }^{23}$ We rely on high-frequency intraday S\&P 500 futures prices from Tick Data Inc. as our proxy for the aggregate market portfolio.

Our cleaning rule for the TAQ data follows Barndorff-Nielsen et al. (2009). It consists of two main steps: removing and assigning. The removing step filters out recording errors in prices and trade sizes. This step also deletes data points that TAQ flags as "problematic." The assigning step ensures that every second of the trading day has a single price. Additional details are provided in Appendix A.1.

The sample consists of 738 stocks per month on average. Altogether, these stocks account for approximately three-quarters of the total market capitalization of the entire stock universe in the Center for Research in Securities Prices (CRSP) database. Average daily trading

\footnotetext{
${ }^{23}$ The original dataset on average consists of more than 17 million observations per day for each trading day.
} 
volume for each stock increases from 302,026 in 1993 to 5,683,923 in 2010. Similarly, the daily number of trades for each stock rises from an average of 177 in 1993 to 20,197 in 2010. Conversely, the average trade size declines from 1,724 shares per trade in 1993 to just 202 in $2010 .^{24}$

We supplement the TAQ data with data from CRSP on total daily and monthly stock returns, number of shares outstanding, and daily and monthly trading volumes for each individual stock. To guard against survivorship biases associated with delistings, we take the delisting return from CRSP as the return on the last trading day following the delisting of a particular stock. We also use stock distribution information from CRSP to adjust overnight returns computed from the high-frequency prices. ${ }^{25}$ We rely on Kenneth R. French's website for daily and monthly returns on the Fama-French-Carhart four-factor portfolios. Lastly, we use the Compustat database for book values and other accounting information required for some of the control variables.

\subsection{Beta estimation results}

Our main empirical results are based on monthly continuous, discontinuous, and overnight beta estimates for each of the individual stocks in the sample. We rely on a one-year rolling overlapping monthly estimation scheme to balance the number of observations available for the estimation with the possible temporal variation in the systematic risks. ${ }^{26}$ We also experiment with the use of shorter 3 - and 6-month estimation windows. If anything, as further discussed in Section 8 below, these shorter estimation windows tend to result in even stronger return-beta patterns than the ones from the one-year moving windows detailed below.

We rely on a fixed intraday sampling frequency of 75 minutes in our estimation of the continuous and jump betas, with the returns spanning 9:45am to 4:00pm. ${ }^{27}$ A 75 -minute sampling frequency may seem quite coarse compared to the 5-minute sampling frequency

\footnotetext{
${ }^{24}$ Additional details concerning the high-frequency data for the individual stocks are available in the supplementary appendix Bollerslev et al. (2014).

${ }^{25}$ The TAQ database provides only the raw prices without considering price differences before and after distributions. We use the variable, "Cumulative Factor to Adjust Price" (CFACPR), from CRSP to adjust the high-frequency overnight returns after a distribution.

${ }^{26}$ The use of a relatively long estimation period may be especially important for the discontinuous betas, as there may be few or even no systematic jumps for a particular stock during a particular month; see also the discussion in Todorov and Bollerslev (2010). Annual horizon moving windows are also commonly used for the estimation of traditional CAPM betas based on coarser daily or monthly observations, as in, e.g., Ang et al. (2006a) and Fama and French (2006).

${ }^{27}$ Starting the trading day at 9:45am ensures that on most days most stocks will have traded at least once by that time. Patton and Verardo (2012) adopt a similar trading day convention in their high-frequency based realized beta estimation.
} 
commonly advocated in the literature on realized volatility estimation; see, e.g., Andersen et al. (2001) and the survey by Hansen and Lunde (2006). Yet estimation of multivariate realized variation measures, including betas, is invariably plagued by additional market microstructure complications relative to the estimation of univariate realized volatility measures. Coarser sampling frequencies are often used as a simple way to guard against any biases induced by these complications; see, e.g, the discussion in Sheppard (2006) and Bollerslev et al. (2008), along with the survey by Barndorff-Nielsen and Shephard (2007). However, we also experiment with a number of other intraday sampling frequencies, ranging from 5 minutes to 3 hours, as well as a "mixed frequency" explicitly related to the trading activity of each of the individual stocks. As further detailed in Section 8 below, our key empirical findings remain robust across all of these different sampling schemes.

In parallel to our high-frequency-based estimates for $\beta^{c}, \beta^{d}$, and $\beta^{n}$, our estimates for the monthly standard CAPM $\beta^{s}$ s are based on rolling overlapping regressions of the daily returns for each of the individual stocks over the past year on the daily returns for the S\&P 500 market portfolio. ${ }^{28}$

Turning to the actual estimation results, Panel A in Figure 1 depicts kernel density estimates of the unconditional distributions of the four different betas averaged across time and stocks. The discontinuous and overnight betas both tend to be somewhat higher on average and also more right-skewed than the continuous and standard betas. ${ }^{29}$ At the same time, the figure also suggests that the continuous betas are the least dispersed of the four betas across time and stocks. Part of the dispersion in the betas, may, of course, be attributed to estimation errors. Based on the expressions derived in Todorov and Bollerslev (2010), the asymptotic standard errors for $\beta^{c}$ and $\beta^{d}$ averaged across all of the stocks and months in the sample equal 0.06 and 0.12 , respectively, compared to 0.14 for the conventional OLS-based standard errors for the $\beta^{s}$ estimates. ${ }^{30}$

Panel B of Figure 1 shows the autocorrelograms for the four different betas averaged

\footnotetext{
${ }^{28}$ As an alternative to the standard CAPM betas, we also investigated high-frequency realized betas as in Andersen et al. (2005, 2006). The cross-sectional pricing results for these alternative "standard" beta estimates are very similar to the ones reported for the standard daily CAPM betas. Further details on these additional results are available upon request.

${ }^{29}$ The value-weighted averages of all the different betas should be equal to unity when averaged across the exact 500 stocks included in the S\&P 500 index at a particular point in time. In practice, we are measuring the betas over non-trivial annual time-intervals, and the S\&P 500 constituents and their weights also change over time, so the averages will not be exactly equal to one. For example, the value-weighted averages for $\beta^{s}$, $\beta^{c}, \beta^{d}$ and $\beta^{n}$ based on the exact 500 stocks included in the index at the very end of the sample, equal 1.04, $0.98,1.01$, and 1.06 , respectively.

${ }^{30}$ Intuitively, the continuous beta estimator may be interpreted as a regression based on truncated highfrequency intraday returns. As such, the standard errors should be reduced by a factor of approximately $1 / \sqrt{n}$, relative to the standard errors for the standard betas based on daily returns, where $n$ denotes the number of intradaily observations used in the estimation, here $1 / \sqrt{5} \approx 2.33$.
} 
across stocks. The apparent kink in all four correlograms at the 11th lag is directly attributable to the use of overlapping annual windows in the monthly beta estimation. Still, the figure clearly suggests a higher degree of persistence in $\beta^{c}$ and $\beta^{s}$ than in $\beta^{d}$ and $\beta^{n}$. This complements the existing high-frequency based empirical evidence documenting that continuous variation for most financial assets tends to be much more persistent and predictable than variation due to jumps; see, e.g., Barndorff-Nielsen and Shephard (2004b, 2006), and Andersen et al. (2007a).

In order to visualize the temporal and cross-sectional variation in the different betas, Figure 2 shows the time series of equally weighted portfolio betas, based on monthly quintile sorts for each of the four different betas and all of the individual stocks in the sample. The variation in the $\beta^{s}$ and $\beta^{c}$ sorted portfolios in Panels $\mathrm{A}$ and $\mathrm{B}$ are evidently fairly close. The plots for the $\beta^{d}$ and $\beta^{n}$ quintile portfolios in Panels $\mathrm{C}$ and $\mathrm{D}$, however, are distinctly different and more dispersed than the standard and continuous beta quintile portfolios.

To further illuminate these relations, Table 1 reports the results from Fama-MacBeth style regressions for explaining the cross-sectional variation in the standard betas as a function of the variation in the three other betas. Consistent with the results in Figures 1 and 2, the continuous beta $\beta^{c}$ exhibits the highest explanatory power for $\beta^{s}$, with an average adjusted $R^{2}$ of 0.76 . The two jump betas $\beta^{d}$ and $\beta^{n}$ each explain $62 \%$ and $46 \%$ of the variation in $\beta^{s}$, respectively. Altogether, $81 \%$ of the cross-sectional variation in $\beta^{s}$ may be accounted for by the high-frequency betas, with $\beta^{c}$ having by far the largest and most significant effect.

The differences in information content of the betas also manifest in different relations with the underlying continuous and discontinuous price variation. Relying on the truncation rules discussed in Section 3, the intraday discontinuous variation and the overnight variation account for approximately $9 \%$ and $30 \%$ of the total variation at the aggregate market level. Applying the same truncation rule to the individual stocks, the discontinuous and overnight variation account for an average of $10 \%$ and $32 \%$, respectively, at the individual firm level. Meanwhile, when sorting the stocks according to the four different betas, the sorts reveal a clear monotonic relation between $\beta^{d}$ and the jump contribution and between $\beta^{n}$ and the overnight contribution, but an inverse relation between $\beta^{c}$ and the proportion of the total variation accounted for by jumps.

\subsection{Other explanatory variables and controls}

A long list of prior empirical studies have sought to relate the cross-sectional variation in stock returns to other explanatory variables and firm characteristics. To guard against some of the most prominent previously documented effects and anomalies vis-a-vis the standard 
CAPM, in the double portfolio sorts and cross-sectional regressions reported below, we explicitly control for: firm size (ME), book-to-market ratio (BM), momentum (MOM), reversal (REV), idiosyncratic volatility (IVOL), coskewness (CSK), cokurtosis (CKT), realized skewness (RSK), realized kurtosis (RKT), maximum daily return (MAX), and illiquidity (ILLIQ). Our construction of these different control variables follows standard procedures in the literature, as discussed in more detail in Appendix A.2.

Table 2 displays time-series averages of monthly firm-level cross-sectional correlations between the four different betas and the various explanatory variables listed above. All of the four betas are negatively related to book-to-market and positively correlated with momentum. The betas are also generally positively correlated with idiosyncratic volatility, and the two jump betas more strongly so. On the other hand, while $\beta^{s}$ and $\beta^{c}$ are both negatively correlated with illiquidity, $\beta^{d}$ and $\beta^{n}$ both appear to be positively related to illiquidity.

To help further gauge these relations, Table 3 reports the results from a series of simple single-sorts. At the end of each month, we sort stocks by each of their betas. We then form five equal-sized portfolios and compute the time-series averages of the various firm characteristics for the stocks within each of these quintile portfolios. Consistent with the results discussed above, the portfolio sorts reveal a strong positive relation between all of the four different betas. Meanwhile, it also follows from Panels C and D that stocks with higher $\beta^{d}$ and $\beta^{n}$ tend to be smaller firms, stocks with lower book-to-market ratios, higher momentum, and higher idiosyncratic volatility. Higher discontinuous and overnight betas also tend to be associated with higher illiquidity, while the differences in illiquidity between High- and Low-quintile portfolios for the continuous and standard beta sorts in Panels A and $\mathrm{B}$ are both negative. ${ }^{31}$

\section{Contemporaneous portfolio sorts}

We begin our empirical investigations pertaining to the pricing of different market price risks with an examination of the contemporaneous relation between individual stock returns and the different betas. These investigations are directly motivated by the theory in Section 2 and equation (7) in particular, which implies a contemporaneous relation between the different betas and the expected returns.

At the beginning of each month, we estimate the four different betas based on the previous twelve months returns. We then sort the stocks into quintile portfolios based on their betas and record the returns over the same 12-month period. Rebalancing monthly, we record the

\footnotetext{
${ }^{31}$ Bali et al. (2014) and $\mathrm{Fu}(2009)$ also report a negative relation between standard betas and illiquidity.
} 
excess returns on each portfolio, starting with the first portfolio formation period spanning the first full year of the sample, ending with the last full year of the sample. This approach directly mirrors the single portfolio sorts commonly employed in the literature (see, e.g. Ang et al., 2006a, among numerous other studies).

Table 4 reports the results for equal-weighted portfolios. The average monthly returns for portfolios sorted by the standard beta are shown in Panel A. Consistent with the standard CAPM, the average excess returns increase across the $\beta^{s}$ segments. ${ }^{32}$ The spread between the High- and Low- $\beta^{s}$ quintile portfolios is only weakly statistically significant, however. The results for the continuous beta portfolio sorts reported in Panel B are comparable, with the return spread and $t$-statistic for the High-Low $\beta^{c}$-sorted portfolios equal to $1.61 \%$ and 1.81 , respectively. By comparison, the results for the two rough beta sorts reported in Panels $\mathrm{C}$ and $\mathrm{D}$, respectively, both show a stronger and more reliable relation between the betas and the contemporaneous portfolio returns. For the $\beta^{d}$-sorts the return spread for the High-Low portfolio equals $1.71 \%$ with a $t$-statistic of 2.63 , while for the $\beta^{n}$-sorts the spread and the corresponding $t$-statistic equal $1.64 \%$ and 2.59 , respectively.

To more directly explore the idea that most of the premium for market price risks stem from the compensation for jump risk, Panels E and F report the results based on portfolios sorted by the relative betas $\beta^{d}-\beta^{s}$ and $\beta^{n}-\beta^{s}$, respectively. As evident from the almost flat $\beta^{s}$ loadings coupled with the increasing $\beta^{d}$ or $\beta^{n}$ loadings over the different quintiles, the relative betas effectively eliminate the part of the cross-sectional variation in each of the two jump betas that may be explained by the variation in the standard beta. ${ }^{33}$ Even though the spreads in the returns are smaller when sorting on these relative jump betas compared to the sorts based on the individual betas, the $t$-statistics equal to 3.34 and 3.18 are both higher than the $t$-statistics associated with any of individual beta sorts. As such, this clearly highlights the important differences in the risks measured by the jump betas and the standard betas and specifically the pricing thereof.

Table 5 further corroborates the findings discussed above, by reporting the results for value-weighted quintile portfolios. Although the return differences appear slightly muted relative to the results for the equally-weighted portfolios reported in Table 4, the same general relations between the betas and the returns continue to hold true. ${ }^{34}$ In particular,

\footnotetext{
${ }^{32}$ Note that even though the relationship is monotonic, most of the spread in the returns between the High and Low portfolios comes from the spread between the 4th and highest quintile. This is true for many of the other portfolio sorts discussed below as well.

${ }^{33}$ Similar relative beta measures have also been used by Ang et al. (2006a) in their study of downside beta risk, and by Bali et al. (2014) in their study of dynamic conditional betas.

${ }^{34}$ The finding that the jump beta risk manifest slightly weaker in value-weighted as opposed to equallyweighted portfolios is consistent with many other previously analyzed non-linearities in the cross-section of stock returns, as, e.g., the downside risk measure in Ang et al. (2006a) and the coskewness effect in Harvey
} 
the monthly excess return spreads between the High and Low quintiles for the $\beta^{d}$ and $\beta^{n}$ sorts equal to $1.56 \%$ and $1.60 \%$, respectively, are both statistically significant at the usual $5 \%$ significance level. On the other hand, the portfolios sorted by $\beta^{s}$ and $\beta^{c}$ both have lower return spreads, and the $t$-statistics for the differences are also insignificant. The relative beta sorts, designed to eliminate the variation in the standard beta from each of the two jump betas, further underscore that the discontinuous and overnight betas are more closely related to cross-sectional differences in the returns than the standard beta.

\section{Predictive portfolio sorts}

The portfolio sorts discussed in the previous section pertain to returns and betas estimated over the same holding period. While this represents the essence of the risk-return relationship implied by the theoretical framework in Section 2, these results are not of much practical value if the betas can not be used to predict future returns. In this section, we therefore extend the previous contemporaneous portfolio sorts to a predictive setting. We begin by discussing the results from simple single-sorted portfolios as in the previous section, followed by the results for various double-sorted portfolios that explicitly control for other explanatory variables.

\subsection{Single-sorted portfolio returns}

Table 6 summarizes the results from our predictive single-sorts. In parallel to the sorts discussed in the previous section, at the end of each month, we estimate the different betas based on the past twelve months returns. We then sort the stocks according to each of the different betas and record the returns for the following month. In addition to the resulting pre-formation beta estimates and the predictive ex-post excess returns for each of the equallyweighted quintile portfolios, we also report the risk-adjusted excess returns, as measured by the intercept from a time-series regression of the monthly portfolio returns on the four FamaFrench-Carhart factors, together with the ex-post betas for the different portfolios based on the beta estimates for the 12 months proceeding the pre-formation period.

Comparing the ex-post betas with the pre-formation beta estimates, the High-Low quintile spreads are naturally dampened somewhat relative to the ex-ante measures. However, consistent with the slowly decaying autocorrelations for the betas shown in Figure 2, the High-Low spreads remain quite sizeable for all of the four individual beta sorts in Panels A-D. The spreads in the ex-post relative betas for the $\beta^{d}-\beta^{s}$ and $\beta^{n}-\beta^{s}$ sorts reported and Siddique (2000), which tend to manifest more strongly in smaller stocks. 
in Panels E and F, respectively, are, not surprisingly, reduced more than the spreads in the ex-post betas for the individual beta sorts, but the spreads remain non-trivial.

This persistence in the betas translate into similar predictive return-beta relations to the ones documented for the contemporaneous return in the previous section. Specifically, we continue to see a monotone relationship between the future portfolio returns and the past betas. Directly in line with the previous contemporaneous portfolio sorts, the relations are stronger and more statistically significant for the rough betas than for the standard and continuous betas. In particular, focusing on the risk-adjusted FFC4 alphas, the $t$ statistics for the High-Low quintile portfolios based on the $\beta^{s}$ and $\beta^{c}$ sorts equal 1.76 and 1.44, respectively, compared to 2.04 and 2.74 for the $\beta^{d}$ and $\beta^{n}$ predictive sorts, while the $t$-statistics for the relative $\beta^{d}-\beta^{s}$ and $\beta^{n}-\beta^{s}$ sorts equal 2.29 and 3.05, respectively. ${ }^{35}$ Thus, not only do the results suggest that the discontinuous and overnight betas are better able to predict the cross-sectional variation in the future returns than the continuous and standard betas, these relations between the rough betas and the future returns cannot be explained by the size, book-to-market ratio, and momentum effects captured by the Fama-French-Carhart factors.

\subsection{Double-sorted portfolio returns}

Our single portfolio sorts reveal that stocks with high discontinuous and overnight betas tend to have high returns. The converse is true as well, and the differences are greater than the differences for stocks sorted by their continuous and standard betas. Yet, as discussed in Section 4.3, the cross-sectional variation in the discontinuous and overnight betas is also related to other firm characteristics and explanatory variables that are typically shown to help predict the cross-sectional variation in stock returns. Examination of double-sorted portfolios provides a simple approach to help disentangle these effects.

For each explanatory variable and each month in the sample, we sort all stocks into five quintiles according to a particular control variable. Within each quintile, we then sort stocks into five additional quintiles according to one of the four different beta measures. Finally, we average the returns on the five beta portfolios across the five different control variable portfolios to produce beta portfolios with large cross-portfolio variations in their betas, but little variation in the control variable.

\footnotetext{
${ }^{35}$ Our use of the lagged betas for the predictive portfolio sorts in Table 6 implicitly assumes that the true betas follow a random walk. It is possible that stronger predictive results could be obtained by the use of more sophisticated, possibly firm specific, time series models for forecasting the betas. We will not pursue that any further here. We did, however, experiment with use of recursively estimated simple AR(1) forecasting models, resulting in $t$-statistics for the FFC4 alphas for the two relative betas of 1.97 and 3.09 , respectively. Further details of these additional results are available upon request.
} 
Panels A-D in Table 7 display these double-sorting results for each of the different betas. In Panels $\mathrm{C}$ and $\mathrm{D}$ for the discontinuous and overnight betas, higher $\beta^{d}$ and $\beta^{n}$ are always associated with higher portfolio returns. For $\beta^{d}$ in particular, the spread in the returns between the High- and Low-quintile portfolios ranges from $0.48 \%$ (MOM) to $1.05 \%$ (RKT), while the spreads in the FFC4 alphas range from $0.26 \%$ (MOM) to $0.73 \%$ (REV and RKT). Similarly, for the $\beta^{n}$ portfolio sorts the spreads range from $0.65 \%$ (MOM) to $1.21 \%$ (RKT), while the spreads in the FFC4 alphas range from $0.43 \%$ (MOM) to $0.91 \%$ (RKT). Most of these alphas are not only statistically significant at the usual $5 \%$ level, but they also translate into economically meaningful differences, ranging from $0.26 \% \times 12=3.1 \%$ to $1.21 \% \times 12=14.5 \%$ per year. Comparison of the results across different betas in the four different panels for a given control variable also reveals that the High-Low portfolio return differences are generally the greatest for the $\beta^{d}$ and $\beta^{n}$ based double-sorts, further corroborating the idea that systematic jump risk is priced higher than continuous market price risk.

\section{$7 \quad$ Firm-level cross-sectional pricing regressions}

The portfolio sorts discussed so far impose no model assumptions. However, they ignore potentially important cross-sectional firm-level information by aggregating the stocks into quintile portfolios. Also, even though the double-sorted portfolios do control for other explanatory variables, they only control for one variable at a time. Hence, we turn next to a standard Fama and MacBeth (1973) type cross-sectional approach based on firm-level data for estimating the risk premiums associated with the different betas, while simultaneously controlling for multiple explanatory variables.

For ease of notation, let the unit time interval be a month. The cross-sectional pricing regression for each of the months $t=1,2, \ldots, T$, and all of the stocks $i=1,2, \ldots, N_{t}$ available for a particular month $t$ in the sample, may then be expressed as,

$$
r_{t, t+1}^{(i)}=\gamma_{0, t}+\gamma_{\beta, t}^{c} \beta_{t}^{(c, i)}+\gamma_{\beta, t}^{d} \beta_{t}^{(d, i)}+\gamma_{\beta, t}^{n} \beta_{t}^{(n, i)}+\sum_{j=1}^{p} \gamma_{j, t} Z_{j, t}^{(i)}+\epsilon_{t, t+1}^{(i)},
$$

where $r_{t, t+1}^{(i)}$ denotes the excess return for stock $i$ from month $t$ to month $t+1$, and the explanatory variables $Z_{j, t}^{(i)}$ and the betas $\beta_{t}^{(c, i)}, \beta_{t}^{(d, i)}$, and $\beta_{t}^{(n, i)}$ are measured at the end of month $t .{ }^{36}$ For comparison, we also estimate similar regressions by replacing the three betas

\footnotetext{
${ }^{36}$ Following common practice in the literature (e.g., Ang et al., 2006a, among many others), in an effort to reduce the effect of extreme observations or outliers, we Winsorize the independent variables at their $0.5 \%$ and $99.5 \%$ levels. The results from the non-Winsorized regressions, available upon request, are very similar to the results reported here.
} 
by the standard CAPM beta $\beta_{t}^{(s, i)}$. Based on these cross-sectional regression results, we then estimate the risk premiums associated with the different betas and explanatory variables as the time-series means of the $T=204$ individual monthly gamma estimates. Specifically, for $k=s, c, d, n$ and $j=1, \ldots, p$,

$$
\hat{\gamma}_{\beta}^{k}=\frac{1}{T} \sum_{t=1}^{T} \hat{\gamma}_{\beta, t}^{k}, \quad \text { and } \quad \hat{\gamma}_{j}=\frac{1}{T} \sum_{t=1}^{T} \hat{\gamma}_{j, t} .
$$

The average risk premium estimates, with robust $t$-statistics in parentheses, are reported in Table 8 for a range of different combinations of explanatory variables. Panel A gives the results from simple univariate regressions involving a single beta measure or a single explanatory variable. Consistent with the standard CAPM, all the beta risk premiums are estimated to be positive. The premium associated with $\beta^{c}$ is the highest of the four, and that of $\beta^{n}$ is the lowest, although the $t$-statistic associated with $\beta^{n}$ is actually the highest and the $t$-statistic with $\beta^{c}$ the lowest. Many of the previously documented CAPM-related anomalies appear fairly weak in the present sample of relatively large liquid stocks. Still, the significant positive premiums for ILLIQ and RKT do corroborate the empirical findings in Amihud (2002) and Amaya et al. (2013), respectively. The negative estimates for ME, CSK, and RSK are also in line with the empirical evidence reported in Fama and French (1992), Harvey and Siddique (2000), and Amaya et al. (2013), among many others. Meanwhile, the positive albeit statistically weak premium for IVOL is counter to the idiosyncratic volatility puzzle first highlighted by Ang et al. (2006b). However, as previously documented in the literature, the idiosyncratic volatility puzzle is primarily driven by small firms (Fu, 2009), firms that are dominated by retail investors (Han and Kumar, 2013), and lottery-like firms (Bali et al., 2011). ${ }^{37}$ By contrast, our sample of S\&P 500 constituents consists entirely of relatively large firms.

Turning to the multiple regression results in Panel B, Regression I shows that the standard beta $\beta^{s}$ becomes insignificant when controlling for all the other explanatory variables. Similarly, the risk premium for $\beta^{c}$ in Regression II is also insignificant, suggesting that the explanatory power of the continuous beta is effectively subsumed by the other explanatory variables. The $t$-statistic for the discontinuous beta $\beta^{d}$ in Regression III, however, is largely unchanged from the results in the simple regression in Panel A. For the overnight beta $\beta^{n}$ the $t$-statistic for Regression IV is even higher than in the simple regression in Panel A.

Regressions V - XIII show the results from simultaneously including the continuous, discontinuous, and overnight betas, controlling for ME, BM, MOM and each of the other

\footnotetext{
${ }^{37}$ For a recent discussion of the idiosyncratic volatility puzzle see also Stambaugh et al. (2014), who find that the IVOL effect is most pronounced and negative for portfolios comprised of overvalued stocks, but actually positive for portfolios of undervalued stocks.
} 
explanatory variables in turn. The high correlations across the different beta estimates, previously discussed in Table 2, invariably render lower slope coefficients and $t$-statistics than in Regressions II - IV. Nonetheless, the estimated risk premiums associated with $\beta^{d}$ and $\beta^{n}$ remain close to significant across all the specifications when judged by their one-sided $t$-statistics at the usual $5 \%$ level. The $t$-statistics for $\beta^{c}$, on the other hand, are practically zero for all specifications, suggesting that the premium for systematic continuous market risk is fully absorbed by the premiums for the two rough betas and the other explanatory variables.

The estimated premiums for $\beta^{d}$ and $\beta^{n}$ risks are also remarkably robust across the different specifications, with typical values of around $0.3 \%$ for each of the rough betas. Indeed, the $t$-statistic for testing that the two premiums are the same after controlling for all the other explanatory variables equals just 0.26 . Hence, in Regression XIV, we report the results including the three betas and all control variables, explicitly restricting the premiums for $\beta^{d}$ and $\beta^{n}$ risks to be the same. The estimated common rough beta risk premium equals $0.31 \%$ with a $t$-statistic of 2.33 . Given that the cross-sectional standard deviations of $\beta^{d}$ and $\beta^{n}$ are equal to 1.14 and 1.20, respectively, a two-standard deviation change in each of the two rough betas also translates into large and economically meaningful expected return differences of about $2 \times 1.14 \times 0.33 \% \times 12=9.03 \%$ and $2 \times 1.20 \times 0.33 \% \times 12=9.50 \%$ per year, respectively.

The last Regression XV further constrains all three $\beta^{c}, \beta^{d}$, and $\beta^{n}$ risks to have the same premium. This results in a marginally significant $t$-statistic of 1.96 for the beta risk premium. However, a standard $F$-test easily rejects the null hypothesis that the three risk premiums are the same. By contrast, the assumption that the risk premiums for $\beta^{d}$ and $\beta^{n}$ are the same and different from the premium for $\beta^{c}$, as in Regression XIV, cannot be rejected.

\section{Robustness checks}

To further help corroborate the robustness of our findings, we carry out a series of additional tests and empirical investigations. To begin with, we investigate the sensitivity of our primary empirical findings to the choice of intraday sampling frequency, possible biases in the estimation of the betas induced by non-synchronous trading effects, and errors-in-variables problems in the cross-sectional regressions stemming from the estimation errors in the betas. Next, we analyze how the cross-sectional regression results and the estimated risk premiums for different betas are affected by the length of the sample period used in the estimation of the betas and the holding period of the future returns. We then show how the rough betas 
also help reduce the conditional alphas and the spread in the conditional alphas sorted by various characteristics compared to the alphas for a standard conditional CAPM. Finally, we compare our main results to those obtained by excluding specific macroeconomic news announcement days in the estimation of the betas.

\subsection{Sampling frequency and beta estimation}

The continuous-time framework of the empirical investigations and the consistency of the $\beta^{c}$ and $\beta^{d}$ estimates hinge on increasingly finer sampled intraday returns. In practice, nonsynchronous trading and other market microstructure effects invariably limit the frequency of the data available for estimation. To assess the sensitivity of the beta estimates to the choice of sampling frequency, we compute betas for five different fixed sampling frequencies: 5-, 25-, 75-, 125- and 180-minute. These five sampling schemes, ranging from a total of 75 observations per day (5-minute) to only two observations per day (180-minute), span most of the frequencies used in the literature for computing multivariate realized variation measures. The extent of market microstructure frictions obviously varies across different stocks. Less frequently traded stocks are likely more prone to estimation biases in their betas from too frequent sampling than more liquid stocks. Thus, we also adopt a mixed-frequency strategy in which we apply different sampling frequencies to different stocks. Specifically, at the end of each month $t$, we sort all stocks into quintiles according to their ILLIQ measure. We then use the $i$ th highest of the five fixed sampling frequencies for stocks in the $i$ th illiquidity quintile; i.e., 5-minute frequency for stocks in the lowest ILLIQ quintile (the most liquid) and 180-minute frequency for stocks in the highest ILLIQ quintile (the least liquid).

Figure 3 plots the sample means averaged across time and stocks for the resulting $\beta^{c}$ and $\beta^{d}$ estimates as a function of the five different fixed sampling frequencies. The sample means of the mixed-frequency beta estimates are shown as a flat dashed line in both panels. The average $\beta^{c}$ estimates, reported in Panel A, increase quite substantially from the 5- to the 25minute sampling frequency, but appear to flatten at around 0.93 at the 75 -minute sampling frequency used in our empirical results reported so far. The average $\beta^{d}$ estimates reported in Panel B, however, are remarkably stable across different sampling frequencies and close to the average mixed-frequency value of 1.35. The specific choice of sampling frequency within the range of values considered here appears largely irrelevant to the two discontinuous beta estimates.

To further investigate the role of sampling frequency in our key empirical findings, Table 9 reports results of cross-sectional pricing regressions based on the different beta estimates. Panel A gives the results obtained by varying the sampling frequency used in the estimation 
of $\beta^{c}$, keeping the sampling frequency for the $\beta^{d}$ estimation fixed at 75 minutes. Panel B reports the results for the different $\beta^{d}$ estimates, using the same 75 -minute $\beta^{c}$ estimates. To conserve space, we report only results corresponding to the full Regression XIV reported in Panel B of Table 8 that restricts the premiums for the two rough betas to be the same.

None of the $t$-statistics for the continuous systematic risk premiums in Panel A are close to significant. All of the $t$-statistics for the rough beta risk premiums, on the other hand, are higher than two. The estimated risk premiums are also very similar across the different regressions and close to the value of $0.31 \%$ for the benchmark Regression XIV in Table 8. The regressions in Panel $\mathrm{B}$ for the different $\beta^{d}$ estimates tell a very similar story. The risk premiums for the rough betas are always significant, while those for the continuous betas are not. Overall, our key cross-sectional pricing results appear robust to choice of intraday sampling frequency used in the estimation of the $\beta^{c}$ and $\beta^{d}$ risk measures.

\subsection{Non-synchronous trading and beta estimation}

The results in the previous section indicate that the estimated jump betas are very stable across different sampling frequencies, while the continuous betas appear to be downward biased for the highest sampling frequencies. This downward bias may in part be attributed to non-synchronous trading effects. In order to more directly investigate this, following the original ideas of Scholes and Williams (1977) and Dimson (1979), we calculate high-frequency based lead and lag continuous betas as,

$\widehat{\beta}_{t,-}^{(c, i)}=\frac{n}{n-1} \frac{\sum_{s=t-l}^{t-1} \sum_{\tau=2}^{n}\left[\left(r_{s: \tau}^{(i)}+r_{s: \tau-1}^{(0)}\right)^{2} \mathbf{1}_{\left\{\left|r_{s: \tau}^{(i)}+r_{s: \tau-1}^{(0)}\right| \leq k_{s, \tau}^{(i+0)}\right\}}-\left(r_{s: \tau}^{(i)}-r_{s: \tau-1}^{(0)}\right)^{2} \mathbf{1}_{\left\{\left|r_{s: \tau}^{(i)}-r_{s: \tau-1}^{(0)}\right| \leq k_{s, \tau}^{(i-0)}\right\}}\right]}{4 \sum_{s=t-l}^{t-1} \sum_{\tau=1}^{n}\left(r_{s: \tau}^{(0)}\right)^{2} \mathbf{1}_{\left\{\left|r_{s: \tau}^{(0)}\right| \leq k_{s, \tau}^{(0)}\right\}}}$,

and

$\widehat{\beta}_{t,+}^{(c, i)}=\frac{n}{n-1} \frac{\sum_{s=t-l}^{t-1} \sum_{\tau=2}^{n}\left[\left(r_{s: \tau-1}^{(i)}+r_{s: \tau}^{(0)}\right)^{2} \mathbf{1}_{\left\{\left|r_{s: \tau-1}^{(i)}+r_{s: \tau}^{(0)}\right| \leq k_{s, \tau}^{(i+0)}\right\}}-\left(r_{s: \tau-1}^{(i)}-r_{s: \tau}^{(0)}\right)^{2} \mathbf{1}_{\left\{\left|r_{s: \tau-1}^{(i)}-r_{s: \tau}^{(0)}\right| \leq k_{s, \tau}^{(i-0)}\right\}}\right]}{4 \sum_{s=t-l}^{t-1} \sum_{\tau=1}^{n}\left(r_{s: \tau}^{(0)}\right)^{2} \mathbf{1}_{\left\{\left|r_{s: \tau}^{(0)}\right| \leq k_{s, \tau}^{(0)}\right\}}}$,

where $n$ denotes the number of high-frequency observations within a day used in the estimation; i.e., $n=5$ for the 75-minute sampling underlying our main empirical results. The theory behind the high-frequency betas implies that the lead and lag betas should be asymptotically negligible and thus have no significant impact on the cross-sectional pricing. ${ }^{38}$

\footnotetext{
${ }^{38}$ It is not possible to similarly adjust the jump betas by including leads and lags in their calculation. The lead-lag adjustment for the continuous betas rely on the notion that the "true" high-frequency returns are approximately serially uncorrelated. However, the construction of the jump betas is based on higher order powers of the high-frequency returns, and the squared returns, in particular, are clearly not serially
} 
To test for this, we repeat the one-month ahead predictive single sorts in Table 6 , by instead sorting the stocks according to their $\widehat{\beta}_{t,-}^{(c, i)}$ and $\widehat{\beta}_{t,+}^{(c, i)}$ estimates. The monthly FFC4 alphas for the return differences between the resulting High and Low quintile portfolios equal $-0.07 \%$ with a $t$-statistic of -0.30 for the lagged continuous beta sorts, and $0.05 \%$ with a $t$-statistic of 0.30 for the lead continuous beta sorts, thus corroborating the idea that neither the lead nor the lagged continuous betas are priced in the cross-section.

To more directly assess whether microstructure biases, and non-synchronous trading effects in particular, systematically affect the previously estimated continuous betas and the pricing thereof, we also calculate an adjusted continuous beta by adding all three continuous beta estimates, $\widehat{\beta}_{t, a d j}^{(c, i)} \equiv \widehat{\beta}_{t}^{(c, i)}+\widehat{\beta}_{t,-}^{(c, i)}+\widehat{\beta}_{t,+}^{(c, i)}$. Sorting by these adjusted continuous betas produces an FFC4 alpha for the spread in the returns between the High and Low quintile portfolios of $0.55 \%$, with a $t$-statistic of 1.66 , very close to the values reported in Table $6 .{ }^{39}$

Taken as a whole, the results discussed in the previous section together with the results for the lead-lag beta adjustments discussed above, indicate that non-synchronous trading effects and biases in the high-frequency betas are not of great concern.

\subsection{Errors-in-variables in the cross-sectional pricing regressions}

Another potential concern when testing linear factor pricing models relates to the errorsin-variables problem arising from the first-stage estimation of the betas. In particular, as formally shown by Shanken (1992), the first-stage estimation error generally results in an increase in the asymptotic variance of the risk premia estimates from the second-stage crosssectional regressions. In our setting, however, the betas are estimated from high-frequency data, resulting in lower measurement errors, and in turn less of an errors-in-variables problem, than in traditional Fama-MacBeth type regressions that rely on betas estimated with lower frequency data. At the same time, this also means that the standard adjustment procedures, as in, e.g., Shanken (1992), are not applicable in the present context. ${ }^{40}$

Instead, we conduct a small-scale Monte Carlo experiment by appropriately perturbing the high-frequency beta estimates. For $\widehat{\beta}_{t}^{(c, i)}$ and $\widehat{\beta}_{t}^{(d, i)}$, we rely on the results in Todorov and

uncorrelated. Meanwhile, the "signature plots" for the jump betas in Panel B of Figure 3 discussed in the previous section, show that the estimates of the jump betas are very robust to the choice of sampling frequency, and as such much less prone to any systematic biases arising from non-synchronous trading effects.

${ }^{39}$ Adjusting the standard beta by similarly adding the lead and lag betas, results in an FFC4 alpha for the High minus Low portfolio of $0.56 \%$ with a $t$-statistic of 1.76 , both of which are almost the same as the values for the unadjusted standard beta sort reported in Table 6 .

${ }^{40}$ Formally accounting for the estimation errors in the high-frequency betas would require a new asymptotic framework in which both the time span of the data used for the cross-sectional regression-based estimates of the risk premia and the sampling frequency used for the estimation of the betas go to infinity. We leave this for future work. 
Bollerslev (2010) to generate replicates $\left\{\widehat{\beta}_{t}^{(c, i, r e p)}\right\}$ and $\left\{\widehat{\beta}_{t}^{(d, i, r e p)}\right\}$ from two independent normal distributions with means equal to the estimated $\widehat{\beta}_{t}^{(c, i)}$ and $\widehat{\beta}_{t}^{(d, i)}$, respectively, and standard deviations equal to the corresponding theoretical asymptotic standard errors. For $\widehat{\beta}_{t}^{(n, i)}$, we rely on a bootstrap procedure to generate random samples of $\widehat{\beta}_{t}^{(n, i, r e p)}$ from the actual sampling distribution. Given a random sample of the three betas $\left(\widehat{\beta}_{t}^{(c, i, r e p)}, \widehat{\beta}_{t}^{(d, i, r e p)}, \widehat{\beta}_{t}^{(n, i, r e p)}\right)$, we then estimate the key Fama-MacBeth Regression XIV in Table 8 based on the perturbed beta estimates keeping all of the other controls the same. We repeat the simulations a total of one-hundred times.

The resulting simulation-based estimates for the risk premia are in the range of -0.12 to 0.27 for $\beta^{c}$ with $t$-statistic between -0.24 and 0.87 , and in the range of 0.20 to 0.38 with $t$ statistic between 1.62 and 3.16 for $\beta^{d}$ and $\beta^{n}$. The magnitudes of these simulated risk premia and their $t$-statistics are all fairly close to the values for the actual regression reported in Table 8, thus confirming that the errors-in-variables problem is not of major concern in the present context, and that it does not materially affect the statistical nor economic significance of the rough betas.

\subsection{Beta estimation and return holding periods}

All the cross-sectional pricing regressions in Tables 8 and 9 are based on betas estimated from returns over the past year and a future one-month return holding period. These are typical estimation and holding periods used to test for the significant pricing ability of explanatory variables and risk factors. To assess the robustness of our results to different lagged beta estimation periods (L) and longer future return horizons $(\mathrm{H})$, Table 10 reports results based on shorter 3- and 6-month beta estimates and longer 3-, 6- and 12-month prediction horizons. ${ }^{41}$

Regressions I-V pertain to the standard beta. Although the regression coefficients associated with the standard beta seem to increase with the forecast horizon, their $t$-statistics are at most weakly significant. Regressions VI-X pertain to the continuous and rough betas. The regressions show that the $t$-statistics associated with the two rough betas are always significant, while the continuous systematic risk is not priced in the cross-section. In fact, if anything, the results for the shorter estimation periods and longer return horizons are even more significant than the results for the baseline Regression XIV in Panel B of Table 8 and the typical choice of $\mathrm{L}=12$ and $\mathrm{H}=1$.

The significance of the results for the longer 3-, 6-, and 12-month return horizons also

\footnotetext{
${ }^{41} \mathrm{All}$ of the cross-sectional regressions are estimated monthly. The robust $t$-statistics for the longer $\mathrm{H}=$ 3-, 6- and 12-month return horizons explicitly adjust for the resulting overlap in the return observations.
} 
highlights non-trivial persistence in the cross-sectional predictability. Converting the resulting estimates for the different return horizons to an annual level implies rough beta risk premiums of $1.65 \% \times 4=6.60 \%, 2.73 \% \times 2=5.46 \%$, and $4.07 \times 1=4.07 \%$ per year, respectively, compared to $0.31 \times 12=3.72 \%$ per year for the one-month future return horizon implied by Regression XIV in Table 8.

It is also worth noting that while ME, REV, and RKT are each significant in one or more of the regressions reported in Table 10, they are not systematically so. Short-term reversal and realized kurtosis, in particular, both lose their significance for the longer 6- and 12month holding periods. The only variable that remains highly significant across all different estimation periods and return predictability horizons is the rough beta risk premium.

\subsection{Conditional alphas}

As an additional robustness check, we investigate to what extent our separation of market diffusive and jump price risks can reduce, if at all, the conditional alphas generated by the standard conditional CAPM that treats all market price moves the same. To this end, we define the conditional alpha for stock $i$ from time $t$ to $t+1$ in our generalized setting as,

$$
\alpha_{t, t+1}^{(i)} \equiv r_{t, t+1}^{(i)}-\beta_{t}^{(c, i)} \gamma_{\beta, t}^{c}-\beta_{t}^{(d, i)} \gamma_{\beta, t}^{d}-\beta_{t}^{(n, i)} \gamma_{\beta, t}^{n}
$$

If market diffusive and jump price risks were the only systematic risks that were priced, $\alpha_{t, t+1}^{(i)}$ should be a martingale difference sequence; i.e., $\mathbb{E}_{t}\left(\alpha_{t, t+1}^{(i)}\right)=0$. However, as formally discussed in Section 2, other risk factors might be priced as well. As such, we are not interested in testing the martingale hypothesis per se, but rather if the magnitude of the generalized alphas are reduced relative to the standard conditional CAPM alphas that do not differentiate between market diffusive and jump price moves.

The betas and the risk premia, and in turn the conditional alphas defined in equation (12) are not directly observable. The betas, of course, may be accurately estimated from highfrequency intraday data. The estimation of the conditional risk premia, however, are more complicated. As discussed in Lewellen and Nagel (2006), conditionally unbiased estimates of the risk premia generally suffice for the estimation of the mean of the conditional alphas, as the errors in the risk premia estimates are "averaged out" in the time-series average of the alpha estimates. This is easily accomplished for the standard conditional CAPM by taking the difference between the excess return of the asset and the product of its conditional market beta and the excess market return. This approach, however, does not extend to our generalized setup, as the market diffusive and jump risks are latent components of the total market return, and as such their risk premia are not associated with the returns on any directly observable factors. 
Instead, mirroring the approach in Section 7, we estimate $\gamma_{\beta, t}^{c}, \gamma_{\beta, t}^{d}$ and $\gamma_{\beta, t}^{n}$ from crosssectional regressions of the excess returns on the high-frequency beta estimates. Further motivated by the empirical findings in Section 7 and the benchmark Regression XIV in Table 8 , we restrict the premia for $\beta_{t}^{(d, i)}$ and $\beta_{t}^{(n, i)}$ to be the same, resulting in the following generalized conditional alpha estimates,

$$
\widehat{\alpha}_{t, t+1}^{(i)}=r_{t, t+1}^{(i)}-\widehat{\beta}_{t}^{(c, i)} \widehat{\gamma}_{\beta, t}^{c}-\left(\widehat{\beta}_{t}^{(d, i)}+\widehat{\beta}_{t}^{(n, i)}\right) \widehat{\gamma}_{\beta, t}^{d}
$$

Assuming that the size of the cross-section is large relative to the length of the time series, so that the estimation error in recovering the risk premia has no first-order asymptotic effect, standard errors for the time-series averages of the alphas $\widehat{\alpha}^{(i)}=\frac{1}{T} \sum_{t=1}^{T} \widehat{\alpha}_{t, t+1}^{(i)}$ are easily constructed from the time series variance of the conditional alphas. Our estimation of the conditional alphas for the standard conditional CAPM is carried out analogously.

Figure 4 displays the cross-sectional distributions of $\widehat{\alpha}^{(i)}$ corresponding to the standard conditional CAPM and the generalized conditional alphas in (13). As is evident from the figure, the distribution of the generalized conditional alphas is more centered around zero than the standard conditional CAPM alphas. Specifically, while the cross-sectional average of the average conditional alphas equals $0.44 \%$ per month for the standard conditional CAPM, the average drops to $-0.03 \%$ per month when allowing for separate pricing of market diffusive and jump price risks. Even though there is a similar drop in the values of the $t$-tests for testing the hypothesis that the average conditional alphas are equal to zero, few of the $t$-statistics for the individual stocks are actually significant at the usual $5 \%$ level.

In order to more clearly highlight the improvements afforded by treating diffusive and jump risks differently, we therefore also consider two aggregate portfolios where the idiosyncratic risk is reduced. Specifically, motivated by the results in Panel A of Table 8, which show the cross-sectional dispersion in the returns to be most strongly related to ME and ILLIQ, we calculate the conditional alphas associated with the ME and ILLIQ portfolio sorts. For the ME sorted portfolios, in particular, the spread in the average conditional alphas between the High and Low quintile sorted portfolios is reduced from $-1.40 \%$ for the standard conditional CAPM to $-0.99 \%$ for the generalized alphas, with a corresponding drop in the $t$-statistics from -4.73 to -3.21 . Thus, even though the size effect remains significant, part of it can be accounted for by the separate pricing of market diffusive and jump price risks. Similarly, for the ILLIQ sorted portfolios, the values of the conditional alphas for the HighLow quintile sorted portfolio is reduced from $1.16 \%$ to $0.77 \%$, with a corresponding drop in the $t$-statistics from 3.68 to 2.09, again underscoring the improvements in the cross-sectional pricing afforded by allowing for separate diffusive and jump risk premia. 


\subsection{Betas and macroeconomic news announcements}

An extensive literature has been devoted to studying the effects of macroeconomic news announcements on asset prices. Andersen et al. (2007a), Lee (2012), Lahaye et al. (2011), in particular, have all sought to relate jumps in high-frequency asset prices, with the significant jumps identified through similar techniques to the ones used here, to regularly scheduled macroeconomic news releases. Related to this, Savor and Wilson (2014) have also recently argued that cross-sectional return patterns might be different on news announcement days. ${ }^{42}$

To investigate whether macroeconomic announcement days confound our beta estimates and the significant cross-sectional relation between the two rough betas and expected stock returns, we exclude three specific announcement days in our estimation of $\beta^{c}$, $\beta^{d}$, and $\beta^{n}$, including days on which the employment report (Employment) and the Producer Price Index (PPI) are announced by the Bureau of Labor Statistics, and days when scheduled interest rates are announced by the Federal Open Market Committee (FOMC). Employment and PPI are both announced monthly at 8:30am before the stock market officially opens, while FOMC is announced at 2:15pm every six weeks. ${ }^{43}$

Relying on the same test for significant intraday jumps used above, Figure 5 compares the average jump intensity for the S\&P 500 market portfolio for the three announcement days and all other days in the sample (Non-Ann), as a function of the time-of-day. Not surprisingly, the FOMC announcements at 2:15pm have the greatest intraday effect, increasing the jump intensity from an average of about $1 \%$ per day on non-announcement days to $9 \%$ on FOMC days. ${ }^{44}$ The employment report also makes a market jump more likely in the first few minutes of trading, although not dramatically so. Of course, Employment and PPI are both announced before the market officially opens, and thus might be expected to affect estimation of the overnight betas the most.

Table 11 reports results of the full firm-level cross-sectional regressions excluding the three different types of announcement days. Both the size and the statistical significance of the risk premium estimates are very similar to those in Table 8, Panel B. In fact, the estimated risk premium for the discontinuous and overnight betas in Regression X in Table 11 is identical within two decimal points to the estimate from Regression XIV in Table 8. The predictive power and significant cross-sectional pricing ability of the two rough betas do not appear to be solely driven by important macroeconomic news announcements.

\footnotetext{
${ }^{42}$ Patton and Verardo (2012) also document that standard realized betas calculated from high-frequency intraday data tend to be higher on individual firms' earnings announcement days.

${ }^{43}$ Andersen et al. (2003) provide a comprehensive list of U.S. macroeconomic news announcements and their release times.

${ }^{44}$ Lucca and Moench (2014) have also recently documented large average pre-FOMC one-day equity returns in anticipation of monetary policy decisions.
} 


\section{Conclusion}

Based on a general continuous-time representation for the return on the aggregate market portfolio coupled with an economy-wide pricing kernel that separately prices market diffusive and jump risks, we show how standard asset pricing theory naturally results in separate risk premia for continuous, or smooth, market betas and discontinuous, or rough, market betas. Importantly, our theoretical framework explicitly allows for other systematic risk factors to enter the pricing kernel and possibly affect the cross-sectional pricing. Only if non-market risks are not priced, and the premia for continuous and jump market risks are the same, does the standard conditional CAPM hold.

Motivated by these theoretical results, we empirically investigate whether market diffusive and jump risks are priced differently in the cross-section of expected stock returns. Our empirical investigations rely on a unique high-frequency dataset for a large cross-section of individual stocks together with new econometric techniques for separately estimating continuous, discontinuous and overnight betas. We find that the discontinuous and overnight betas are different from, and more cross-sectionally dispersed than, the continuous and standard CAPM betas. When we sort individual stocks by the different betas, we find that stocks with high discontinuous and overnight betas earn significantly higher risk-adjusted returns than stocks with low discontinuous and overnight betas, while at best there is only a weak relation between a stock's return and its continuous beta. We also find the estimated risk premiums

for the discontinuous and overnight betas to be both statistically significant and indistinguishable from one another, and that this rough beta risk cannot be explained by a long list of other firm characteristics and explanatory variables commonly employed in the literature. By contrast, the estimated continuous beta risk premium is insignificant. Intuitively, market jumps and overnight price changes more likely reflect true information surprises than do continuous price moves, which may simply be attributed to random "noise" in the price formation process. Hence, the two rough betas more accurately reflect the systematic market price risks that are actually priced than do the continuous betas and the standard conditional CAPM betas that do not differentiate between continuous and discontinuous market price moves. 


\section{Data Appendix}

\section{A.1 High-frequency data cleaning}

We begin by removing entries that satisfy at least one criteria as follows: (i) a time stamp outside the exchange open window between 9:30am and 4:00pm; (ii) a price less than or equal to zero; (iii) a trade size less than or equal to zero; (iv) corrected trades, i.e., trades with Correction Indicator, CORR, other than 0, 1, or 2; and (v) an abnormal sale condition, i.e., trades for which the Sale Condition, COND, has a letter code other than '@', '*', 'E', 'F', '@E', '@F', ‘*E' and '*F'. We then assign a single value to each variable for each second within the 9:30am-4:00pm time interval as follows. If one or multiple transactions have occurred in that second, we calculate the sum of volumes, the sum of trades, and the volume-weighted average price within that second. If no transaction has occurred in that second, we enter zero for volume and trades. For the volume-weighted average price, we use the entry from the nearest previous second, i.e., forward-filtering. If no transaction has occurred before that second, we use the entry from the nearest subsequent second, i.e., backward-filtering. Motivated by our analysis of the trading volume distribution across different exchanges over time we purposely incorporate information from all exchanges covered by the TAQ database. Further details are provided in the supplementary data appendix Bollerslev et al. (2014).

\section{A.2 Additional explanatory variables}

Our empirical investigations rely on the following explanatory variables and firm characteristics.

- Size (ME): Following Fama and French (1993), a firm's size is measured at the end of June by its market value of equity - the product of the closing price and the number of shares outstanding (in millions of dollars). Market equity is updated annually and is used to explain returns over the subsequent 12 months. Following common practice, we also transform the size variable by its natural logarithm to reduce skewness.

- Book-to-market ratio (BM): Following Fama and French (1992), the book-to-market ratio in June of year $t$ is computed as the ratio of the book value of common equity in fiscal year $t-1$ to the market value of equity (size) in December of year $t-1 .{ }^{45}$ $\mathrm{BM}$ for fiscal year $t$ is used to explain returns from July of year $t+1$ through June of year $t+2$. The time gap between $\mathrm{BM}$ and returns ensures that information on $\mathrm{BM}$ is publicly available prior to the returns.

\footnotetext{
${ }^{45}$ Book common equity is defined as book value of stockholders' equity, plus balance-sheet deferred taxes and investment tax credit (if available), minus book value of preferred stock for fiscal year $t-1$.
} 
- Momentum (MOM): Following Jegadeesh and Titman (1993), the momentum variable at the end of month $t$ is defined as the compound gross return from month $t-11$ through month $t-1$; i.e., skipping the short-term reversal month $t .{ }^{46}$

- Reversal (REV): Following Jegadeesh (1990) and Lehmann (1990), the short-term reversal variable at the end of month $t$ is defined as the return over that same month $t$.

- Idiosyncratic volatility (IVOL): Following Ang et al. (2006b), a firm's idiosyncratic volatility at the end of month $t$ is computed as the standard deviation of the residuals from the regression based on the daily return regression:

$$
r_{i, d}-r_{f, d}=\alpha_{i}+\beta_{i}\left(r_{0, d}-r_{f, d}\right)+\gamma_{i} \mathrm{SMB}_{d}+\phi_{i} \mathrm{HML}_{d}+\epsilon_{i, d},
$$

where $r_{i, d}$ and $r_{0, d}$ are the daily returns of stock $i$ and the market portfolio on day $d$, respectively, and $\mathrm{SMB}_{d}$ and $\mathrm{HML}_{d}$ denote the daily Fama and French (1993) size and book-to-market factors.

- Coskewness (CSK): Following Harvey and Siddique (2000) and Ang et al. (2006a), the coskewness of stock $i$ at the end of month $t$ is estimated using daily returns for month $t$ as:

$$
\widehat{\mathrm{CSK}_{i, t}}=\frac{\frac{1}{N} \sum_{d}\left(r_{i, d}-\bar{r}_{i}\right)\left(r_{0, d}-\bar{r}_{0}\right)^{2}}{\sqrt{\frac{1}{N} \sum_{d}\left(r_{i, d}-\bar{r}_{i}\right)^{2}}\left(\frac{1}{N} \sum_{d}\left(r_{0, d}-\bar{r}_{0}\right)^{2}\right)},
$$

where $N$ denotes the number of trading days in month $t, r_{i, d}$ and $r_{0, d}$ are the daily returns of stock $i$ and the market portfolio on day $d$, respectively, and $\bar{r}_{i}$ and $\bar{r}_{0}$ denote the corresponding average daily returns.

- Cokurtosis (CKT): Following Ang et al. (2006a), the cokurtosis of stock $i$ at the end of month $t$ is estimated using the daily returns for month $t$ as:

$$
\widehat{\mathrm{CKT}_{i, t}}=\frac{\frac{1}{N} \sum_{d}\left(r_{i, d}-\bar{r}_{i}\right)\left(r_{0, d}-\bar{r}_{0}\right)^{3}}{\sqrt{\frac{1}{N} \sum_{d}\left(r_{i, d}-\bar{r}_{i}\right)^{2}}\left(\frac{1}{N} \sum_{d}\left(r_{0, d}-\bar{r}_{0}\right)^{2}\right)^{3 / 2}},
$$

where variables are the same as for CSK.

\footnotetext{
${ }^{46}$ Jegadeesh (1990) shows that monthly returns on many individual stocks are significantly and negatively serially correlated.
} 
- Realized skewness (RSK): Following Amaya et al. (2013), the realized skewness for stock $i$ on day $d$ is constructed from high-frequency data as:

$$
\operatorname{RSK}_{i, d}=\frac{\sqrt{L} \sum_{l=1}^{L} r_{i, d, l}^{3}}{\left(\sum_{l=1}^{L} r_{i, d, l}^{2}\right)^{3 / 2}}
$$

where $r_{i, d, l}$ refers to the $l$ th intraday return on day $d$ for stock $i$, and $L$ denotes the number of intraday returns available on day $d$. Consistent with Amaya et al. (2013), we use 5-minute returns from 9:45am to 4:00pm, so that for the full intraday time period $L=75$. The RSK for stock $i$ at the end of month $t$ is computed as the average of the daily $R S K_{i, d}$ for that month.

- Realized kurtosis (RKT): Following Amaya et al. (2013), the realized kurtosis for stock $i$ on day $d$ is computed as:

$$
\mathrm{RKT}_{i, d}=\frac{L \sum_{l=1}^{L} r_{i, d, l}^{4}}{\left(\sum_{l=1}^{L} r_{i, d, l}^{2}\right)^{2}}
$$

where variables and estimation details are the same as for RSK.

- Maximum daily return (MAX): Following Bali et al. (2011), the MAX variable for stock $i$ and month $t$ is defined as the largest total daily return observed over that month.

- Illiquidity (ILLIQ): Following Amihud (2002), the illiquidity for stock $i$ at the end of month $t$ is measured as the average daily ratio of the absolute stock return to the dollar trading volume from month $t-11$ through month $t$ :

$$
\mathrm{ILLIQ}_{i, t}=\frac{1}{N} \sum_{d}\left(\frac{\left|r_{i, d}\right|}{\text { volume }_{i, d} \times \text { price }_{i, d}}\right),
$$

where volume $e_{i, d}$ is the daily trading volume, price $_{i, d}$ is the daily price, and other variables are as defined before. We further transform the illiquidity measure by its natural logarithm to reduce skewness. 
Table 1: Cross-sectional relation of $\beta^{s}, \beta^{c}, \beta^{d}$, and $\beta^{n}$

The table reports the estimated regression coefficients, robust $t$-statistics (in parentheses) and adjusted $R^{2}$ s from Fama-MacBeth type regressions for explaining the cross-sectional variation in the standard $\beta^{s}$ as a function of the continuous beta $\beta^{c}$, the discontinuous beta $\beta^{d}$, and the overnight beta $\beta^{n}$. All of the betas are computed using 12-month returns.

\begin{tabular}{lllll}
\hline Regression & $\beta^{c}$ & $\beta^{d}$ & $\beta^{n}$ & Adjusted- $R^{2}$ \\
\hline I & 1.03 & & & 0.76 \\
& $(58.67)$ & & & 0.62 \\
II & & 0.79 & & 0.62 \\
& & $(26.72)$ & 0.51 & 0.46 \\
III & \multicolumn{5}{c}{$\begin{array}{l}(16.15) \\
\text { IV }\end{array}$} & 0.78 & 0.17 & 0.10 & 0.81 \\
& $(29.64)$ & $(6.87)$ & $(7.10)$ & \\
\hline
\end{tabular}


Table 2: Sample correlations

The table displays time-series averages of monthly cross-sectional correlations. The sample consists of the 985 individual stocks included in the S\&P 500 index over 1993-2010. $\beta^{s}, \beta^{c}, \beta^{d}$, and $\beta^{n}$ are the standard, continuous, discontinuous, and overnight betas, respectively. ME denotes the logarithm of the market capitalization of the firms. BM denotes the ratio of the book value of common equity to the market value of equity. MOM is the compound gross return from month $t-11$ through month $t-1$. REV is the month $t$ return. IVOL is a measure of idiosyncratic volatility. CSK and CKT are the measures of coskewness and cokurtosis, respectively. RSK and RKT denote the realized skewness and the realized kurtosis, respectively, computed from high-frequency data. MAX represents the maximum daily raw return for month $t$. ILLIQ refers to the logarithm of the average daily ratio of the absolute stock return to the dollar trading volume from month $t-11$ through month $t$. The asterisks indicate significance at the $5 \%(*)$ and $1 \%(* *)$ levels, respectively.

\begin{tabular}{|c|c|c|c|c|c|c|c|c|c|c|c|c|c|c|c|}
\hline & $\beta^{s}$ & $\beta^{c}$ & $\beta^{d}$ & $\beta^{n}$ & $\mathrm{ME}$ & $\mathrm{BM}$ & MOM & $\mathrm{REV}$ & IVOL & CSK & CKT & RSK & RKT & MAX & ILLIQ \\
\hline$\beta^{s}$ & 1 & $0.88^{* *}$ & $0.76^{* *}$ & $0.63^{* *}$ & $-0.12^{* *}$ & $-0.15^{* *}$ & $0.10^{* *}$ & 0.01 & $0.46^{* *}$ & $0.04^{*}$ & $0.38^{* *}$ & $-0.03^{* *}$ & $-0.07^{* *}$ & $0.47^{* *}$ & $-0.04^{*}$ \\
\hline$\beta^{c}$ & & 1 & $0.77^{* *}$ & $0.60^{* *}$ & -0.02 & $-0.17^{* *}$ & $0.09^{* *}$ & 0.01 & $0.43^{* *}$ & $0.07^{* *}$ & $0.26^{* *}$ & $-0.03^{* *}$ & $-0.15^{* *}$ & $0.43^{* *}$ & $-0.08^{* *}$ \\
\hline$\beta^{d}$ & & & 1 & $0.74^{* *}$ & $-0.27^{* *}$ & $-0.11^{* *}$ & $0.08^{* *}$ & 0.01 & $0.58^{* *}$ & 0.01 & $0.06^{* *}$ & $-0.05^{* *}$ & $-0.02^{*}$ & $0.53^{* *}$ & $0.15^{* *}$ \\
\hline$\beta^{n}$ & & & & 1 & $-0.22^{* *}$ & $-0.11^{* *}$ & $0.03^{* *}$ & 0.01 & $0.53^{* *}$ & 0.01 & -0.01 & $-0.04^{* *}$ & -0.02 & $0.48^{* *}$ & $0.13^{* *}$ \\
\hline MF & & & & & 1 & $-0.15^{* *}$ & $-0.04^{* *}$ & $-0.03^{* *}$ & $-0.34^{* *}$ & $0.04^{* *}$ & $0.30^{* *}$ & 0.00 & $-0.40^{* *}$ & $-0.28^{* *}$ & $-0.91^{* *}$ \\
\hline $\mathrm{BN}$ & & & & & & 1 & -0.04 & 0.00 & $-0.08^{* *}$ & $-0.06^{* *}$ & $-0.07^{* *}$ & 0.01 & $0.05^{* *}$ & $-0.07^{* *}$ & $0.14^{* *}$ \\
\hline $\mathrm{MC}$ & & & & & & & 1 & $0.02^{*}$ & 0.00 & $-0.07^{* *}$ & $0.06^{*}$ & -0.02 & $0.04^{* *}$ & 0.00 & $0.05^{* *}$ \\
\hline $\mathrm{RE}$ & & & & & & & & 1 & $0.03^{*}$ & -0.01 & $-0.02^{* *}$ & $0.37^{* *}$ & $0.02^{* *}$ & $0.30^{* *}$ & $-0.01^{*}$ \\
\hline IV & & & & & & & & & 1 & 0.00 & $-0.22^{* *}$ & $-0.04^{* *}$ & $0.11^{* *}$ & $0.81^{* *}$ & $0.26^{* *}$ \\
\hline CS & & & & & & & & & & 1 & -0.03 & $0.02^{* *}$ & $-0.05^{* *}$ & $0.03^{*}$ & $-0.05^{* *}$ \\
\hline CK & & & & & & & & & & & 1 & 0.00 & $-0.16^{* *}$ & $-0.11^{* *}$ & $-0.27^{* *}$ \\
\hline $\mathrm{RS}$ & & & & & & & & & & & & 1 & $0.04^{* *}$ & $0.04^{* *}$ & 0.00 \\
\hline RK & & & & & & & & & & & & & 1 & $0.07^{* *}$ & $0.41^{* *}$ \\
\hline $\mathrm{M} A$ & & & & & & & & & & & & & & 1 & $0.21^{* *}$ \\
\hline ILI & IQ & & & & & & & & & & & & & & 1 \\
\hline
\end{tabular}


Table 3: Portfolio characteristics sorted by betas

The table displays time-series averages of equal-weighted characteristics of stocks sorted by the four different betas. The sample consists of the 985 individual stocks included in the S\&P 500 index over 1993-2010. $\beta^{s}, \beta^{c}, \beta^{d}$, and $\beta^{n}$ are the standard, continuous, discontinuous, and overnight betas, respectively. ME denotes the logarithm of the market capitalization of firms. $\mathrm{BM}$ denotes the ratio of the book value of common equity to the market value of equity. MOM is the compound gross return from month $t-11$ through month $t-1$. REV is the month $t$ return. IVOL is a measure of idiosyncratic volatility. CSK and CKT are the measures of coskewness and cokurtosis, respectively. RSK and RKT denote the realized skewness and the realized kurtosis computed from high-frequency data. MAX represents the maximum daily raw return over month $t$. ILLIQ refers to the logarithm of the average daily ratio of the absolute stock return to the dollar trading volume from month $t-11$ through month $t$. Panel A displays the results sorted by $\beta^{s}$, Panel B by $\beta^{c}$, Panel C by $\beta^{d}$, and Panel D by $\beta^{n}$.

\begin{tabular}{|c|c|c|c|c|c|c|c|c|c|c|c|c|c|c|c|}
\hline & $\beta^{s}$ & $\beta^{c}$ & $\beta^{d}$ & $\beta^{n}$ & $\mathrm{ME}$ & $\mathrm{BM}$ & MOM & $\mathrm{REV}$ & IVOL & CSK & CKT & RSK & RKT & MAX & ILLIQ \\
\hline \multicolumn{16}{|c|}{ Panel A: Sorted by $\beta^{s}$} \\
\hline 1(Low) & 0.45 & 0.52 & 1.02 & 1.11 & 8.48 & 0.56 & 8.37 & 0.76 & 1.37 & -0.10 & 1.51 & 0.03 & 5.31 & 3.54 & -2.78 \\
\hline 2 & 0.73 & 0.70 & 1.12 & 1.22 & 8.56 & 0.54 & 9.59 & 0.86 & 1.48 & -0.10 & 2.04 & 0.04 & 5.42 & 4.03 & -3.08 \\
\hline 3 & 0.93 & 0.83 & 1.23 & 1.37 & 8.58 & 0.47 & 10.59 & 0.95 & 1.60 & -0.10 & 2.31 & 0.03 & 5.39 & 4.52 & -3.18 \\
\hline 4 & 1.18 & 1.03 & 1.45 & 1.67 & 8.62 & 0.50 & 12.80 & 1.26 & 1.83 & -0.09 & 2.48 & 0.02 & 5.26 & 5.33 & -3.28 \\
\hline 5(High) & 1.83 & 1.58 & 2.08 & 2.43 & 8.43 & 0.43 & 13.84 & 1.48 & 2.57 & -0.09 & 2.52 & 0.02 & 5.10 & 7.78 & -3.40 \\
\hline \multicolumn{16}{|c|}{ Panel B: Sorted by $\beta^{c}$} \\
\hline 1(Low) & 0.57 & 0.44 & 0.99 & 1.14 & 8.54 & 0.54 & 7.80 & 0.81 & 1.39 & -0.11 & 1.73 & 0.03 & 5.54 & 3.68 & -2.32 \\
\hline 2 & 0.79 & 0.67 & 1.09 & 1.22 & 8.73 & 0.50 & 11.28 & 0.83 & 1.47 & -0.11 & 2.12 & 0.03 & 5.40 & 4.07 & -2.86 \\
\hline 3 & 0.95 & 0.83 & 1.23 & 1.38 & 8.77 & 0.45 & 10.55 & 0.95 & 1.59 & -0.10 & 2.31 & 0.03 & 5.31 & 4.53 & -3.29 \\
\hline 4 & 1.18 & 1.05 & 1.46 & 1.67 & 8.86 & 0.49 & 11.40 & 1.13 & 1.83 & -0.10 & 2.45 & 0.03 & 5.13 & 5.29 & -3.52 \\
\hline 5(High) & 1.80 & 1.65 & 2.15 & 2.45 & 8.63 & 0.41 & 12.60 & 1.44 & 2.54 & -0.08 & 2.44 & 0.02 & 4.94 & 7.64 & -3.57 \\
\hline \multicolumn{16}{|c|}{ Panel C: Sorted by $\beta^{d}$} \\
\hline 1(Low) & 0.60 & 0.53 & 0.80 & 0.93 & 8.94 & 0.54 & 9.16 & 0.78 & 1.15 & -0.10 & 1.96 & 0.04 & 5.46 & 3.14 & -3.35 \\
\hline 2 & 0.81 & 0.71 & 1.03 & 1.17 & 8.91 & 0.49 & 9.68 & 0.83 & 1.39 & -0.10 & 2.22 & 0.04 & 5.32 & 3.90 & -3.23 \\
\hline 3 & 0.96 & 0.85 & 1.22 & 1.40 & 8.86 & 0.49 & 10.62 & 0.90 & 1.60 & -0.10 & 2.28 & 0.03 & 5.22 & 4.54 & -3.26 \\
\hline 4 & 1.18 & 1.03 & 1.50 & 1.72 & 8.68 & 0.49 & 11.24 & 1.09 & 1.92 & -0.09 & 2.32 & 0.03 & 5.16 & 5.51 & -3.19 \\
\hline 5(High) & 1.73 & 1.51 & 2.37 & 2.63 & 8.14 & 0.37 & 13.33 & 1.56 & 2.77 & -0.09 & 2.27 & 0.02 & 5.16 & 8.16 & -2.82 \\
\hline \multicolumn{16}{|c|}{ Panel D: Sorted by $\beta^{n}$} \\
\hline 1(Low) & 0.64 & 0.59 & 0.90 & 0.78 & 8.95 & 0.55 & 9.48 & 0.71 & 1.14 & -0.10 & 2.06 & 0.04 & 5.38 & 3.11 & -3.56 \\
\hline 2 & 0.83 & 0.74 & 1.09 & 1.08 & 8.91 & 0.50 & 10.11 & 0.81 & 1.41 & -0.10 & 2.24 & 0.04 & 5.30 & 3.93 & -3.27 \\
\hline 3 & 0.98 & 0.86 & 1.26 & 1.34 & 8.81 & 0.49 & 10.83 & 0.93 & 1.61 & -0.10 & 2.27 & 0.03 & 5.26 & 4.58 & -3.23 \\
\hline 4 & 1.20 & 1.04 & 1.50 & 1.72 & 8.66 & 0.48 & 11.83 & 1.12 & 1.93 & -0.10 & 2.32 & 0.02 & 5.18 & 5.56 & -3.20 \\
\hline 5(High) & 1.65 & 1.42 & 2.17 & 2.95 & 8.20 & 0.35 & 14.51 & 1.60 & 2.75 & -0.09 & 2.17 & 0.02 & 5.19 & 8.09 & -2.97 \\
\hline
\end{tabular}


Table 4: Equal-weighted returns of contemporaneous single-sorted portfolios

The table reports equal-weighted average returns and betas for contemporaneous single-sorted portfolios. The sample consists of the 985 individual stocks included in the S\&P 500 index over 1993-2010. At the beginning of each month, stocks are sorted into quintiles according to betas computed from the next 12-month returns. Each equal-weighted portfolio is held for 12 months. The column labeled "Return" reports the average monthly excess return in the 12-month holding period for each portfolio. The row labeled "High-Low" reports the difference in returns between portfolio 5 and portfolio 1, with Newey-West robust $t$-statistics in parentheses. $\beta^{s}, \beta^{c}, \beta^{d}$, and $\beta^{n}$ are the standard, continuous, discontinuous, and overnight betas, respectively. Panel A displays the results sorted by $\beta^{s}$, Panel B by $\beta^{c}$, Panel C by $\beta^{d}$, Panel D by $\beta^{n}$, Panel E by $\beta^{d}-\beta^{s}$, and Panel F by $\beta^{n}-\beta^{s}$.

Panel A: Sorted by $\beta^{s}$

\begin{tabular}{|c|c|c|c|c|c|}
\hline Decile & $\beta^{s}$ & $\beta^{c}$ & $\beta^{d}$ & $\beta^{n}$ & Return \\
\hline 1(Low) & 0.45 & 0.52 & 1.02 & 1.11 & $\begin{array}{c}0.77 \\
(3.66)\end{array}$ \\
\hline 2 & 0.73 & 0.70 & 1.12 & 1.22 & $\begin{array}{c}0.81 \\
(3.14)\end{array}$ \\
\hline 3 & 0.93 & 0.83 & 1.23 & 1.37 & $\begin{array}{c}0.97 \\
(3.21)\end{array}$ \\
\hline 4 & 1.18 & 1.03 & 1.45 & 1.67 & $\begin{array}{c}1.17 \\
(3.18)\end{array}$ \\
\hline 5(High) & 1.83 & 1.58 & 2.08 & 2.43 & $\begin{array}{c}2.39 \\
(2.90)\end{array}$ \\
\hline High-Low & 1.38 & 1.06 & 1.06 & 1.32 & $\begin{array}{c}1.62 \\
(1.85)\end{array}$ \\
\hline
\end{tabular}

Panel C: Sorted by $\beta^{d}$

\begin{tabular}{|c|c|c|c|c|c|}
\hline Decile & $\beta^{s}$ & $\beta^{c}$ & $\beta^{d}$ & $\beta^{n}$ & Return \\
\hline 1 (Low) & 0.60 & 0.53 & 0.80 & 0.93 & $\begin{array}{c}0.78 \\
(3.80)\end{array}$ \\
\hline 2 & 0.81 & 0.71 & 1.03 & 1.17 & $\begin{array}{c}0.83 \\
(3.21)\end{array}$ \\
\hline 3 & 0.96 & 0.85 & 1.22 & 1.40 & $\begin{array}{c}0.85 \\
(2.99)\end{array}$ \\
\hline 4 & 1.18 & 1.03 & 1.50 & 1.72 & $\begin{array}{c}1.21 \\
(3.31)\end{array}$ \\
\hline 5(High) & 1.73 & 1.51 & 2.37 & 2.63 & $\begin{array}{c}2.48 \\
(2.97)\end{array}$ \\
\hline High-Low & 1.13 & 0.98 & 1.57 & 1.70 & $\begin{array}{c}1.71 \\
(2.63)\end{array}$ \\
\hline
\end{tabular}

Panel E: Sorted by $\beta^{d}-\beta^{s}$

\begin{tabular}{|c|c|c|c|c|c|}
\hline Decile & $\beta^{s}$ & $\beta^{c}$ & $\beta^{d}$ & $\beta^{n}$ & Return \\
\hline 1(Low) & 1.17 & 0.96 & 1.22 & 1.43 & $\begin{array}{c}0.97 \\
(2.68)\end{array}$ \\
\hline 2 & 0.96 & 0.87 & 1.13 & 1.30 & $\begin{array}{c}0.94 \\
(3.44)\end{array}$ \\
\hline 3 & 0.95 & 0.84 & 1.20 & 1.37 & $\begin{array}{c}1.08 \\
(3.88)\end{array}$ \\
\hline 4 & 1.02 & 0.87 & 1.36 & 1.55 & $\begin{array}{c}1.25 \\
(4.13)\end{array}$ \\
\hline 5(High) & 1.19 & 1.10 & 2.02 & 2.22 & $\begin{array}{c}1.91 \\
(3.56)\end{array}$ \\
\hline High-Low & 0.02 & 0.14 & 0.80 & 0.79 & 0.94 \\
\hline
\end{tabular}

Panel B: Sorted by $\beta^{c}$

\begin{tabular}{cccccc} 
Decile & $\beta^{s}$ & $\beta^{c}$ & $\beta^{d}$ & $\beta^{n}$ & Return \\
\hline 1 (Low) & 0.57 & 0.44 & 0.99 & 1.14 & 0.80 \\
& & & & & $(3.55)$ \\
2 & 0.79 & 0.67 & 1.09 & 1.22 & 0.82 \\
& & & & & $(3.14)$ \\
3 & 0.95 & 0.83 & 1.23 & 1.38 & 0.91 \\
& & & & & $(3.15)$ \\
4 & 1.18 & 1.05 & 1.46 & 1.67 & 1.20 \\
& & & & & $\begin{array}{c}3.26) \\
5 \text { (High) }\end{array}$ \\
& 1.80 & 1.65 & 2.15 & 2.45 & $\left.\begin{array}{c}2.41 \\
2.92\end{array}\right)$ \\
High-Low & 1.23 & 1.21 & 1.16 & 1.31 & 1.61 \\
& & & & & $(1.81)$
\end{tabular}

Panel D: Sorted by $\beta^{n}$

\begin{tabular}{|c|c|c|c|c|c|}
\hline Decile & $\beta^{s}$ & $\beta^{c}$ & $\beta^{d}$ & $\beta^{n}$ & Return \\
\hline 1(Low) & 0.64 & 0.59 & 0.90 & 0.78 & $\begin{array}{c}0.75 \\
(3.84)\end{array}$ \\
\hline 2 & 0.83 & 0.74 & 1.09 & 1.08 & $\begin{array}{c}0.83 \\
(3.37)\end{array}$ \\
\hline 3 & 0.98 & 0.86 & 1.26 & 1.34 & $\begin{array}{c}0.96 \\
(3.29)\end{array}$ \\
\hline 4 & 1.20 & 1.04 & 1.50 & 1.72 & $\begin{array}{c}1.22 \\
(3.29)\end{array}$ \\
\hline 5(High) & 1.65 & 1.42 & 2.17 & 2.95 & $\begin{array}{c}2.39 \\
(2.91)\end{array}$ \\
\hline High-Low & 1.01 & 0.83 & 1.27 & 2.17 & $\begin{array}{c}1.64 \\
(2.59)\end{array}$ \\
\hline
\end{tabular}

Panel F: Sorted by $\beta^{n}-\beta^{s}$

\begin{tabular}{|c|c|c|c|c|c|}
\hline Decile & $\beta^{s}$ & $\beta^{c}$ & $\beta^{d}$ & $\beta^{n}$ & Return \\
\hline 1(Low) & 1.16 & 1.03 & 1.29 & 1.06 & $\begin{array}{c}0.98 \\
(3.22)\end{array}$ \\
\hline 2 & 0.93 & 0.84 & 1.17 & 1.13 & $\begin{array}{c}1.17 \\
(3.87)\end{array}$ \\
\hline 3 & 0.97 & 0.86 & 1.24 & 1.33 & $\begin{array}{c}1.17 \\
(4.16)\end{array}$ \\
\hline 4 & 1.07 & 0.91 & 1.38 & 1.62 & $\begin{array}{c}1.29 \\
(3.80)\end{array}$ \\
\hline 5(High) & 1.15 & 1.00 & 1.85 & 2.73 & $\begin{array}{c}1.68 \\
(3.01)\end{array}$ \\
\hline High-Low & -0.01 & -0.03 & 0.56 & 1.67 & $\begin{array}{c}0.69 \\
(3.18)\end{array}$ \\
\hline
\end{tabular}


Table 5: Value-weighted returns of contemporaneous single-sorted portfolios

The table reports value-weighted average returns and betas for contemporaneous single-sorted portfolios. The sample consists of the 985 individual stocks included in the S\&P 500 index over 1993-2010. At the beginning of each month, stocks are sorted into quintiles according to betas computed from the next 12-month returns. Each value-weighted portfolio is held for 12 months. The column labeled "Return" reports the average monthly excess return in the 12-month holding period for each portfolio. The row labeled "High-Low" reports the difference in returns between portfolio 5 and portfolio 1, with Newey-West robust $t$-statistics in parentheses. $\beta^{s}, \beta^{c}, \beta^{d}$, and $\beta^{n}$ are the standard, continuous, discontinuous, and overnight betas, respectively. Panel A displays the results sorted by $\beta^{s}$, Panel B by $\beta^{c}$, Panel C by $\beta^{d}$, Panel D by $\beta^{n}$, Panel E by $\beta^{d}-\beta^{s}$, and Panel F by $\beta^{n}-\beta^{s}$.

Panel A: Sorted by $\beta^{s}$

\begin{tabular}{|c|c|c|c|c|c|}
\hline Decile & $\beta^{s}$ & $\beta^{c}$ & $\beta^{d}$ & $\beta^{n}$ & Return \\
\hline 1(Low) & 0.47 & 0.57 & 0.91 & 0.98 & $\begin{array}{c}0.62 \\
(3.75)\end{array}$ \\
\hline 2 & 0.73 & 0.75 & 1.07 & 1.12 & $\begin{array}{c}0.63 \\
(2.62)\end{array}$ \\
\hline 3 & 0.93 & 0.89 & 1.19 & 1.29 & $\begin{array}{c}0.85 \\
(3.10)\end{array}$ \\
\hline 4 & 1.19 & 1.10 & 1.39 & 1.57 & $\begin{array}{c}0.91 \\
(2.68)\end{array}$ \\
\hline 5(High) & 1.74 & 1.55 & 1.85 & 2.18 & $\begin{array}{c}1.71 \\
(2.53)\end{array}$ \\
\hline High-Low & 1.27 & 0.98 & 0.95 & 1.21 & $\begin{array}{c}1.10 \\
(1.59)\end{array}$ \\
\hline
\end{tabular}

Panel C: Sorted by $\beta^{d}$

\begin{tabular}{|c|c|c|c|c|c|}
\hline Decile & $\beta^{s}$ & $\beta^{c}$ & $\beta^{d}$ & $\beta^{n}$ & Return \\
\hline 1(Low) & 0.60 & 0.59 & 0.81 & 0.90 & $\begin{array}{c}0.68 \\
(3.68)\end{array}$ \\
\hline 2 & 0.81 & 0.78 & 1.03 & 1.15 & $\begin{array}{c}0.74 \\
(3.02)\end{array}$ \\
\hline 3 & 1.01 & 0.94 & 1.22 & 1.35 & $\begin{array}{c}0.87 \\
(2.98)\end{array}$ \\
\hline 4 & 1.27 & 1.16 & 1.50 & 1.68 & $\begin{array}{c}1.17 \\
(2.94)\end{array}$ \\
\hline 5(High) & 1.74 & 1.59 & 2.21 & 2.41 & $\begin{array}{c}2.23 \\
(2.60)\end{array}$ \\
\hline High-Low & 1.14 & 1.00 & 1.40 & 1.51 & $\begin{array}{c}1.56 \\
(2.41)\end{array}$ \\
\hline
\end{tabular}

Panel E: Sorted by $\beta^{d}-\beta^{s}$

\begin{tabular}{|c|c|c|c|c|c|}
\hline Decile & $\beta^{s}$ & $\beta^{c}$ & $\beta^{d}$ & $\beta^{n}$ & Return \\
\hline 1(Low) & 1.18 & 1.05 & 1.19 & 1.37 & $\begin{array}{c}0.94 \\
(2.37)\end{array}$ \\
\hline 2 & 0.95 & 0.90 & 1.15 & 1.27 & $\begin{array}{c}0.96 \\
(3.06)\end{array}$ \\
\hline 3 & 0.95 & 0.87 & 1.22 & 1.36 & $\begin{array}{c}1.03 \\
(3.59)\end{array}$ \\
\hline 4 & 1.01 & 0.90 & 1.39 & 1.52 & $\begin{array}{c}1.23 \\
(3.86)\end{array}$ \\
\hline 5(High) & 1.12 & 1.02 & 1.97 & 2.08 & $\begin{array}{c}1.74 \\
(2.99)\end{array}$ \\
\hline High-Low & -0.06 & -0.03 & 0.78 & 0.71 & $\begin{array}{c}0.80 \\
(2.43)\end{array}$ \\
\hline
\end{tabular}

Panel B: Sorted by $\beta^{c}$

\begin{tabular}{|c|c|c|c|c|c|}
\hline Decile & $\beta^{s}$ & $\beta^{c}$ & $\beta^{d}$ & $\beta^{n}$ & Return \\
\hline 1 (Low) & 0.53 & 0.46 & 0.87 & 0.99 & $\begin{array}{c}0.72 \\
(3.90)\end{array}$ \\
\hline 2 & 0.72 & 0.67 & 1.00 & 1.07 & $\begin{array}{c}0.65 \\
(2.85)\end{array}$ \\
\hline 3 & 0.88 & 0.83 & 1.13 & 1.22 & $\begin{array}{c}0.68 \\
(2.59)\end{array}$ \\
\hline 4 & 1.13 & 1.05 & 1.34 & 1.50 & $\begin{array}{c}0.88 \\
(2.67)\end{array}$ \\
\hline 5(High) & 1.65 & 1.55 & 1.84 & 2.11 & $\begin{array}{c}1.69 \\
(2.57)\end{array}$ \\
\hline High-Low & 1.12 & 1.09 & 0.97 & 1.12 & $\begin{array}{c}0.98 \\
(1.58)\end{array}$ \\
\hline
\end{tabular}

Panel D: Sorted by $\beta^{n}$

\begin{tabular}{|c|c|c|c|c|c|}
\hline Decile & $\beta^{s}$ & $\beta^{c}$ & $\beta^{d}$ & $\beta^{n}$ & Return \\
\hline 1(Low) & 0.63 & 0.64 & 0.91 & 0.78 & $\begin{array}{c}0.65 \\
(3.90)\end{array}$ \\
\hline 2 & 0.84 & 0.81 & 1.09 & 1.07 & $\begin{array}{c}0.80 \\
(3.44)\end{array}$ \\
\hline 3 & 1.01 & 0.95 & 1.24 & 1.34 & $\begin{array}{c}1.01 \\
(3.48)\end{array}$ \\
\hline 4 & 1.25 & 1.14 & 1.46 & 1.71 & $\begin{array}{c}1.13 \\
(2.83)\end{array}$ \\
\hline 5(High) & 1.62 & 1.45 & 1.93 & 2.75 & $\begin{array}{c}2.26 \\
(2.43)\end{array}$ \\
\hline High-Low & 0.99 & 0.81 & 1.02 & 1.97 & $\begin{array}{c}1.60 \\
(2.48)\end{array}$ \\
\hline
\end{tabular}

Panel F: Sorted by $\beta^{n}-\beta^{s}$

\begin{tabular}{|c|c|c|c|c|c|}
\hline Decile & $\beta^{s}$ & $\beta^{c}$ & $\beta^{d}$ & $\beta^{n}$ & Return \\
\hline 1(Low) & 1.13 & 1.03 & 1.23 & 1.05 & $\begin{array}{c}0.96 \\
(3.09)\end{array}$ \\
\hline 2 & 0.93 & 0.88 & 1.15 & 1.16 & $\begin{array}{c}1.01 \\
(3.71)\end{array}$ \\
\hline 3 & 0.98 & 0.90 & 1.22 & 1.37 & $\begin{array}{c}1.12 \\
(3.60)\end{array}$ \\
\hline 4 & 1.10 & 0.97 & 1.35 & 1.68 & $\begin{array}{c}1.16 \\
(2.60)\end{array}$ \\
\hline 5(High) & 1.15 & 1.06 & 1.65 & 2.66 & $\begin{array}{c}1.46 \\
(2.19)\end{array}$ \\
\hline High-Low & 0.02 & 0.03 & 0.41 & 1.61 & $\begin{array}{c}0.49 \\
(2.52)\end{array}$ \\
\hline
\end{tabular}


Table 6: Average returns of predictive single-sorted portfolios

The table reports equal-weighted average returns and betas for predictive single-sorted portfolios. The sample consists of the 985 individual stocks included in the S\&P 500 index over 1993-2010. At the end of each month, stocks are sorted into Quintiles according to betas computed from previous 12-month returns. Each portfolio is held for one month. The column labeled "Ex-Post" reports the ex-post betas compute from the subsequent 12-month returns. The column labeled "Return" reports the average one-month ahead excess returns of each portfolio. The column labeled "FFC4 alpha" reports the corresponding Fama-French-Carhart four-factor alpha for each portfolio. The row labeled "High-Low" reports the difference in returns between portfolio 10 and portfolio 1 , with Newey-West robust $t$-statistics in parentheses. $\beta^{s}, \beta^{c}, \beta^{d}$, and $\beta^{n}$ are the standard, continuous, discontinuous, and overnight betas, respectively. Panel A displays the results sorted by $\beta^{s}$, Panel $\mathrm{B}$ by $\beta^{c}$, Panel $\mathrm{C}$ by $\beta^{d}$, Panel D by $\beta^{n}$, and Panel E by $\beta^{s}-\beta^{c}$.

Panel A: Sorted by $\beta^{s}$

\begin{tabular}{ccccccccc} 
Quintile & $\beta^{s}$ & $\beta^{c}$ & $\beta^{d}$ & $\beta^{n}$ & $\beta^{s}$ & Return & alpha \\
\hline $1($ Low $)$ & 0.45 & 0.52 & 1.02 & 1.11 & 0.60 & 0.76 & 0.32 \\
& & & & & & $(3.09)$ & $(2.07)$ \\
2.00 & 0.73 & 0.70 & 1.12 & 1.22 & 0.80 & 0.85 & 0.31 \\
& & & & & & $(2.74)$ & $(2.08)$ \\
3.00 & 0.93 & 0.83 & 1.23 & 1.37 & 0.95 & 1.00 & 0.38 \\
& & & & & & $(2.76)$ & $(2.95)$ \\
4.00 & 1.18 & 1.03 & 1.45 & 1.67 & 1.12 & 1.23 & 0.57 \\
& & & & & & $(2.86)$ & $(4.12)$ \\
5 (High) & 1.83 & 1.58 & 2.08 & 2.43 & 1.63 & 1.59 & 0.90 \\
& & & & & & & $(2.29)$ & $(3.87)$ \\
High-Low & 1.38 & 1.06 & 1.06 & 1.32 & 1.04 & 0.83 & 0.58 \\
& & & & & & $(1.40)$ & $(1.76)$
\end{tabular}

Panel C: Sorted by $\beta^{d}$

\begin{tabular}{|c|c|c|c|c|c|c|c|}
\hline Quintile & $\beta^{s}$ & $\beta^{c}$ & $\beta^{d}$ & $\beta^{n}$ & $\begin{array}{c}\text { Ex-Post } \\
\beta^{d}\end{array}$ & Return & $\begin{array}{l}\text { FFC4 } \\
\text { alpha }\end{array}$ \\
\hline 1 (Low) & 0.60 & 0.53 & 0.80 & 0.93 & 0.93 & $\begin{array}{c}0.72 \\
(2.94)\end{array}$ & $\begin{array}{c}0.30 \\
(2.20)\end{array}$ \\
\hline 2 & 0.81 & 0.71 & 1.03 & 1.17 & 1.12 & $\begin{array}{c}0.76 \\
(2.37)\end{array}$ & $\begin{array}{c}0.23 \\
(1.73)\end{array}$ \\
\hline 3 & 0.96 & 0.85 & 1.22 & 1.40 & 1.24 & $\begin{array}{c}0.95 \\
(2.62)\end{array}$ & $\begin{array}{c}0.36 \\
(2.77)\end{array}$ \\
\hline 4 & 1.18 & 1.03 & 1.50 & 1.72 & 1.46 & $\begin{array}{c}1.17 \\
(2.72)\end{array}$ & $\begin{array}{c}0.52 \\
(3.71)\end{array}$ \\
\hline 5(High) & 1.73 & 1.51 & 2.37 & 2.63 & 2.07 & $\begin{array}{c}1.65 \\
(2.39)\end{array}$ & $\begin{array}{c}0.94 \\
(4.08)\end{array}$ \\
\hline High-Low & 1.13 & 0.98 & 1.57 & 1.70 & 1.13 & $\begin{array}{c}0.93 \\
(1.55)\end{array}$ & $\begin{array}{c}0.64 \\
(2.04)\end{array}$ \\
\hline
\end{tabular}

Panel E: Sorted by $\beta^{d}-\beta^{s}$

Ex-Post $\quad$ FFC4 Quintile $\beta^{s} \quad \beta^{c} \quad \beta^{d} \beta^{n} \beta^{d}-\beta^{s}$ Return alpha

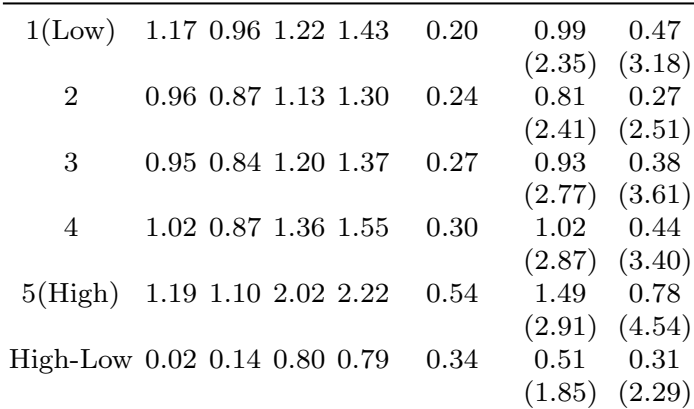

Panel B: Sorted by $\beta^{c}$

\begin{tabular}{|c|c|c|c|c|c|c|c|}
\hline Quintile & $\beta^{s}$ & $\beta^{c}$ & $\beta^{d}$ & $\beta^{n}$ & $\begin{array}{c}\text { Ex-Post } \\
\beta^{c}\end{array}$ & Return & $\begin{array}{l}\text { FFC4 } \\
\text { alpha }\end{array}$ \\
\hline 1 (Low) & 0.57 & 0.44 & 0.99 & 1.14 & 0.57 & $\begin{array}{c}0.79 \\
(3.05)\end{array}$ & $\begin{array}{c}0.33 \\
(2.20)\end{array}$ \\
\hline 2 & 0.79 & 0.67 & 1.09 & 1.22 & 0.74 & $\begin{array}{c}0.79 \\
(2.55)\end{array}$ & $\begin{array}{c}0.29 \\
(1.97)\end{array}$ \\
\hline 3 & 0.95 & 0.83 & 1.23 & 1.38 & 0.84 & $\begin{array}{c}0.97 \\
(2.65)\end{array}$ & $\begin{array}{c}0.35 \\
(2.59)\end{array}$ \\
\hline 4 & 1.18 & 1.05 & 1.46 & 1.67 & 1.02 & $\begin{array}{c}1.19 \\
(2.80)\end{array}$ & $\begin{array}{c}0.54 \\
(4.01)\end{array}$ \\
\hline 5(High) & 1.80 & 1.65 & 2.15 & 2.45 & 1.51 & $\begin{array}{c}1.50 \\
(2.18)\end{array}$ & $\begin{array}{c}0.82 \\
(3.45)\end{array}$ \\
\hline High-Low & 1.23 & 1.21 & 1.16 & 1.31 & 0.93 & $\begin{array}{c}0.71 \\
(1.22)\end{array}$ & $\begin{array}{c}0.49 \\
(1.44)\end{array}$ \\
\hline
\end{tabular}

Panel D: Sorted by $\beta^{n}$

\begin{tabular}{|c|c|c|c|c|c|c|c|}
\hline Quinti & $\beta^{s}$ & $\beta^{c}$ & $\beta^{d}$ & $\beta^{n}$ & $\begin{array}{c}\text { Ex-Post } \\
\beta^{n}\end{array}$ & Return & $\begin{array}{l}\text { FFC4 } \\
\text { alpha }\end{array}$ \\
\hline$(\mathrm{I}$ & 4 & 9 & 0.90 & 0 & 1 & & \\
\hline 2 & 83 & 4 & 1.09 & 1. & 10 & & \\
\hline 3 & 3 & 6 & 1.26 & 1. & 1. & & \\
\hline 4 & 20 & & 0 & 1. & 1.72 & & $\begin{array}{c}0.47 \\
(3.58)\end{array}$ \\
\hline $5(\mathrm{H}$ & 1.65 & 1.42 & 2.17 & 2.95 & 2.3 & $\begin{array}{c}1.75 \\
(2.60)\end{array}$ & $\begin{array}{c}1.06 \\
(4.69)\end{array}$ \\
\hline High-Low & 1.01 & 0.83 & 1.27 & 2.17 & 1.32 & $\begin{array}{c}1.11 \\
(1.93)\end{array}$ & $\begin{array}{c}0.85 \\
(2.74)\end{array}$ \\
\hline
\end{tabular}

Panel F: Sorted by $\beta^{n}-\beta^{s}$

\begin{tabular}{|c|c|c|c|c|c|c|c|}
\hline Quintile & $\beta^{s}$ & $\beta^{c}$ & $\beta^{d}$ & $\beta^{n}$ & $\begin{array}{l}\text { Ex-Post } \\
\beta^{n}-\beta^{s}\end{array}$ & Return & $\begin{array}{l}\text { FFC4 } \\
\text { alpha }\end{array}$ \\
\hline 1 (Low) & 1.16 & 0.98 & 1.29 & 1.06 & 0.31 & $\begin{array}{c}0.68 \\
(1.80)\end{array}$ & $\begin{array}{c}0.15 \\
(0.82)\end{array}$ \\
\hline 2 & 0.93 & 0.84 & 1.17 & 1.13 & 0.38 & $\begin{array}{c}0.81 \\
(2.40)\end{array}$ & $\begin{array}{c}0.23 \\
(1.91)\end{array}$ \\
\hline 3 & 0.97 & 0.86 & 1.24 & 1.33 & 0.47 & $\begin{array}{c}1.01 \\
(2.99)\end{array}$ & $\begin{array}{c}0.43 \\
(3.74)\end{array}$ \\
\hline 4 & 1.07 & 0.91 & 1.38 & 1.62 & 0.57 & $\begin{array}{l}1.03 \\
(2.67)\end{array}$ & $\begin{array}{c}0.45 \\
(3.44)\end{array}$ \\
\hline 5(High) & 1.15 & 1.05 & 1.85 & 2.73 & 0.88 & $\begin{array}{c}1.71 \\
(3.18)\end{array}$ & $\begin{array}{c}1.09 \\
(5.81)\end{array}$ \\
\hline High-Low & -0.01 & 0.07 & 0.56 & 1.67 & 0.57 & $\begin{array}{c}1.03 \\
(3.09)\end{array}$ & $\begin{array}{c}0.94 \\
(3.05)\end{array}$ \\
\hline
\end{tabular}


Table 7: Average returns of predictive double-sorted portfolios

The table reports equal-weighted average returns for predictive double-sorted portfolios. The sample consists of the 985 individual stocks included in the S\&P 500 index over 1993-2010. For each month, all stocks in the sample are first sorted into five quintiles on the basis of one control variable. Within each quintile, the stocks are then sorted into five quintiles according to their betas. These five beta portfolios are then averaged across the five control variable portfolios to produce beta portfolios with large cross-portfolio variation in their betas but little variation in the control variable. $\beta^{s}, \beta^{c}, \beta^{d}$, and $\beta^{n}$ are the standard, continuous, discontinuous, and overnight betas, respectively. ME denotes the logarithm of the market capitalization of firms. $\mathrm{BM}$ denotes the ratio of the book value of common equity to the market value of equity. MOM is the compound gross return from month $t-11$ through month $t-1$. REV is the month $t$ return. IVOL is a measure of idiosyncratic volatility. CSK and CKT are the measures of coskewness and cokurtosis, respectively. RSK and RKT denote the realized skewness and realized kurtosis computed from high-frequency data. MAX represents the maximum daily raw return for month $t$. ILLIQ refers to the logarithm of the average daily ratio of the absolute stock return to the dollar trading volume from month $t-11$ through month $t$. The first five rows in each panel report time-series averages of monthly excess returns for the beta quintile portfolios. The row labeled "High-Low" reports the difference in the returns between portfolio 5 and portfolio 1. The row labeled "FFC4 alpha" reports the average Fama-French-Carhart four-factor alphas. The corresponding Newey-West robust $t$-statistics are reported in parentheses. Panels A, B, C, and D display the results for the portfolios first sorted by the control variables listed in the columns and then by $\beta^{s}, \beta^{c}, \beta^{d}$, and $\beta^{n}$, respectively.

\begin{tabular}{|c|c|c|c|c|c|c|c|c|c|c|c|}
\hline & $\mathrm{ME}$ & $\mathrm{BM}$ & MOM & REV & IVOL & CSK & CKT & RSK & RKT & MAX & ILLIQ \\
\hline \multicolumn{12}{|c|}{ Panel A: Final sort by $\beta^{s}$} \\
\hline 1 (Low) & 0.74 & 0.72 & 0.92 & 0.73 & 0.89 & 0.85 & 0.72 & 0.69 & 0.59 & 0.87 & 0.77 \\
\hline 2 & 0.93 & 0.88 & 0.96 & 0.97 & 1.02 & 0.82 & 0.81 & 0.81 & 0.86 & 0.93 & 0.86 \\
\hline 3 & 0.95 & 1.00 & 1.11 & 0.97 & 1.04 & 0.96 & 1.00 & 0.98 & 0.95 & 0.93 & 0.98 \\
\hline 4 & 1.20 & 1.18 & 1.13 & 1.27 & 1.19 & 1.20 & 1.14 & 1.32 & 1.23 & 1.22 & 1.17 \\
\hline 5 (High) & 1.61 & 1.58 & 1.24 & 1.52 & 1.30 & 1.61 & 1.75 & 1.50 & 1.67 & 1.48 & 1.63 \\
\hline High-Low & $\begin{array}{c}0.87 \\
(1.54)\end{array}$ & $\begin{array}{c}0.86 \\
(1.64)\end{array}$ & $\begin{array}{c}0.32 \\
(1.47)\end{array}$ & $\begin{array}{c}0.78 \\
(1.55)\end{array}$ & $\begin{array}{c}0.41 \\
(1.01)\end{array}$ & $\begin{array}{c}0.76 \\
(1.43)\end{array}$ & $\begin{array}{c}1.03 \\
(1.91)\end{array}$ & $\begin{array}{c}0.81 \\
(1.36)\end{array}$ & $\begin{array}{c}1.08 \\
(1.84)\end{array}$ & $\begin{array}{c}0.61 \\
(1.58)\end{array}$ & $\begin{array}{c}0.86 \\
(1.57)\end{array}$ \\
\hline FFC4 alpha & $\begin{array}{c}0.63 \\
(2.01)\end{array}$ & $\begin{array}{c}0.57 \\
(1.87)\end{array}$ & $\begin{array}{c}0.08 \\
(1.33)\end{array}$ & $\begin{array}{c}0.52 \\
(1.33)\end{array}$ & $\begin{array}{c}0.22 \\
(0.84)\end{array}$ & $\begin{array}{c}0.46 \\
(1.50)\end{array}$ & $\begin{array}{c}0.70 \\
(2.34)\end{array}$ & $\begin{array}{c}0.52 \\
(1.56)\end{array}$ & $\begin{array}{c}0.77 \\
(2.30)\end{array}$ & $\begin{array}{c}0.42 \\
(1.72)\end{array}$ & $\begin{array}{c}0.60 \\
(2.19)\end{array}$ \\
\hline \multicolumn{12}{|c|}{ Panel B: Final sort by $\beta^{c}$} \\
\hline 1 (Low) & 0.76 & 0.74 & 0.93 & 0.81 & 0.88 & 0.81 & 0.80 & 0.77 & 0.75 & 0.90 & 0.80 \\
\hline 2 & 0.90 & 0.86 & 0.96 & 0.93 & 0.96 & 0.88 & 0.81 & 0.87 & 0.87 & 0.87 & 0.78 \\
\hline 3 & 0.90 & 0.98 & 0.97 & 0.91 & 1.02 & 0.93 & 0.95 & 0.94 & 0.88 & 1.00 & 0.97 \\
\hline 4 & 1.10 & 1.14 & 1.19 & 1.10 & 1.11 & 1.06 & 1.06 & 1.19 & 1.19 & 1.04 & 1.06 \\
\hline 5 (High) & 1.57 & 1.45 & 1.23 & 1.56 & 1.30 & 1.59 & 1.65 & 1.49 & 1.60 & 1.46 & 1.64 \\
\hline High-Low & $\begin{array}{c}0.81 \\
(1.46)\end{array}$ & $\begin{array}{c}0.71 \\
(1.42)\end{array}$ & $\begin{array}{c}0.30 \\
(1.61)\end{array}$ & $\begin{array}{c}0.74 \\
(1.52)\end{array}$ & $\begin{array}{c}0.42 \\
(1.08)\end{array}$ & $\begin{array}{c}0.78 \\
(1.50)\end{array}$ & $\begin{array}{c}0.85 \\
(1.67)\end{array}$ & $\begin{array}{c}0.72 \\
(1.27)\end{array}$ & $\begin{array}{c}0.85 \\
(1.51)\end{array}$ & $\begin{array}{c}0.55 \\
(1.47)\end{array}$ & $\begin{array}{c}0.84 \\
(1.53)\end{array}$ \\
\hline FFC4 alpha & $\begin{array}{c}0.60 \\
(1.75)\end{array}$ & $\begin{array}{c}0.42 \\
(1.35)\end{array}$ & $\begin{array}{c}0.13 \\
(1.44)\end{array}$ & $\begin{array}{c}0.51 \\
(1.66)\end{array}$ & $\begin{array}{c}0.25 \\
(0.90)\end{array}$ & $\begin{array}{c}0.51 \\
(1.55)\end{array}$ & $\begin{array}{c}0.55 \\
(1.72)\end{array}$ & $\begin{array}{c}0.49 \\
(1.45)\end{array}$ & $\begin{array}{c}0.57 \\
(1.66)\end{array}$ & $\begin{array}{c}0.37 \\
(1.50)\end{array}$ & $\begin{array}{c}0.60 \\
(2.11)\end{array}$ \\
\hline \multicolumn{12}{|c|}{ Panel C: Final sort by $\beta^{d}$} \\
\hline 1 (Low) & 0.74 & 0.70 & 0.82 & 0.72 & 0.82 & 0.73 & 0.71 & 0.74 & 0.70 & 0.74 & 0.73 \\
\hline 2 & 0.88 & 0.76 & 0.90 & 0.81 & 0.79 & 0.81 & 0.85 & 0.78 & 0.78 & 0.74 & 0.87 \\
\hline 3 & 0.96 & 0.92 & 0.97 & 0.90 & 1.12 & 0.96 & 0.88 & 0.90 & 0.92 & 1.06 & 0.93 \\
\hline 4 & 1.19 & 1.17 & 1.15 & 1.12 & 1.17 & 1.07 & 1.11 & 1.18 & 1.12 & 1.26 & 1.13 \\
\hline 5 (High) & 1.47 & 1.62 & 1.30 & 1.73 & 1.33 & 1.68 & 1.70 & 1.66 & 1.75 & 1.46 & 1.56 \\
\hline High-Low & $\begin{array}{c}0.73 \\
(1.32)\end{array}$ & $\begin{array}{c}0.92 \\
(1.70)\end{array}$ & $\begin{array}{c}0.48 \\
(1.95)\end{array}$ & $\begin{array}{c}1.02 \\
(2.02)\end{array}$ & $\begin{array}{c}0.50 \\
(1.36)\end{array}$ & $\begin{array}{c}0.95 \\
(1.79)\end{array}$ & $\begin{array}{c}0.99 \\
(1.87)\end{array}$ & $\begin{array}{c}0.92 \\
(1.58)\end{array}$ & $\begin{array}{c}1.05 \\
(1.84)\end{array}$ & $\begin{array}{c}0.72 \\
(1.90)\end{array}$ & $\begin{array}{c}0.83 \\
(1.78)\end{array}$ \\
\hline FFC4 alpha & $\begin{array}{c}0.51 \\
(1.70)\end{array}$ & $\begin{array}{c}0.60 \\
(2.10)\end{array}$ & $\begin{array}{c}0.26 \\
(1.92)\end{array}$ & $\begin{array}{c}0.73 \\
(2.59)\end{array}$ & $\begin{array}{c}0.28 \\
(1.21)\end{array}$ & $\begin{array}{c}0.65 \\
(2.29)\end{array}$ & $\begin{array}{c}0.66 \\
(2.33)\end{array}$ & $\begin{array}{c}0.61 \\
(1.95)\end{array}$ & $\begin{array}{c}0.73 \\
(2.43)\end{array}$ & $\begin{array}{c}0.48 \\
(2.12)\end{array}$ & $\begin{array}{c}0.56 \\
(2.06)\end{array}$ \\
\hline \multicolumn{12}{|c|}{ Panel D: Final sort by $\beta^{n}$} \\
\hline 1 (Low) & 0.69 & 0.64 & 0.79 & 0.66 & 0.72 & 0.62 & 0.63 & 0.63 & 0.64 & 0.66 & 0.68 \\
\hline 2 & 0.78 & 0.74 & 0.86 & 0.75 & 0.93 & 0.83 & 0.82 & 0.81 & 0.79 & 0.86 & 0.82 \\
\hline 3 & 0.95 & 0.90 & 1.05 & 0.95 & 0.99 & 0.91 & 0.95 & 0.93 & 0.87 & 0.99 & 0.94 \\
\hline 4 & 1.18 & 1.13 & 1.12 & 1.13 & 1.20 & 1.18 & 1.15 & 1.12 & 1.12 & 1.20 & 1.05 \\
\hline 5 (High) & 1.63 & 1.75 & 1.44 & 1.78 & 1.40 & 1.70 & 1.70 & 1.75 & 1.85 & 1.53 & 1.75 \\
\hline High-Low & $\begin{array}{c}0.94 \\
(1.83)\end{array}$ & $\begin{array}{c}1.10 \\
(2.20)\end{array}$ & $\begin{array}{c}0.65 \\
(2.11)\end{array}$ & $\begin{array}{c}1.12 \\
(2.35)\end{array}$ & $\begin{array}{c}0.68 \\
(1.99)\end{array}$ & $\begin{array}{c}1.07 \\
(2.09)\end{array}$ & $\begin{array}{c}1.07 \\
(2.13)\end{array}$ & $\begin{array}{c}1.12 \\
(2.00)\end{array}$ & $\begin{array}{c}1.21 \\
(2.24)\end{array}$ & $\begin{array}{c}0.86 \\
(2.46)\end{array}$ & $\begin{array}{c}1.07 \\
(2.04)\end{array}$ \\
\hline FFC4 alpha & $\begin{array}{c}0.76 \\
(2.57)\end{array}$ & $\begin{array}{c}0.83 \\
(2.96)\end{array}$ & $\begin{array}{c}0.43 \\
(1.98)\end{array}$ & $\begin{array}{c}0.86 \\
(3.01)\end{array}$ & $\begin{array}{c}0.50 \\
(2.24)\end{array}$ & $\begin{array}{c}0.80 \\
(2.68)\end{array}$ & $\begin{array}{c}0.78 \\
(2.74)\end{array}$ & $\begin{array}{c}0.85 \\
(2.81)\end{array}$ & $\begin{array}{c}0.91 \\
(3.04)\end{array}$ & $\begin{array}{c}0.66 \\
(3.01)\end{array}$ & $\begin{array}{c}0.85 \\
(3.17)\end{array}$ \\
\hline
\end{tabular}


Table 8: Fama-MacBeth regressions

The table reports the estimated regression coefficients and robust $t$-statistics (in parentheses) from Fama-MacBeth crosssectional regressions for monthly stock returns. The sample consists of the 985 individual stocks included in the S\&P 500 index over 1993-2010. $\beta^{s}, \beta^{c}, \beta^{d}$, and $\beta^{n}$ are the standard, continuous, discontinuous, and overnight betas, respectively. ME denotes the logarithm of the market capitalization of firms. BM denotes the ratio of the book value of common equity to the market value of equity. MOM is the compound gross return from month $t-11$ through month $t-1$. REV is the month $t$ return. IVOL is a measure of idiosyncratic volatility. CSK and CKT denote the measures of coskewness and cokurtosis, respectively. RSK and RKT are the realized skewness and realized kurtosis, respectively, computed from high-frequency data. MAX represents the maximum daily raw return for month $t$. ILLIQ refers to the logarithm of the average daily ratio of the absolute stock return to the dollar trading volume from month $t-11$ through month $t$. Panel A reports the results of simple regressions with a single explanatory variable. Panel B reports the results of multiple regressions with more than one explanatory variable.

Panel A: Simple regressions

\begin{tabular}{|c|c|c|c|c|c|c|c|c|c|c|c|c|c|c|}
\hline$\beta^{s}$ & $\beta^{c}$ & $\beta^{d}$ & $\beta^{n}$ & $\mathrm{ME}$ & $\mathrm{BM}$ & MOM & REV & IVOL & CSK & CKT & RSK & RKT & MAX & ILLIQ \\
\hline $\begin{array}{c}0.86 \\
(1.94)\end{array}$ & $\begin{array}{c}0.95 \\
(1.91)\end{array}$ & $\begin{array}{c}0.82 \\
(1.99)\end{array}$ & $\begin{array}{c}0.58 \\
(2.14)\end{array}$ & $\begin{array}{c}-0.37 \\
(-4.08)\end{array}$ & $\begin{array}{l}-0.20 \\
(-0.79)\end{array}$ & $\begin{array}{c}0.01 \\
(0.82)\end{array}$ & $\begin{array}{c}-0.01 \\
(-0.74)\end{array}$ & $\begin{array}{c}0.23 \\
(1.36)\end{array}$ & $\begin{array}{l}-1.56 \\
(-1.83)\end{array}$ & $\begin{array}{l}-0.25 \\
(-1.43)\end{array}$ & $\begin{array}{c}-0.63 \\
(-1.91)\end{array}$ & $\begin{array}{c}0.15 \\
(1.94)\end{array}$ & $\begin{array}{c}0.06 \\
(1.28)\end{array}$ & $\begin{array}{c}0.31 \\
(3.33)\end{array}$ \\
\hline
\end{tabular}

Panel B: Multiple regressions

\begin{tabular}{|c|c|c|c|c|c|c|c|c|c|c|c|c|c|c|c|}
\hline Regression & $\beta^{s}$ & $\beta^{c}$ & $\beta^{d}$ & $\beta^{n}$ & $\mathrm{ME}$ & $\mathrm{BM}$ & MOM & REV & IVOL & CSK & CKT & RSK & $\mathrm{RKT}$ & MAX & ILLIQ \\
\hline I & $\begin{array}{c}0.71 \\
(1.41)\end{array}$ & & & & $\begin{array}{c}-0.45 \\
(-3.03)\end{array}$ & $\begin{array}{c}-0.28 \\
(-1.51)\end{array}$ & $\begin{array}{c}0.01 \\
(1.31)\end{array}$ & $\begin{array}{c}-0.02 \\
(-2.06)\end{array}$ & $\begin{array}{c}-0.19 \\
(-1.73)\end{array}$ & $\begin{array}{c}0.77 \\
(1.35)\end{array}$ & $\begin{array}{c}-0.41 \\
(-2.25)\end{array}$ & $\begin{array}{c}-0.18 \\
(-0.67)\end{array}$ & $\begin{array}{c}-0.05 \\
(-0.92)\end{array}$ & $\begin{array}{c}0.02 \\
(0.69)\end{array}$ & $\begin{array}{c}-0.05 \\
(-0.43)\end{array}$ \\
\hline II & & $\begin{array}{c}0.58 \\
(1.46)\end{array}$ & & & $\begin{array}{c}-0.47 \\
(-3.03)\end{array}$ & $\begin{array}{c}-0.31 \\
(-1.66)\end{array}$ & $\begin{array}{c}0.01 \\
(1.19)\end{array}$ & $\begin{array}{c}-0.02 \\
(-2.04)\end{array}$ & $\begin{array}{c}-0.15 \\
(-1.36)\end{array}$ & $\begin{array}{c}0.78 \\
(1.39)\end{array}$ & $\begin{array}{c}-0.18 \\
(-1.27)\end{array}$ & $\begin{array}{c}-0.21 \\
(-0.78)\end{array}$ & $\begin{array}{l}-0.05 \\
(-1.00)\end{array}$ & $\begin{array}{c}0.03 \\
(0.93)\end{array}$ & $\begin{array}{c}-0.03 \\
(-0.22)\end{array}$ \\
\hline III & & & $\begin{array}{c}0.55 \\
(1.97)\end{array}$ & & $\begin{array}{c}-0.49 \\
(-3.10)\end{array}$ & $\begin{array}{c}-0.33 \\
(-1.71)\end{array}$ & $\begin{array}{c}0.01 \\
(1.27)\end{array}$ & $\begin{array}{l}-0.02 \\
(-2.07)\end{array}$ & $\begin{array}{c}-0.17 \\
(-1.62)\end{array}$ & $\begin{array}{c}0.97 \\
(1.67)\end{array}$ & $\begin{array}{l}-0.15 \\
(-1.06)\end{array}$ & $\begin{array}{l}-0.18 \\
(-0.66)\end{array}$ & $\begin{array}{l}-0.04 \\
(-0.77)\end{array}$ & $\begin{array}{c}0.03 \\
(0.80)\end{array}$ & $\begin{array}{c}-0.10 \\
(-0.73)\end{array}$ \\
\hline IV & & & & $\begin{array}{c}0.43 \\
(2.25)\end{array}$ & $\begin{array}{c}-0.51 \\
(-3.09)\end{array}$ & $\begin{array}{c}-0.32 \\
(-1.63)\end{array}$ & $\begin{array}{c}0.01 \\
(1.45)\end{array}$ & $\begin{array}{c}-0.03 \\
(-2.16)\end{array}$ & $\begin{array}{c}-0.17 \\
(-1.56)\end{array}$ & $\begin{array}{c}0.86 \\
(1.48)\end{array}$ & $\begin{array}{c}-0.13 \\
(-0.90)\end{array}$ & $\begin{array}{l}-0.12 \\
(-0.47)\end{array}$ & $\begin{array}{c}-0.03 \\
(-0.62)\end{array}$ & $\begin{array}{c}0.02 \\
(0.50)\end{array}$ & $\begin{array}{c}-0.11 \\
(-0.81)\end{array}$ \\
\hline $\mathrm{V}$ & & $\begin{array}{c}-0.06 \\
(-0.13)\end{array}$ & $\begin{array}{c}0.29 \\
(1.68)\end{array}$ & $\begin{array}{c}0.30 \\
(2.16)\end{array}$ & $\begin{array}{c}-0.37 \\
(-4.45)\end{array}$ & $\begin{array}{c}-0.25 \\
(-0.97)\end{array}$ & $\begin{array}{c}0.01 \\
(1.15)\end{array}$ & & & & & & & & \\
\hline $\mathrm{V}$ & & $\begin{array}{c}-0.02 \\
(-0.04)\end{array}$ & $\begin{array}{c}0.28 \\
(1.74)\end{array}$ & $\begin{array}{c}0.31 \\
(2.22)\end{array}$ & $\begin{array}{c}-0.38 \\
(-4.59)\end{array}$ & $\begin{array}{c}-0.23 \\
(-1.19)\end{array}$ & $\begin{array}{c}0.00 \\
(0.97)\end{array}$ & $\begin{array}{c}-0.02 \\
(-2.17)\end{array}$ & & & & & & & \\
\hline VII & & $\begin{array}{l}-0.05 \\
(-0.12)\end{array}$ & $\begin{array}{c}0.36 \\
(1.61)\end{array}$ & $\begin{array}{c}0.32 \\
(2.11)\end{array}$ & $\begin{array}{c}-0.38 \\
(-4.96)\end{array}$ & $\begin{array}{c}-0.26 \\
(-1.03)\end{array}$ & $\begin{array}{c}0.01 \\
(1.16)\end{array}$ & & $\begin{array}{c}-0.10 \\
(-0.99)\end{array}$ & & & & & & \\
\hline VIII & & $\begin{array}{l}-0.07 \\
(-0.15)\end{array}$ & $\begin{array}{c}0.33 \\
(1.72)\end{array}$ & $\begin{array}{c}0.29 \\
(2.01)\end{array}$ & $\begin{array}{c}-0.37 \\
(-4.50)\end{array}$ & $\begin{array}{c}-0.28 \\
(-1.12)\end{array}$ & $\begin{array}{c}0.01 \\
(1.15)\end{array}$ & & & $\begin{array}{c}-0.14 \\
(-0.26)\end{array}$ & & & & & \\
\hline IX & & $\begin{array}{l}-0.01 \\
(-0.03)\end{array}$ & $\begin{array}{c}0.30 \\
(1.76)\end{array}$ & $\begin{array}{c}0.28 \\
(2.02)\end{array}$ & $\begin{array}{c}-0.36 \\
(-4.70)\end{array}$ & $\begin{array}{c}-0.28 \\
(-1.14)\end{array}$ & $\begin{array}{c}0.01 \\
(1.12)\end{array}$ & & & & $\begin{array}{c}-0.05 \\
(-0.36)\end{array}$ & & & & \\
\hline $\mathrm{X}$ & & $\begin{array}{c}-0.02 \\
(-0.04)\end{array}$ & $\begin{array}{c}0.28 \\
(1.71)\end{array}$ & $\begin{array}{c}0.30 \\
(2.05)\end{array}$ & $\begin{array}{c}-0.37 \\
(-4.49)\end{array}$ & $\begin{array}{c}-0.27 \\
(-1.04)\end{array}$ & $\begin{array}{c}0.01 \\
(1.11)\end{array}$ & & & & & $\begin{array}{c}-0.57 \\
(-1.94)\end{array}$ & & & \\
\hline XI & & $\begin{array}{c}-0.07 \\
(-0.15)\end{array}$ & $\begin{array}{c}0.27 \\
(1.73)\end{array}$ & $\begin{array}{c}0.29 \\
(2.00)\end{array}$ & $\begin{array}{c}-0.40 \\
(-4.69)\end{array}$ & $\begin{array}{c}-0.31 \\
(-1.28)\end{array}$ & $\begin{array}{c}0.01 \\
(1.27)\end{array}$ & & & & & & $\begin{array}{c}-0.05 \\
(-0.85)\end{array}$ & & \\
\hline XII & & $\begin{array}{l}-0.03 \\
(-0.07)\end{array}$ & $\begin{array}{c}0.39 \\
(1.74)\end{array}$ & $\begin{array}{c}0.35 \\
(2.47)\end{array}$ & $\begin{array}{c}-0.38 \\
(-4.86)\end{array}$ & $\begin{array}{c}-0.26 \\
(-1.02)\end{array}$ & $\begin{array}{c}0.01 \\
(1.14)\end{array}$ & & & & & & & $\begin{array}{c}-0.05 \\
(-1.68)\end{array}$ & \\
\hline XIII & & $\begin{array}{l}-0.06 \\
(-0.13)\end{array}$ & $\begin{array}{c}0.29 \\
(1.76)\end{array}$ & $\begin{array}{c}0.28 \\
(1.96)\end{array}$ & $\begin{array}{c}-0.42 \\
(-2.65)\end{array}$ & $\begin{array}{c}-0.24 \\
(-0.92)\end{array}$ & $\begin{array}{c}0.01 \\
(1.34)\end{array}$ & & & & & & & & $\begin{array}{c}-0.06 \\
(-0.48)\end{array}$ \\
\hline XIV & & $\begin{array}{l}0.02 \\
0.05\end{array}$ & $\begin{array}{r}0.31 \\
\quad(2 .\end{array}$ & $\begin{array}{c}0.31 \\
33)\end{array}$ & $\begin{array}{c}-0.47 \\
(-3.17)\end{array}$ & $\begin{array}{c}-0.28 \\
(-1.48)\end{array}$ & $\begin{array}{c}0.01 \\
(1.25)\end{array}$ & $\begin{array}{c}-0.02 \\
(-2.09)\end{array}$ & $\begin{array}{c}-0.20 \\
(-1.93)\end{array}$ & $\begin{array}{c}0.80 \\
(1.44)\end{array}$ & $\begin{array}{c}-0.13 \\
(-0.91)\end{array}$ & $\begin{array}{c}-0.18 \\
(-0.69)\end{array}$ & $\begin{array}{c}-0.04 \\
(-0.80)\end{array}$ & $\begin{array}{c}0.03 \\
(0.77)\end{array}$ & $\begin{array}{c}-0.08 \\
(-0.61)\end{array}$ \\
\hline $\mathrm{XV}$ & & 0.25 & $\begin{array}{r}0.25 \\
(1.96)\end{array}$ & 0.25 & $\begin{array}{c}-0.45 \\
(-2.95)\end{array}$ & $\begin{array}{c}-0.28 \\
(-1.46)\end{array}$ & $\begin{array}{c}0.56 \\
(1.33)\end{array}$ & $\begin{array}{c}-0.02 \\
(-2.09)\end{array}$ & $\begin{array}{c}-0.21 \\
(-2.00)\end{array}$ & $\begin{array}{c}0.81 \\
(1.41)\end{array}$ & $\begin{array}{c}-0.17 \\
(-1.26)\end{array}$ & $\begin{array}{c}-0.18 \\
(-0.66)\end{array}$ & $\begin{array}{c}-0.02 \\
(-0.39)\end{array}$ & $\begin{array}{c}0.02 \\
(0.49)\end{array}$ & $\begin{array}{c}-0.06 \\
(-0.48)\end{array}$ \\
\hline
\end{tabular}


Table 9: Fama-MacBeth regressions with different beta estimation frequencies

The table reports the estimated regression coefficients and robust $t$-statistics (in parentheses) from monthly Fama-MacBeth cross-sectional regressions simultaneously controlling for all explanatory variables, restricting the coefficients for $\beta^{d}$ and $\beta^{n}$ to be the same. The sample consists of the 985 individual stocks included in the S\&P 500 index over 1993-2010. $\beta^{c}, \beta^{d}$, and $\beta^{n}$ refer to the continuous, discontinuous, and overnight betas, respectively. ME denotes the logarithm of the market capitalization of firms. BM denotes the ratio of the book value of common equity to the market value of equity. MOM is the compound gross return from month $t-11$ through month $t-1$. REV is the month $t$ return. IVOL is a measure of idiosyncratic volatility. CSK and CKT denote the measures of coskewness and cokurtosis, respectively. RSK and RKT refer to the realized skewness and realized kurtosis, respectively, computed from high-frequency data. MAX represents the maximum daily raw return for month $t$. ILLIQ refers to the logarithm of the average daily ratio of the absolute stock return to the dollar trading volume from month $t-11$ through month $t$. Panel A reports the results for different $\beta^{c}$ estimates computed using the sampling frequencies listed in the first column labeled "Frequency." Panel B reports the results for different $\beta^{d}$ estimates based on the sampling frequencies in the "Frequency" column.

Panel A: Different $\beta^{c}$ estimates

\begin{tabular}{|c|c|c|c|c|c|c|c|c|c|c|c|c|c|}
\hline Frequency & $\beta^{c}$ & $\beta^{d}, \beta^{n}$ & $\mathrm{ME}$ & $\mathrm{BM}$ & $\mathrm{MOM}$ & REV & IVOL & CSK & CKT & RSK & RKT & MAX & ILLIQ \\
\hline $5 \mathrm{mi}$ & $\begin{array}{l}-0.36 \\
(-0.87)\end{array}$ & $\begin{array}{c}0.34 \\
(2.57)\end{array}$ & $\begin{array}{l}-0.52 \\
(-3.34)\end{array}$ & $\begin{array}{l}-0.30 \\
(-1.60)\end{array}$ & $\begin{array}{c}0.01 \\
(1.37)\end{array}$ & $\begin{array}{l}-2.45 \\
(-2.13)\end{array}$ & $\begin{array}{c}-0.21 \\
(-1.96)\end{array}$ & $\begin{array}{c}0.86 \\
(1.54)\end{array}$ & $\begin{array}{l}-0.06 \\
(-0.42)\end{array}$ & $\begin{array}{c}-0.15 \\
(-0.55)\end{array}$ & $\begin{array}{l}-0.04 \\
(-0.73)\end{array}$ & $\begin{array}{c}0.02 \\
(0.69)\end{array}$ & $\begin{array}{l}-0.16 \\
(-1.21)\end{array}$ \\
\hline $25 \min$ & $\begin{array}{l}-0.02 \\
(-0.04)\end{array}$ & $\begin{array}{c}0.31 \\
(2.35)\end{array}$ & $\begin{array}{c}-0.51 \\
(-3.37)\end{array}$ & $\begin{array}{c}-0.31 \\
(-1.66)\end{array}$ & $\begin{array}{c}0.01 \\
(1.36)\end{array}$ & $\begin{array}{l}-2.39 \\
(-2.10)\end{array}$ & $\begin{array}{c}-0.21 \\
(-2.01)\end{array}$ & $\begin{array}{c}0.82 \\
(1.50)\end{array}$ & $\begin{array}{l}-0.08 \\
(-0.61)\end{array}$ & $\begin{array}{c}-0.21 \\
(-0.79)\end{array}$ & $\begin{array}{l}-0.03 \\
(-0.69)\end{array}$ & $\begin{array}{c}0.03 \\
(0.73)\end{array}$ & $\begin{array}{l}-0.10 \\
(-0.82)\end{array}$ \\
\hline $75 \min$ & $\begin{array}{c}0.02 \\
(0.05)\end{array}$ & $\begin{array}{c}0.31 \\
(2.33)\end{array}$ & $\begin{array}{c}-0.47 \\
(-3.17)\end{array}$ & $\begin{array}{c}-0.28 \\
(-1.48)\end{array}$ & $\begin{array}{c}0.01 \\
(1.25)\end{array}$ & $\begin{array}{l}-2.38 \\
(-2.09)\end{array}$ & $\begin{array}{c}-0.20 \\
(-1.94)\end{array}$ & $\begin{array}{c}0.80 \\
(1.44)\end{array}$ & $\begin{array}{c}-0.13 \\
(-0.91)\end{array}$ & $\begin{array}{c}-0.18 \\
(-0.69)\end{array}$ & $\begin{array}{c}-0.04 \\
(-0.80)\end{array}$ & $\begin{array}{c}0.03 \\
(0.77)\end{array}$ & $\begin{array}{l}-0.08 \\
(-0.61)\end{array}$ \\
\hline $125 \mathrm{~min}$ & $\begin{array}{c}0.12 \\
(0.35)\end{array}$ & $\begin{array}{c}0.27 \\
(2.10)\end{array}$ & $\begin{array}{l}-0.47 \\
(-3.10)\end{array}$ & $\begin{array}{l}-0.30 \\
(-1.58)\end{array}$ & $\begin{array}{c}0.01 \\
(1.34)\end{array}$ & $\begin{array}{l}-2.43 \\
(-2.11)\end{array}$ & $\begin{array}{l}-0.21 \\
(-1.99)\end{array}$ & $\begin{array}{c}0.82 \\
(1.46)\end{array}$ & $\begin{array}{l}-0.15 \\
(-1.05)\end{array}$ & $\begin{array}{c}-0.17 \\
(-0.65)\end{array}$ & $\begin{array}{l}-0.03 \\
(-0.59)\end{array}$ & $\begin{array}{c}0.02 \\
(0.62)\end{array}$ & $\begin{array}{l}-0.08 \\
(-0.60)\end{array}$ \\
\hline $180 \mathrm{~min}$ & $\begin{array}{c}0.05 \\
(0.14)\end{array}$ & $\begin{array}{c}0.29 \\
(2.22)\end{array}$ & $\begin{array}{l}-0.48 \\
(-3.17)\end{array}$ & $\begin{array}{l}-0.31 \\
(-1.62)\end{array}$ & $\begin{array}{c}0.01 \\
(1.40)\end{array}$ & $\begin{array}{l}-2.52 \\
(-2.20)\end{array}$ & $\begin{array}{l}-0.20 \\
(-1.94)\end{array}$ & $\begin{array}{c}0.88 \\
(1.59)\end{array}$ & $\begin{array}{l}-0.15 \\
(-1.11)\end{array}$ & $\begin{array}{c}-0.17 \\
(-0.62)\end{array}$ & $\begin{array}{l}-0.03 \\
(-0.64)\end{array}$ & $\begin{array}{c}0.02 \\
(0.68)\end{array}$ & $\begin{array}{c}-0.10 \\
(-0.73)\end{array}$ \\
\hline $\operatorname{mix}$ & $\begin{array}{c}0.06 \\
(0.17)\end{array}$ & $\begin{array}{c}0.30 \\
(2.24)\end{array}$ & $\begin{array}{l}-0.42 \\
(-2.87)\end{array}$ & $\begin{array}{c}-0.31 \\
(-1.63)\end{array}$ & $\begin{array}{c}0.01 \\
(1.42)\end{array}$ & $\begin{array}{l}-2.49 \\
(-2.18)\end{array}$ & $\begin{array}{c}-0.21 \\
(-1.96)\end{array}$ & $\begin{array}{c}0.86 \\
(1.53)\end{array}$ & $\begin{array}{c}-0.09 \\
(-0.66)\end{array}$ & $\begin{array}{c}-0.18 \\
(-0.67)\end{array}$ & $\begin{array}{c}-0.03 \\
(-0.63)\end{array}$ & $\begin{array}{c}0.02 \\
(0.61)\end{array}$ & $\begin{array}{c}-0.08 \\
(-0.58)\end{array}$ \\
\hline
\end{tabular}

Panel B: Different $\beta^{d}$ estimates

\begin{tabular}{|c|c|c|c|c|c|c|c|c|c|c|c|c|c|}
\hline Frequency & $\beta^{c}$ & $\beta^{d}, \beta^{n}$ & $\mathrm{ME}$ & $\mathrm{BM}$ & MOM & $\mathrm{REV}$ & IVOL & CSK & CKT & RSK & RKT & MAX & ILLIQ \\
\hline $5 \mathrm{~min}$ & $\begin{array}{c}0.12 \\
(0.32)\end{array}$ & $\begin{array}{c}0.28 \\
(2.10)\end{array}$ & $\begin{array}{l}-0.48 \\
(-3.19)\end{array}$ & $\begin{array}{l}-0.30 \\
(-1.57)\end{array}$ & $\begin{array}{c}0.01 \\
(1.28)\end{array}$ & $\begin{array}{l}-2.39 \\
(-2.10)\end{array}$ & $\begin{array}{l}-0.19 \\
(-1.80)\end{array}$ & $\begin{array}{c}0.78 \\
(1.40)\end{array}$ & $\begin{array}{c}-0.11 \\
(-0.81)\end{array}$ & $\begin{array}{l}-0.17 \\
(-0.66)\end{array}$ & $\begin{array}{l}-0.04 \\
(-0.70)\end{array}$ & $\begin{array}{c}0.03 \\
(0.76)\end{array}$ & $\begin{array}{l}-0.08 \\
(-0.61)\end{array}$ \\
\hline $25 \mathrm{~min}$ & $\begin{array}{c}0.05 \\
(0.15)\end{array}$ & $\begin{array}{c}0.29 \\
(2.32)\end{array}$ & $\begin{array}{l}-0.48 \\
(-3.19)\end{array}$ & $\begin{array}{l}-0.28 \\
(-1.47)\end{array}$ & $\begin{array}{c}0.01 \\
(1.30)\end{array}$ & $\begin{array}{l}-2.45 \\
(-2.14)\end{array}$ & $\begin{array}{l}-0.20 \\
(-1.88)\end{array}$ & $\begin{array}{c}0.80 \\
(1.44)\end{array}$ & $\begin{array}{c}-0.12 \\
(-0.87)\end{array}$ & $\begin{array}{c}-0.16 \\
(-0.59)\end{array}$ & $\begin{array}{c}-0.04 \\
(-0.76)\end{array}$ & $\begin{array}{c}0.03 \\
(0.80)\end{array}$ & $\begin{array}{l}-0.08 \\
(-0.61)\end{array}$ \\
\hline $75 \mathrm{~min}$ & $\begin{array}{c}0.02 \\
(0.05)\end{array}$ & $\begin{array}{c}0.31 \\
(2.33)\end{array}$ & $\begin{array}{l}-0.47 \\
(-3.17)\end{array}$ & $\begin{array}{l}-0.28 \\
(-1.48)\end{array}$ & $\begin{array}{c}0.01 \\
(1.25)\end{array}$ & $\begin{array}{l}-2.38 \\
(-2.09)\end{array}$ & $\begin{array}{l}-0.20 \\
(-1.94)\end{array}$ & $\begin{array}{c}0.80 \\
(1.44)\end{array}$ & $\begin{array}{l}-0.13 \\
(-0.91)\end{array}$ & $\begin{array}{l}-0.18 \\
(-0.69)\end{array}$ & $\begin{array}{l}-0.04 \\
(-0.80)\end{array}$ & $\begin{array}{c}0.03 \\
(0.77)\end{array}$ & $\begin{array}{l}-0.08 \\
(-0.61)\end{array}$ \\
\hline 125 & $\begin{array}{c}0.04 \\
(0.10)\end{array}$ & $\begin{array}{c}0.30 \\
(2.28)\end{array}$ & $\begin{array}{l}-0.47 \\
(-3.14)\end{array}$ & $\begin{array}{l}-0.28 \\
(-1.51)\end{array}$ & $\begin{array}{c}0.01 \\
(1.24)\end{array}$ & $\begin{array}{l}-2.44 \\
(-2.15)\end{array}$ & $\begin{array}{l}-0.21 \\
(-2.02)\end{array}$ & $\begin{array}{c}0.79 \\
(1.41)\end{array}$ & $\begin{array}{l}-0.14 \\
(-0.99)\end{array}$ & $\begin{array}{l}-0.19 \\
(-0.71)\end{array}$ & $\begin{array}{l}-0.04 \\
(-0.77)\end{array}$ & $\begin{array}{c}0.03 \\
(0.85)\end{array}$ & $\begin{array}{l}-0.07 \\
(-0.58)\end{array}$ \\
\hline $180 \mathrm{~min}$ & $\begin{array}{c}0.04 \\
(0.12)\end{array}$ & $\begin{array}{c}0.29 \\
(2.22)\end{array}$ & $\begin{array}{l}-0.47 \\
(-3.15)\end{array}$ & $\begin{array}{l}-0.28 \\
(-1.49)\end{array}$ & $\begin{array}{c}0.01 \\
(1.25)\end{array}$ & $\begin{array}{l}-2.47 \\
(-2.16)\end{array}$ & $\begin{array}{l}-0.21 \\
(-2.01)\end{array}$ & $\begin{array}{c}0.75 \\
(1.35)\end{array}$ & $\begin{array}{c}-0.13 \\
(-0.94)\end{array}$ & $\begin{array}{l}-0.20 \\
(-0.75)\end{array}$ & $\begin{array}{l}-0.04 \\
(-0.80)\end{array}$ & $\begin{array}{c}0.03 \\
(0.88)\end{array}$ & $\begin{array}{l}-0.07 \\
(-0.59)\end{array}$ \\
\hline $\operatorname{mix}$ & $\begin{array}{c}0.08 \\
(0.22)\end{array}$ & $\begin{array}{c}0.28 \\
(2.20)\end{array}$ & $\begin{array}{l}-0.45 \\
(-3.07)\end{array}$ & $\begin{array}{l}-0.28 \\
(-1.50)\end{array}$ & $\begin{array}{c}0.01 \\
(1.26)\end{array}$ & $\begin{array}{l}-2.44 \\
(-2.13)\end{array}$ & $\begin{array}{l}-0.20 \\
(-1.95)\end{array}$ & $\begin{array}{c}0.77 \\
(1.37)\end{array}$ & $\begin{array}{c}-0.13 \\
(-0.93)\end{array}$ & $\begin{array}{l}-0.18 \\
(-0.70)\end{array}$ & $\begin{array}{l}-0.04 \\
(-0.72)\end{array}$ & $\begin{array}{c}0.03 \\
(0.85)\end{array}$ & $\begin{array}{l}-0.07 \\
(-0.56)\end{array}$ \\
\hline
\end{tabular}


Table 10: Fama-MacBeth regressions with different beta estimation periods and return holding horizons

The table reports the estimated regression coefficients and robust $t$-statistics (in parentheses) from Fama-MacBeth crosssectional regressions for predicting the next $H$-month cumulative returns. The sample consists of the 985 individual stocks included in the S\&P 500 index over 1993-2010. The regressions simultaneously control for all explanatory variables, restricting the coefficients for $\beta^{d}$ and $\beta^{n}$ to be the same. The betas are computed from the previous $L$-month high-frequency returns. $\beta^{s}$, $\beta^{c}, \beta^{d}$, and $\beta^{n}$ refer to the standard, continuous, discontinuous, and overnight betas, respectively. ME denotes the logarithm of the market capitalization of firms. BM denotes the ratio of the book value of common equity to the market value of equity. MOM is the compound gross return from month $t-11$ through month $t-1$. REV is the month $t$ return. IVOL is a measure of idiosyncratic volatility. CSK and CKT denote the measures of coskewness and cokurtosis, respectively. RSK and RKT refer to the realized skewness and realized kurtosis, respectively, computed from high-frequency data. MAX represents the maximum daily raw return for month $t$. ILLIQ refers to the logarithm of the average daily ratio of the absolute stock return to the dollar trading volume from month $t-11$ through month $t$.

\begin{tabular}{|c|c|c|c|c|c|c|c|c|c|c|c|c|c|c|c|c|}
\hline Regr & $L$ & $H$ & $\beta^{s}$ & $\beta^{c}$ & $\beta^{n}$ & $\mathrm{ME}$ & $\mathrm{BM}$ & MOM & $\mathrm{iEV}$ & VOL & CSK & CKT & RSK & RKT & MAX & ILLIQ \\
\hline I & 3 & 1 & & & & & & & & & & & & & & \\
\hline II & 6 & 1 & $\begin{array}{r}0.6 \\
(1.6\end{array}$ & & & & & & & & & & & & $\begin{array}{c}0.06 \\
(1.55)\end{array}$ & $\begin{array}{c}0.10 \\
(0.70)\end{array}$ \\
\hline III & 12 & 3 & $\begin{array}{r}2.0 \\
(1.6\end{array}$ & & & & & $\begin{array}{c}0.02 \\
(1.37)\end{array}$ & & & & & & & & $\begin{array}{c}0.66 \\
(1.38)\end{array}$ \\
\hline IV & 12 & 6 & $\begin{array}{r}4.2 \\
(1.4\end{array}$ & & & & & $\begin{array}{c}0.03 \\
(1.09)\end{array}$ & & & & & & & & $\begin{array}{c}1.21 \\
(1.05)\end{array}$ \\
\hline V & 12 & 12 & $\begin{array}{c}9.57 \\
(1.42)\end{array}$ & & & $\begin{array}{l}-5 \\
(-1\end{array}$ & $\begin{array}{l}-3.76 \\
(-1.73)\end{array}$ & $\begin{array}{c}0.05 \\
(1.02)\end{array}$ & $\begin{array}{c}0.09 \\
(0.96)\end{array}$ & & $\begin{array}{c}3.73 \\
(0.79)\end{array}$ & $\begin{array}{l}-3.34 \\
(-1.77)\end{array}$ & & $\begin{array}{l}-0 \\
-0\end{array}$ & $\begin{array}{c}0.07 \\
(0.36)\end{array}$ & $\begin{array}{c}0.24 \\
(0.10)\end{array}$ \\
\hline $\mathrm{Vl}$ & 3 & 1 & & & 0 & $\begin{array}{l}-0 \\
-2\end{array}$ & $\begin{array}{l}-0.34 \\
(-1.69)\end{array}$ & $\begin{array}{c}0.00 \\
(0.94)\end{array}$ & $\begin{array}{c}-0.03 \\
(-2.78)\end{array}$ & $\begin{array}{l}-0.19 \\
(-1.68)\end{array}$ & $\begin{array}{c}0.76 \\
(1.24)\end{array}$ & $\begin{array}{l}-0.23 \\
(-1.66)\end{array}$ & $\begin{array}{l}-0.14 \\
(-0.48)\end{array}$ & $\begin{array}{l}-0.14 \\
(-2.53)\end{array}$ & $\begin{array}{c}0.05 \\
(1.49)\end{array}$ & $\begin{array}{c}0.07 \\
(0.53)\end{array}$ \\
\hline VII & 6 & 1 & & & $\begin{array}{c}0.55 \\
(4.15)\end{array}$ & $\begin{array}{c}-0.42 \\
(-2.63)\end{array}$ & $\begin{array}{c}-0.34 \\
(-1.70)\end{array}$ & $\begin{array}{c}0.00 \\
(0.90)\end{array}$ & $\begin{array}{c}-0.03 \\
(-2.87)\end{array}$ & $\begin{array}{c}-0.21 \\
(-1.91)\end{array}$ & $\begin{array}{c}0.85 \\
(1.37)\end{array}$ & $\begin{array}{c}-0.22 \\
(-1.61)\end{array}$ & $\begin{array}{c}-0.16 \\
(-0.56)\end{array}$ & $\begin{array}{c}-0.12 \\
(-2.26)\end{array}$ & $\begin{array}{c}0.06 \\
(1.70)\end{array}$ & $\begin{array}{c}0.05 \\
(0.39)\end{array}$ \\
\hline V & 12 & 3 & & $\begin{array}{l}-1.66 \\
(-1.39)\end{array}$ & $\begin{array}{c}1.65 \\
(3.94)\end{array}$ & $\begin{array}{c}-0.84 \\
(-1.86)\end{array}$ & $\begin{array}{c}-0.72 \\
(-1.60)\end{array}$ & $\begin{array}{c}0.01 \\
(1.27)\end{array}$ & $\begin{array}{c}-0.05 \\
(-1.97)\end{array}$ & $\begin{array}{c}-0.32 \\
(-1.52)\end{array}$ & $\begin{array}{c}2.50 \\
(1.86)\end{array}$ & $\begin{array}{c}-0.65 \\
(-2.12)\end{array}$ & $\begin{array}{c}-0.39 \\
(-0.74)\end{array}$ & $\begin{array}{c}-0.34 \\
(-2.85)\end{array}$ & $\begin{array}{c}0.14 \\
(1.92)\end{array}$ & $\begin{array}{c}0.46 \\
(1.11)\end{array}$ \\
\hline $\mathrm{I} 2$ & 1 & 6 & & $\begin{array}{l}-1.35 \\
(-0.50)\end{array}$ & $\begin{array}{c}2.73 \\
(3.45)\end{array}$ & $\begin{array}{l}-1.73 \\
(-1.67)\end{array}$ & $\begin{array}{l}-1.40 \\
(-1.59)\end{array}$ & $\begin{array}{c}0.03 \\
(1.06)\end{array}$ & $\begin{array}{c}-0.01 \\
(-0.23)\end{array}$ & $\begin{array}{c}0.02 \\
(0.06)\end{array}$ & $\begin{array}{c}4.01 \\
(1.64)\end{array}$ & $\begin{array}{l}-1.05 \\
(-1.65)\end{array}$ & $\begin{array}{c}-1.02 \\
(-1.00)\end{array}$ & $\begin{array}{c}-0.39 \\
(-1.40)\end{array}$ & $\begin{array}{c}0.05 \\
(0.50)\end{array}$ & $\begin{array}{c}0.96 \\
(0.99)\end{array}$ \\
\hline $\mathrm{X}$ & 12 & 12 & & $\begin{array}{c}3.82 \\
(0.65)\end{array}$ & $\begin{array}{c}4.07 \\
(3.28)\end{array}$ & $\begin{array}{l}-5.42 \\
(-2.04)\end{array}$ & $\begin{array}{c}-3.15 \\
(-1.59)\end{array}$ & $\begin{array}{c}0.05 \\
(1.00)\end{array}$ & $\begin{array}{c}0.08 \\
(0.89)\end{array}$ & $\begin{array}{c}-0.08 \\
(-0.11)\end{array}$ & $\begin{array}{c}4.85 \\
(1.01)\end{array}$ & $\begin{array}{l}-1.65 \\
(-1.09)\end{array}$ & $\begin{array}{l}-1.33 \\
(-0.72)\end{array}$ & $\begin{array}{l}-0.18 \\
(-0.23)\end{array}$ & $\begin{array}{c}0.08 \\
(0.39)\end{array}$ & $\begin{array}{c}0.35 \\
(0.17)\end{array}$ \\
\hline
\end{tabular}


Table 11: Fama-MacBeth regressions excluding macroeconomic news announcement days The table reports the estimated regression coefficients and robust $t$-statistics (in parentheses) from monthly Fama-MacBeth cross-sectional regressions simultaneously controlling for all explanatory variables, restricting the coefficients for $\beta^{d}$ and $\beta^{n}$ to be the same. The sample consists of the 985 individual stocks included in the S\&P500 index over 1993-2010. The betas are calculated excluding FOMC, Employment, and PPI announcement days in the estimation. $\beta^{c}, \beta^{d}$, and $\beta^{n}$ refer to the continuous, discontinuous, and overnight betas, respectively. ME denotes the logarithm of the market capitalization of firms. $\mathrm{BM}$ denotes the ratio of the book value of common equity to the market value of equity. MOM is the compound gross return from month $t-11$ through month $t-1$. REV is the month $t$ return. IVOL is a measure of idiosyncratic volatility. CSK and CKT denote the measures of coskewness and cokurtosis, respectively. RSK and RKT refer to the realized skewness and realized kurtosis, respectively, computed from high-frequency data. MAX represents the maximum daily raw return for month $t$. ILLIQ refers to the logarithm of the average daily ratio of the absolute stock return to the dollar trading volume from month $t-11$ through month $t$.

\begin{tabular}{|c|c|c|c|c|c|c|c|c|c|c|c|c|c|}
\hline Regression & $\beta^{c}$ & $\beta^{d}, \beta^{n}$ & $\mathrm{ME}$ & $\mathrm{BM}$ & MOM & REV & IVOL & CSK & CKT & RSK & $\mathrm{RKT}$ & MAX & ILLIQ \\
\hline I & $\begin{array}{l}-0.11 \\
(-0.25)\end{array}$ & $\begin{array}{l}0.30 \\
(2.32)\end{array}$ & $\begin{array}{l}-0.37 \\
(-4.40)\end{array}$ & $\begin{array}{l}-0.25 \\
(-0.93)\end{array}$ & $\begin{array}{c}0.01 \\
(1.03)\end{array}$ & & & & & & & & \\
\hline II & $\begin{array}{l}-0.09 \\
(-0.21)\end{array}$ & $\begin{array}{l}0.30 \\
(2.28)\end{array}$ & $\begin{array}{l}-0.38 \\
(-4.51)\end{array}$ & $\begin{array}{l}-0.23 \\
(-1.13)\end{array}$ & $\begin{array}{c}0.00 \\
(0.84)\end{array}$ & $\begin{array}{l}-0.02 \\
(-2.12)\end{array}$ & & & & & & & \\
\hline III & $\begin{array}{l}-0.07 \\
(-0.15)\end{array}$ & $\begin{array}{l}0.33 \\
(2.31)\end{array}$ & $\begin{array}{c}-0.39 \\
(-5.04)\end{array}$ & $\begin{array}{l}-0.26 \\
(-1.00)\end{array}$ & $\begin{array}{c}0.00 \\
(1.04)\end{array}$ & & $\begin{array}{l}-0.09 \\
(-0.92)\end{array}$ & & & & & & \\
\hline IV & $\begin{array}{l}-0.09 \\
(-0.21)\end{array}$ & $\begin{array}{l}0.30 \\
(2.26)\end{array}$ & $\begin{array}{l}-0.38 \\
(-4.51)\end{array}$ & $\begin{array}{l}-0.28 \\
(-1.09)\end{array}$ & $\begin{array}{c}0.00 \\
(1.02)\end{array}$ & & & $\begin{array}{l}-0.23 \\
(-0.43)\end{array}$ & & & & & \\
\hline V & $\begin{array}{l}-0.04 \\
(-0.10)\end{array}$ & $\begin{array}{l}0.29 \\
(2.30)\end{array}$ & $\begin{array}{c}-0.37 \\
(-4.72)\end{array}$ & $\begin{array}{l}-0.29 \\
(-1.12)\end{array}$ & $\begin{array}{c}0.00 \\
(0.99)\end{array}$ & & & & $\begin{array}{c}-0.04 \\
(-0.31)\end{array}$ & & & & \\
\hline VI & $\begin{array}{l}-0.08 \\
(-0.18)\end{array}$ & $\begin{array}{l}0.29 \\
(2.30)\end{array}$ & $\begin{array}{c}-0.37 \\
(-4.43)\end{array}$ & $\begin{array}{c}-0.26 \\
(-0.98)\end{array}$ & $\begin{array}{c}0.00 \\
(0.99)\end{array}$ & & & & & $\begin{array}{c}-0.59 \\
(-2.01)\end{array}$ & & & \\
\hline VII & $\begin{array}{l}-0.11 \\
(-0.25)\end{array}$ & $\begin{array}{l}0.28 \\
(2.27)\end{array}$ & $\begin{array}{c}-0.41 \\
(-4.63)\end{array}$ & $\begin{array}{l}-0.31 \\
(-1.24)\end{array}$ & $\begin{array}{c}0.01 \\
(1.16)\end{array}$ & & & & & & $\begin{array}{l}-0.06 \\
(-0.89)\end{array}$ & & \\
\hline VIII & $\begin{array}{l}-0.05 \\
(-0.12)\end{array}$ & $\begin{array}{l}0.36 \\
(2.56)\end{array}$ & $\begin{array}{l}-0.39 \\
(-4.87)\end{array}$ & $\begin{array}{l}-0.26 \\
(-1.00)\end{array}$ & $\begin{array}{c}0.00 \\
(1.02)\end{array}$ & & & & & & & $\begin{array}{l}-0.05 \\
(-1.61)\end{array}$ & \\
\hline IX & $\begin{array}{l}-0.11 \\
(-0.26)\end{array}$ & $\begin{array}{l}0.28 \\
(2.29)\end{array}$ & $\begin{array}{l}-0.44 \\
(-2.74)\end{array}$ & $\begin{array}{l}-0.23 \\
(-0.87)\end{array}$ & $\begin{array}{c}0.01 \\
(1.21)\end{array}$ & & & & & & & & $\begin{array}{c}-0.08 \\
(-0.60)\end{array}$ \\
\hline $\mathrm{X}$ & $\begin{array}{l}-0.01 \\
(-0.04)\end{array}$ & $\begin{array}{l}0.31 \\
(2.33)\end{array}$ & $\begin{array}{l}-0.50 \\
(-3.33)\end{array}$ & $\begin{array}{l}-0.29 \\
(-1.54)\end{array}$ & $\begin{array}{c}0.00 \\
(1.14)\end{array}$ & $\begin{array}{l}-0.02 \\
(-2.16)\end{array}$ & $\begin{array}{l}-0.22 \\
(-2.05)\end{array}$ & $\begin{array}{c}0.81 \\
(1.46)\end{array}$ & $\begin{array}{l}-0.12 \\
(-0.84)\end{array}$ & $\begin{array}{c}-0.17 \\
(-0.63)\end{array}$ & $\begin{array}{l}-0.05 \\
(-0.89)\end{array}$ & $\begin{array}{c}0.03 \\
(0.95)\end{array}$ & $\begin{array}{c}-0.10 \\
(-0.77)\end{array}$ \\
\hline XI & $\begin{array}{l}0.2 \\
(1.9\end{array}$ & & $\begin{array}{l}-0.47 \\
(-3.05)\end{array}$ & $\begin{array}{l}-0.28 \\
(-1.49)\end{array}$ & $\begin{array}{c}0.01 \\
(1.27)\end{array}$ & $\begin{array}{l}-0.02 \\
(-2.13)\end{array}$ & $\begin{array}{l}-0.22 \\
(-2.08)\end{array}$ & $\begin{array}{c}0.81 \\
(1.41)\end{array}$ & $\begin{array}{l}-0.17 \\
(-1.23)\end{array}$ & $\begin{array}{l}-0.17 \\
(-0.61)\end{array}$ & $\begin{array}{l}-0.02 \\
(-0.45)\end{array}$ & $\begin{array}{c}0.02 \\
(0.66)\end{array}$ & $\begin{array}{c}-0.08 \\
(-0.62)\end{array}$ \\
\hline
\end{tabular}


(A)

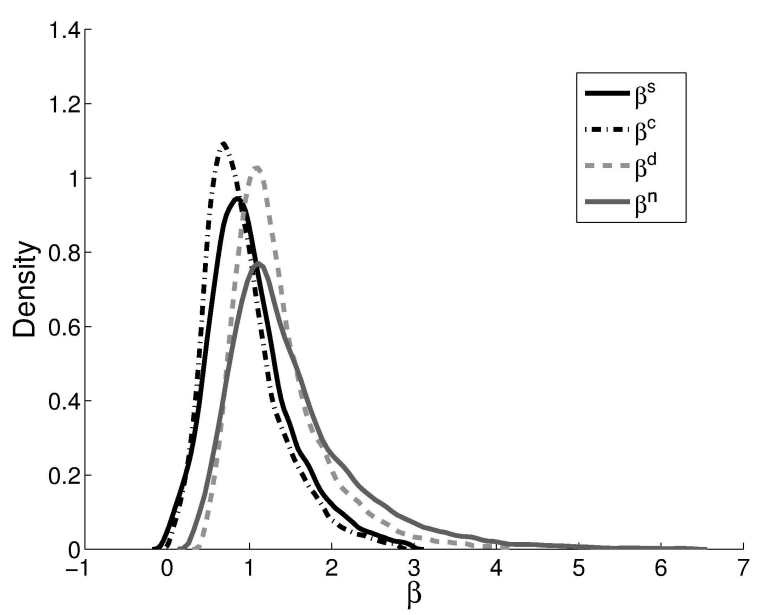

(B)

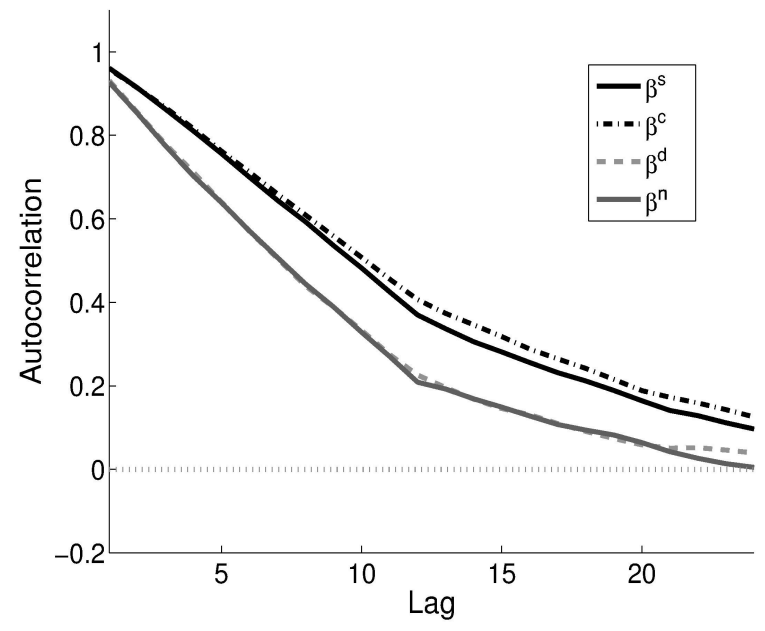

Figure 1: Distributions and autocorrelograms of betas

Panel A displays kernel density estimates of the unconditional distributions of the four different betas averaged across firms and time. Panel B shows the monthly autocorrelograms for the four different betas averaged across firms. 
(A) $\beta^{s}$
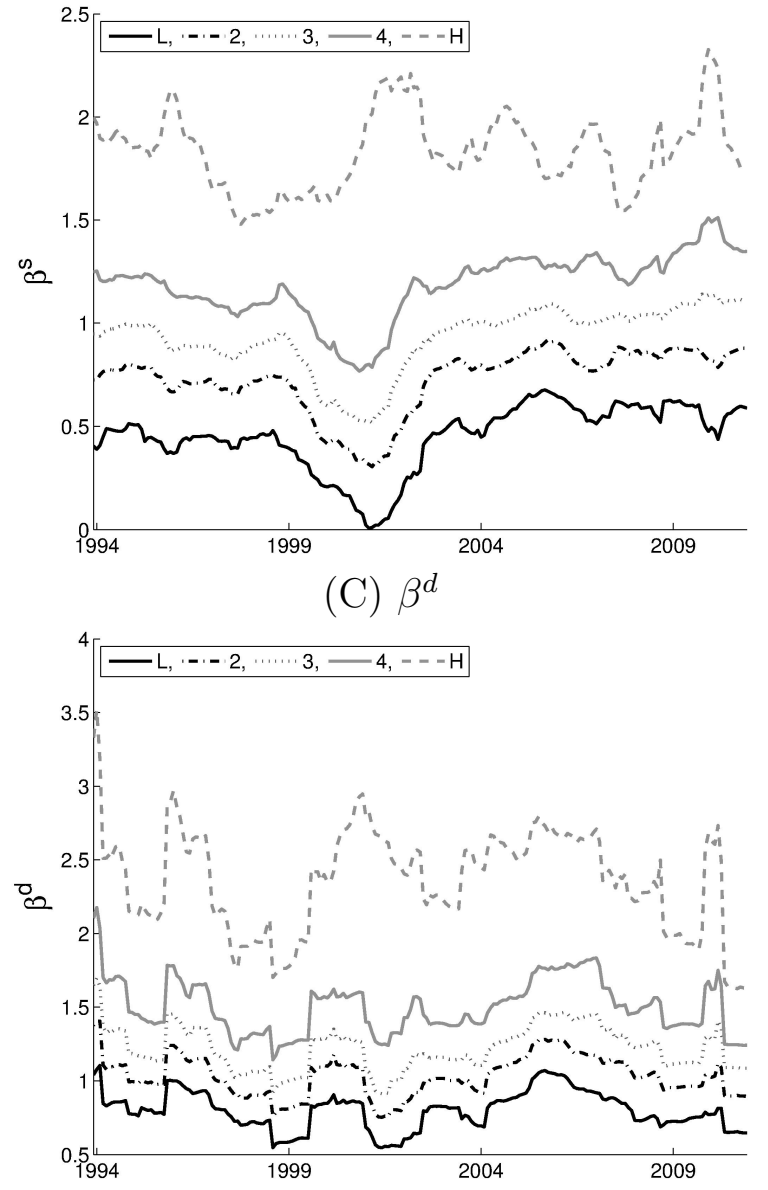

(B) $\beta^{c}$

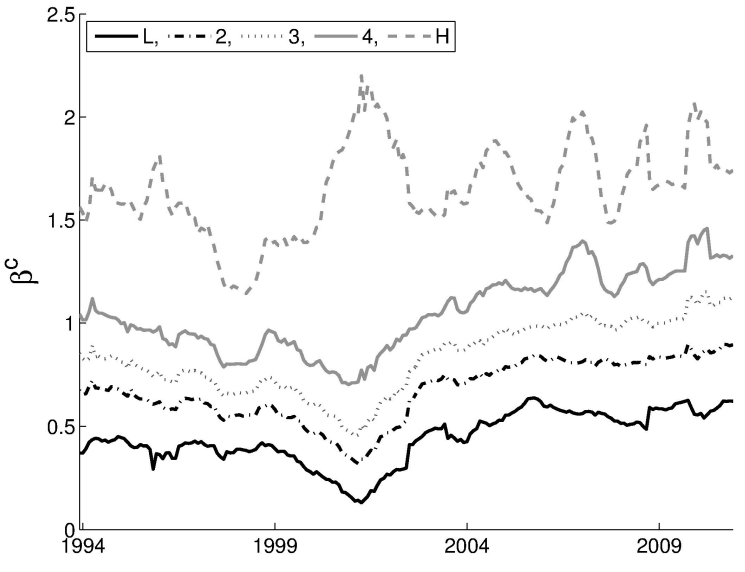

(D) $\beta^{n}$

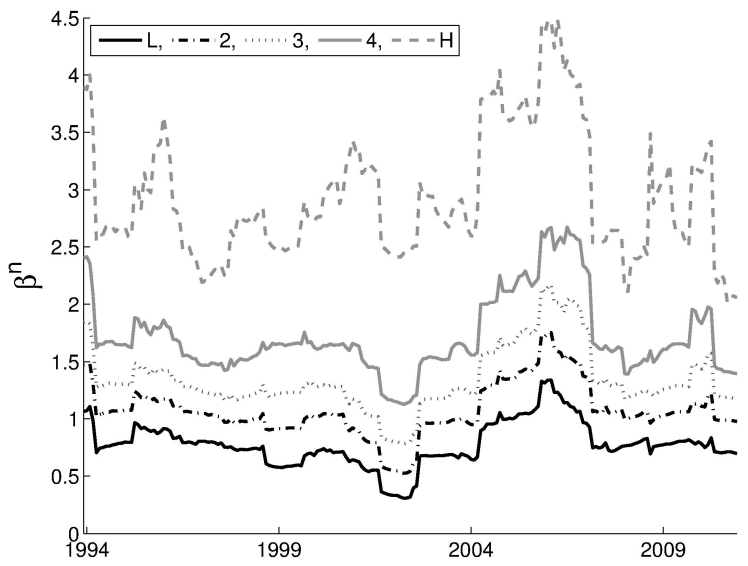

Figure 2: Time series plots of betas

The figure displays the times series of betas for equally weighted beta-sorted quintile portfolios. Panel A shows the results for the standard beta $\beta^{s}$-sorted portfolios, Panel B the continuous beta $\beta^{c}$-sorted portfolios, Panel $\mathrm{C}$ the discontinuous beta $\beta^{d}$-sorted portfolios, and Panel $\mathrm{D}$ the overnight beta $\beta^{n}$-sorted portfolios. 
(A)

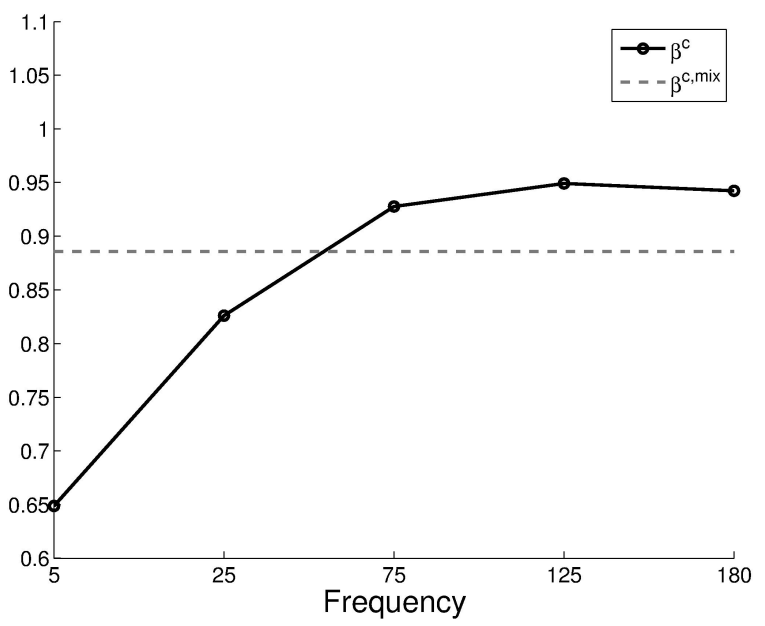

(B)

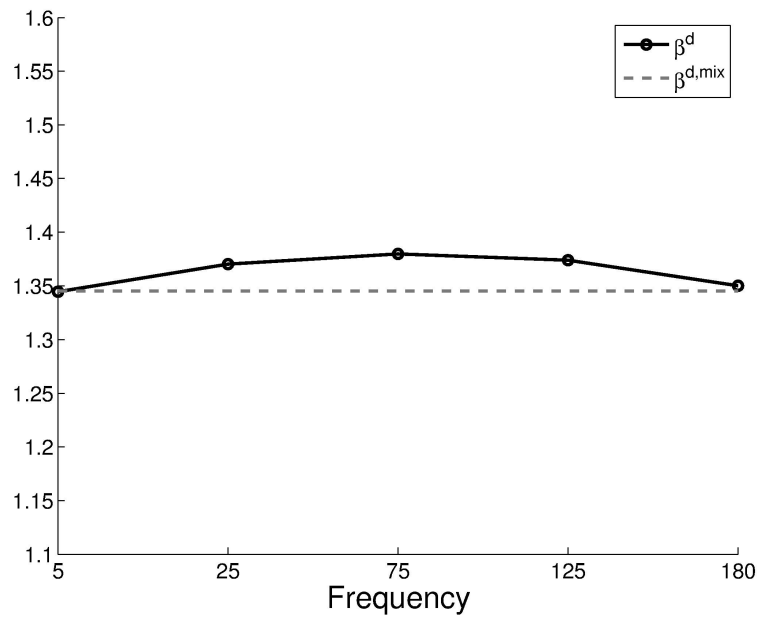

Figure 3: Signature plots for betas

Panel A shows the mean value of $\beta^{c}$ (solid line) averaged across stocks and time for different sampling frequencies (labeled in minutes on the x-axis). The dashed line gives the mean value of the mixed-frequency $\beta^{c}$. Panel B plots the same averaged estimates for $\beta^{d}$.

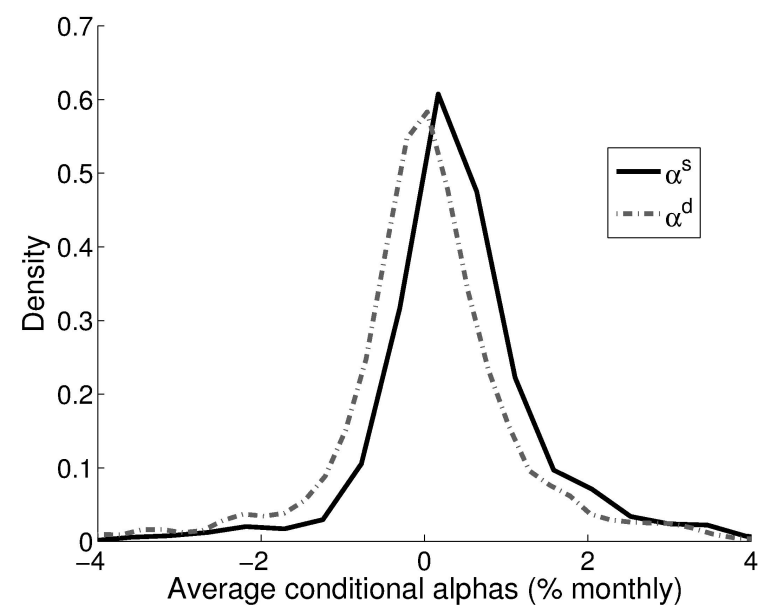

Figure 4: Average conditional alphas

The figure shows the cross-sectional distribution of the average conditional alphas based on the standard beta $\left(\alpha^{s}\right)$ and the continuous and two rough betas $\left(\alpha^{d}\right)$. 


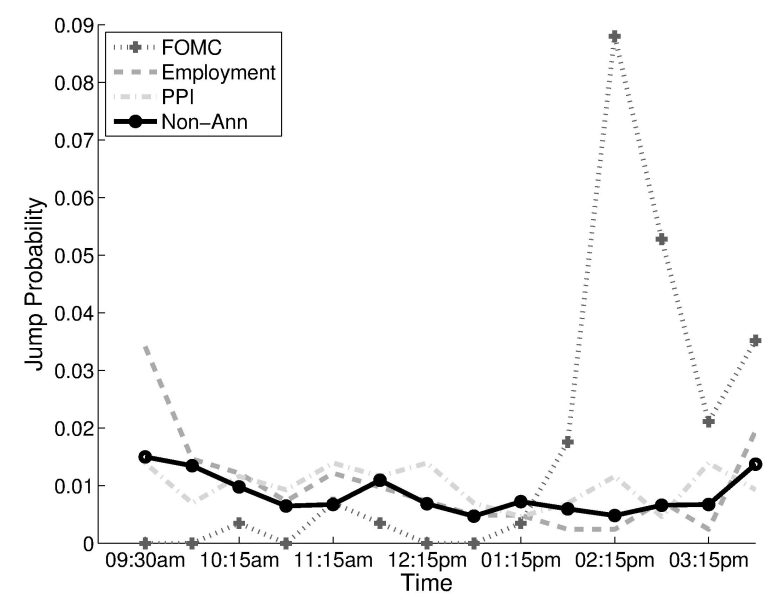

Figure 5: Jump intensity and macro announcement

The figure plots the average estimated jump intensity (probability) for the S\&P 500 market portfolio across regular trading hours on FOMC announcement days, Employment announcement days, PPI announcement days, and all other days (Non-Ann). 


\section{References}

Aït-Sahalia 2004. Disentangling Volatility from Jumps. Journal of Financial Economics, 74:487528.

Aït-Sahalia, Y., Cacho-Diaz, J., and Hurd, T. 2009. Portfolio Choice with Jumps: A Closed-Form Solution. Annals of Applied Probability, 19(2):556-584.

Amaya, D., Christoffersen, P., Jacobs, K., and Vasquez, A. 2013. Do Realized Skewness and Kurtosis Predict the Cross-Section of Equity Returns? Working paper, University of Quebec at Montreal, University of Toronto, University of Houston, and Instituto Tecnológico Autónomo de México.

Amihud, Y. 2002. Illiquidity and Stock Returns: Cross-Section and Time-Series Effects. Journal of Financial Markets, 5:31-56.

Andersen, T. G., Bollerslev, T., and Diebold, F. X. 2007a. Roughing It Up: Disentangling Continuous and Jump Components in Measuring, Modeling and Forecasting Asset Return Volatility. Review of Economics and Statistics, 89:701-720.

Andersen, T. G., Bollerslev, T., Diebold, F. X., and Labys, P. 2001. The Distribution of Realized Exchange Rate Volatility. Journal of the American Statistical Association, 42:42-55.

Andersen, T. G., Bollerslev, T., Diebold, F. X., and Vega, C. 2003. Micro Effects of Macro Annoucements: Real-Time Price Discovery in Foreign Exchange. American Economic Review, $93: 38-62$.

Andersen, T. G., Bollerslev, T., Diebold, F. X., and Vega, C. 2007b. Real-Time Price Discovery in Stock, Bond and Foreign Exchange Markets. Journal of International Economics, 73:251-277.

Andersen, T. G., Bollerslev, T., Diebold, F. X., and Wu, G. 2005. A Framework for Exploring the Macroeconomic Determinants of Systematic Risk. American Economic Review, 95:398-404.

Andersen, T. G., Bollerslev, T., Diebold, F. X., and Wu, G. 2006. Realized Beta: Persistence and Predictability. In Fomby, T. and Terrell, D., editors, Advances in Econometrics: Econometric Analysis of Economic and Financial Time Series, volume 20. Elsevier Science.

Andersen, T. G., Bollerslev, T., and Huang, X. 2011. A Reduced Form Framework for Modeling Volatility of Speculative Prices Based on Realized Variation Measures. Journal of Econometrics, 160:176-189.

Ang, A. and Chen, J. 2002. Asymmetric Correlations of Equity Portfolios. Journal of Financial Economics, 63:443-494.

Ang, A., Chen, J., and Xing, Y. 2006a. Downside Risk. Review of Financial Studies, 19:1191-1239.

Ang, A., Hodrick, R., Xing, Y., and Zhang, X. 2006b. The Cross-Section of Volatility and Expected Returns. Journal of Finance, 61:259-299.

Bali, T. G., Cakici, N., and Whitelaw, R. 2011. Maxing Out: Stocks as Lotteries and the CrossSection of Expected Returns. Journal of Financial Economics, 99:427-446. 
Bali, T. G., Engle, R. F., and Tang, Y. 2014. Dynamic Conditional Beta is Alive and Well in the Cross-Section of Daily Stock Returns. Working paper, Georgetown University, New York University, and Fordham University.

Bansal, R. and Yaron, A. 2004. Risks for the Long Run: A Potential Resolution of Asset Pricing Puzzles. Journal of Finance, 59:1481-1509.

Banz, R. W. 1981. The Relationship between Return and Market Value of Common Stock. Journal of Financial Economics, 9:3-18.

Barndorff-Nielsen, O. E., Hansen, P. R., Lunde, A., and Shephard, N. 2009. Realized Kernels in Practice: Trades and Quotes. Econometrics Journal, 12:C1-C32.

Barndorff-Nielsen, O. E. and Shephard, N. 2003. Realized Power Variation and Stochastic Volatility Models. Bernoulli, 9:243-265.

Barndorff-Nielsen, O. E. and Shephard, N. 2004a. Econometric Analysis of Realized Covariation: High Frequency Based Covariance, Regression, and Correlation in Financial Economics. Econometrica, 72:885-925.

Barndorff-Nielsen, O. E. and Shephard, N. 2004b. Power and Bipower Variation with Stochastic Volatility and Jumps. Journal of Financial Econometrics, 2:1-37.

Barndorff-Nielsen, O. E. and Shephard, N. 2006. Econometrics of Testing for Jumps in Financial Economics using Bipower Variation. Journal of Financial Econometrics, 4:1-30.

Barndorff-Nielsen, O. E. and Shephard, N. 2007. Variation, Jumps, Market Frictions and High Frequency Data in Financial Econometrics. In Blundell, R., Torsten, P., and Newey, W. K., editors, Advances in Economics and Econometrics: Theory and Applications. Econometric Society monographs, pages 328-372. Cambridge University Press, Cambridge.

Basu, S. 1977. Investment Performance of Common Stocks in Relation to Their Price-Earnings Ratios: A Test of the Efficient Market Hypothesis. Journal of Finance, 32:663-682.

Basu, S. 1983. The Relationship Between Earnings Yield, Market Value, and Return for NYSE Common Stocks: Further Evidence. Journal of Financial Economics, 12:129-156.

Berkman, H., Koch, P., Tuttle, L., and Zhang, Y. 2012. Paying Attention: Overnight Returns and the Hidden Cost of Buying at the Open. Journal of Financial and Quantitative Analysis, 47:715-741.

Bhandari, L. C. 1988. Debt/Equity Ratio and Expected Common Stock Returns: Empirical Evidence. Journal of Finance, 43:507-528.

Blume, M. 1970. Portfolio Theory: A Step Toward Its Practical Application. Journal of Business, $43: 152-173$.

Bollerslev, T., Engle, R., and Wooldridge, J. 1988. A Capital Asset Pricing Model with TimeVarying Covariances. Journal of Political Economy, 96:116-131.

Bollerslev, T., Law, T. H., and Tauchen, G. 2008. Risk, Jumps, and Diversification. Journal of Econometrics, 144:234-256. 
Bollerslev, T., Sizova, N., and Tauchen, G. 2012. Volatility in Equilibrium: Asymmetries and Dynamic Dependencies. Review of Finance, 16:31-80.

Bollerslev, T. and Todorov, V. 2011. Tails, Fears, and Risk Premia. Journal of Finance, 66:21652221.

Bollerslev, T., Todorov, V., and Li, S. Z. 2013. Jump Tails, Extreme Dependencies and the Distribution of Stock Returns. Journal of Econometrics, 172:307-324.

Bollerslev, T., Todorov, V., and Li, S. Z. 2014. Supplementary Appendix to: "Roughing up Beta: Continuous vs. Discontinuous Betas, and the Cross-Section of Expected Stock Returns". Working paper, Duke University, Northwestern University, and Michigan State University.

Bollerslev, T. and Zhang, B. Y. 2003. Measuring and Modeling Systematic Risk in Factor Pricing Models Using High-Frequency Data. Journal of Empirical Finance, 10:533-558.

Branch, B. and Ma, A. 2012. The Overnight Return: The Invisible Hand Behind the Intraday Return. Journal of Applied Finance, 22(2):90-100.

Campbell, J. and Cochrane, J. 1999. By Force of Habit: A Consumption Based Explanation of Aggregate Stock Market Behavior. Journal of Political Economy, 107:205-251.

Cliff, M., Cooper, M., and Gulen, H. 2008. Return Differences between Trading and Non-Trading Hours: Like Night and Day. Working paper, University of Utah, Analysis Group, and Purdue University.

Corradi, V., Distaso, W., and Fernandes, M. 2013. Conditional Alphas and Realized Betas. Working paper, University of Warwick, Imperial College London, and Queen Mary University.

Cremers, M., Halling, M., and Weinbaum, D. 2014. Aggregate Jump and Volatility Risk in the Cross-Section of Stock Returns. Journal of Finance, forthcoming.

Dimson, E. 1979. Risk Measurement when Shares are Subject to Infrequent Trading. Journal of Financial Economics, 7:197-226.

Duffie, D., Pan, J., and Singleton, K. 2000. Transform Analysis and Asset Pricing for Affine Jump-Diffusions. Econometrica, 68:1343-1376.

Eraker, B., Johannes, M., and Polson, N. 2003. The Impact of Jumps in Volatility and Returns. Journal of Finance, 58:1269-1300.

Fama, E. F., Fisher, L., Jensen, M., and Roll, R. 1969. The Adjustment of Stock Prices to New Information. International Economic Review, 10:1-21.

Fama, E. F. and French, K. R. 1992. The Cross-Section of Expected Stock Returns. Journal of Finance, 47:427-465.

Fama, E. F. and French, K. R. 1993. Common Risk Factors in the Returns on Stocks and Bonds. Journal of Financial Economics, 33:3-56.

Fama, E. F. and French, K. R. 2006. The Value Premium and the CAPM. Journal of Finance, 61:2163-2185. 
Fama, E. F. and MacBeth, J. D. 1973. Risk, Return, and Equilibrium: Empirical Tests. Journal of Political Economy, 81(3):607-636.

Ferson, W., Kandel, S., and Stambaugh, R. F. 1987. Tests of Asset Pricing with Time-Varying Expected Risk Premiums and Market Betas. Journal of Finance, 42:201-220.

Fleming, M. J. and Remolona, E. M. 1999. Price Formation and Liquidity in the U.S. Treasury Market: The Response to Public Information. Journal of Finance, 54:1901-1915.

Frazzini, A. and Pedersen, L. 2014. Betting Against Beta. Journal of Financial Economics, 111:125.

Fu, F. 2009. Idiosyncratic Risk and the Cross-Section of Expected Stock Returns. Journal of Financial Economics, 91:24-37.

Gabaix, X. 2012. Variable Rare Disasters: An Exactly Solved Framework for Ten Puzzles in Macrofinance. Quarterly Journal of Economics, 127:645-700.

Han, B. and Kumar, A. 2013. Speculative Retail Trading and Asset Prices. Journal of Financial and Quantitative Analysis, 48:377-404.

Hansen, P. and Lunde, A. 2006. Realized Variance and Market Microstructure Noise. Journal of Business and Economic Statistics, 24:127-161.

Harvey, C. 1989. Time-varying Conditional Covariances in Tests of Asset Pricing Models. Journal of Financial Economics, 24:289-317.

Harvey, C. and Siddique, A. 2000. Conditional Skewness in Asset Pricing Tests. Journal of Finance, 55:1263-1295.

Hedegaard, E. and Hodrick, R. J. 2013. Estimating the Conditional CAPM with Overlapping Data Inference. Working paper, Arizona State University and Columbia University.

Jacod, J. and Protter, P. 2012. Discretization of Processes. Springer-Verlag, Berlin.

Jacod, J. and Shiryaev, A. 2002. Limit Theorems for Stochastic Processes. Springer-Verlag, Berlin.

Jagannathan, R. and Wang, Z. 1996. The Conditional CAPM and the Cross-Section of Expected Stock Returns. Journal of Finance, 51:3-53.

Jegadeesh, N. 1990. Evidence of Predictable Behavior of Security Returns. Journal of Finance, 45:881-898.

Jegadeesh, N. and Titman, S. 1993. Returns to Buying Winners and Selling Losers: Implications for Stock Market Efficiency. Journal of Finance, 48:65-92.

Jiang, G. and Yao, T. 2013. Stock Price Jumps and Cross-Sectional Return Predictability. Journal of Financial and Quantitative Analysis, 48(5):1519-1544.

Lahaye, J., Laurent, S., and Neely, C. 2011. Jumps, Cojumps and Macro Annoucements. Journal of Applied Econometrics, 26:893-921.

Lee, S. 2012. Jumps and Information Flow in Financial Markets. Review of Financial Studies, $25(2): 439-479$. 
Lehmann, B. N. 1990. Fads, Martingales, and Market Efficiency. Quarterly Journal of Economics, 105:1-28.

Lettau, M. and Ludvigson, S. 2001. Resurrecting the (C)CAPM: a Cross-Sectional Test when Risk Premia are Time-Varying. Journal of Political Economy, 109:1238-1287.

Lewellen, J. and Nagel, S. 2006. The Conditional CAPM Does Not Explain Asset-Price Anomalies. Journal of Financial Economics, 82:289-314.

Liu, J., Longstaff, F., and Pan, J. 2003a. Dynamic Asset Allocation with Event Risk. Journal of Finance, 58:231-259.

Liu, J., Longstaff, F., and Pan, J. 2003b. Dynamic Derivative Strategies. Journal of Financial Economics, 69:401-430.

Longstaff, F. 1989. Temporal Aggregation and the Continuous-Time Capital Asset Pricing Model. Journal of Finance, 44:871-887.

Lucca, D. and Moench, E. 2014. The Pre-FOMC Announcement Drift. Journal of Finance, forthcoming.

Merton, R. C. 1973. An Intertemporal Capital Asset Pricing Model. Econometrica, 41:867-887.

Merton, R. C. 1976. Option Pricing when Underlying Asset Returns are Discontinuous. Journal of Financial Economics, 3:125-144.

Pan, J. 2002. The Jump Risk Premium Implicit in Options: Evidence from an Integrated TimeSeries Study. Journal of Financial Economics, 53:3-50.

Patton, A. and Verardo, M. 2012. Does Beta Move with News? Firm-Specific Information Flows and Learning About Profitability. Review of Financial Studies, 25:2789-2839.

Roll, R. 1977. A Critique of the Asset Pricing Theory's Tests - Part I: On Past and Potential Testability of the Theory. Journal of Financial Economics, 4:129-176.

Rosenberg, B., Reid, K., and Lanstein, R. 1985. Persuasive Evidence of Market Inefficiency. Journal of Portfolio Management, 11:9-16.

Savor, P. and Wilson, M. 2014. Asset Pricing: A Tale of Two Days. Journal of Financial Economics, 113:171-201.

Scholes, M. and Williams, J. 1977. Estimating Betas from Non-Synchronous Data. Journal of Financial Economics, 5:308-328.

Shanken, J. 1992. On the Estimation of Beta-Pricing Models. Review of Financial Studies, 5:1-55.

Sheppard, K. 2006. Realized Covariance and Scrambling. Working paper, University of Oxford.

Stambaugh, R. F., Yu, J., and Yuan, Y. 2014. Arbitrage Asymmetry and the Idionsyncratic Volatility Puzzle. Journal of Finance, forthcoming.

Stattman, D. 1983. Book Values and Stock Returns. The Chicago MBA: A Journal of Selected Papers, 4:25-45. 
Todorov, V. and Bollerslev, T. 2010. Jumps and Betas: A New Framework for Disentangling and Estimating Systematic Risks. Journal of Econometrics, 157:220-235.

Wachter, J. A. 2013. Can Time-Varying Risk of Rare Disasters Explain Aggregate Stock Market Volatility? Journal of Finance, 68:987-1035.

Yan, S. 2011. Jump Risk, Stock Returns, and Slope of Implied Volatility Smile. Journal of Financial Economics, 99:216-233. 
2014-31: $\quad$ Peter Christoffersen, Asger Lunde and Kasper V. Olesen: Factor Structure in Commodity Futures Return and Volatility

2014-32: $\quad$ Ulrich Hounyo: The wild tapered block bootstrap

2014-33: Massimiliano Caporin, Luca Corazzini and Michele Costola: Measuring the Behavioral Component of Financial Fluctuations: An Analysis Based on the S\&P 500

2014-34: $\quad$ Morten Ørregaard Nielsen: Asymptotics for the conditional-sum-of-squares estimator in multivariate fractional time series models

2014-35: Ulrich Hounyo: Bootstrapping integrated covariance matrix estimators in noisy jump-diffusion models with non-synchronous trading

2014-36: Mehmet Caner and Anders Bredahl Kock: Asymptotically Honest Confidence Regions for High Dimensional

2014-37: $\quad$ Gustavo Fruet Dias and George Kapetanios: Forecasting Medium and Large Datasets with Vector Autoregressive Moving Average (VARMA) Models

2014-38: Søren Johansen: Times Series: Cointegration

2014-39: $\quad$ Søren Johansen and Bent Nielsen: Outlier detection algorithms for least squares time series regression

2014-40: $\quad$ Søren Johansen and Lukasz Gatarek: Optimal hedging with the cointegrated vector autoregressive model

2014-41: $\quad$ Laurent Callot and Johannes Tang Kristensen: Vector Autoregressions with Parsimoniously Time Varying Parameters and an Application to Monetary Policy

2014-42: $\quad$ Laurent A. F. Callot, Anders B. Kock and Marcelo C. Medeiros: Estimation and Forecasting of Large Realized Covariance Matrices and Portfolio Choice

2014-43: $\quad$ Paolo Santucci de Magistris and Federico Carlini: On the identification of fractionally cointegrated VAR models with the $F(d)$ condition

2014-44: $\quad$ Laurent Callot, Niels Haldrup and Malene Kallestrup Lamb: Deterministic and stochastic trends in the Lee-Carter mortality model

2014-45: $\quad$ Nektarios Aslanidis, Charlotte Christiansen, Neophytos Lambertides and Christos S. Savva: Idiosyncratic Volatility Puzzle: Influuence of Macro-Finance Factors

2014-46: Alessandro Giovannelli and Tommaso Proietti: On the Selection of Common Factors for Macroeconomic Forecasting

2014-47: $\quad$ Martin M. Andreasen and Andrew Meldrum: Dynamic term structure models: The best way to enforce the zero lower bound

2014-48: $\quad$ Tim Bollerslev, Sophia Zhengzi Li and Viktor Todorov: Roughing up Beta: Continuous vs. Discontinuous Betas, and the Cross-Section of Expected Stock Returns 